\title{
Modelos baseados em agentes aplicados à dinâmica de preços do mercado imobiliário
}

\author{
Manuella de Oliveira Antunes
}

DISSERTAÇÃO APRESENTADA

AO

Instituto de MATEMÁtiCA E EstatísticA

DA

Universidade de São PaUlo

PARA

OBTENÇÃO DO TÍTULO

DE

MESTRE EM CIÊNCIAS

\begin{abstract}
Programa: Estatística
Trabalho patrocinado pelo

Programa de Pós-Graduação do

Banco Central do Brasil
\end{abstract}

Orientador: Prof. Dr. Fernando Pigeard de Almeida Prado

São Paulo, março de 2016 


\title{
Modelos baseados em agentes aplicados à dinâmica de preços do mercado imobiliário
}

\author{
Esta versão da dissertação contém as correções e alterações sugeridas \\ pela Comissão Julgadora durante a defesa da versão original do trabalho, \\ realizada em 15/04/2016. Uma cópia da versão original está disponível no \\ Instituto de Matemática e Estatística da Universidade de São Paulo.
}

Comissão Julgadora:

- Prof. Dr. Fernando Pigeard de Almeida Prado (orientador) - FFCLRP-USP

- Prof. Dr. Vladimir Belitsky - IME-USP

- Prof. Dr. Alex Luiz Ferreira - FEA-RP/USP 
Ao Flavinho, o gigante sobre cujos ombros eu me apoio. 


\section{Agradecimentos}

Quando eu comecei a trabalhar, fiquei três anos sem estudar as coisas de que sempre gostei. Então, quando eu decidi que pediria o afastamento para estudar essas coisas de novo, percebi que tinha esquecido quase tudo! Eu relembrei muitas coisas e aprendi muitas coisas novas nesses 2,2 anos de estudo, e o mais interessante: consegui juntar tudo e usar num problema do trabalho. E tenho a agradecer a muitas pessoas que, com todo o apoio, amizade e ensinamentos, me ajudaram a fazer este trabalho.

Em primeiro lugar, agradeço ao Flavinho, meu maior apoiador. Agradeço também ao Banco Central pelo apoio financeiro e ao IME por ter me aceitado no mestrado; ao meu orientador, Professor Fernando, que aceitou me orientar num trabalho aplicado e me ajudou durante todo o trabalho e na defesa; aos membros da banca e, em especial, ao Professor Vladimir, que me ajudou também na escolha das matérias; e aos funcionários do IME, em especial à Regiane, que me ajudou no processo da defesa.

Agradeço também àqueles que me ajudaram a entrar no mestrado, e àqueles que me ajudaram durante esse período: ao Rafael Ando, que se lembrou de mim na porta do laboratório, quando todos já tinham se esquecido; ao Romis, ao Paiva e ao Fábio Lacerda, por confiarem em mim; ao Fernando Chertman, pelas dicas de ex-aluno; à Letícia e à Gabi, pela amizade e pelo apoio; ao Leonardo, por ter me oferecido uma aposta de hedge quando eu achei que não passaria; à Nancy, que, mesmo (secretamente) não querendo que o afastamento saísse, me ajudou no processo; à Regina, por ser minha mãe adotiva; à Cissa, ao Guilherme, ao Victor, ao Helder, à Gisele, à Yan Fei e ao André pela ajuda nos cursos e pela amizade; à Mamãe, que veio prontamente me socorrer no meio do afastamento; ao Moltocaro, por ter me deixado usar uma mesa da sua equipe quando eu estava precisando, e aos colegas da Dicre por terem me acolhido na equipe; aos novos colegas da Dimec, que torceram por mim no dia da defesa, e em especial à Mari e ao Michel, que se sujeitaram a uma avant première ininteligível.

Finalmente, agradeço àqueles sem cujos ensinamentos eu não teria conseguido entrar no mestrado no IME nem fazer a dissertação: ao Pancho pela teoria de conjuntos; ao Décio pelos números negativos e equações algébricas; ao Fausto pela fatoração de polinômios; à Jane 
pelas equações de segundo grau; à Tia Miriam pelos logarítmos, pelas exponenciais, funções compostas e inequações de segundo grau; ao Fausto (de novo) pelas funções trigonométricas; ao Marcão pelas matrizes e determinantes, probabilidades e combinatória; ao Paulo Wanner pelos números complexos; ao Herbert pela geometria analítica; ao Gilli pelos limites, pela regra de L'Hôpital, pelas derivadas e integrais; ao Ary pela álgebra linear; à Ketty e ao Yaro pelas equações diferenciais e transformadas de Laplace; ao Maurice a ao Denis por mais equações diferenciais; ao Violaro pelas séries e funções analíticas; ao Amaury pelos sistemas discretos; ao Geromel pela análise linear de sistemas e por nos proibir de usar calculadora; ao Paulo Valente pela otimização não-linear; ao Bordin pela análise real; ao Hoffmann pelas regressões e análise de variância; ao João Marcos e ao Renato pela teoria de probabilidades, processos estocásticos e inferência; ao Bruno e à Iara por mais análise combinatória e teoria de probabilidades; ao Luis Gustavo por muito mais teoria de probabilidades e inferência; à Gisela pela análise de sobrevivência; e ao Gilberto pelos MLGs. 


\section{Resumo}

Um dos aspectos regulatórios fundamentais para o mercado imobiliário no Brasil são os limites para obtenção de financiamento no Sistema Financeiro de Habitação. Esses limites podem ser definidos de forma a aumentar ou reduzir a oferta de crédito neste mercado, alterando o comportamento dos seus agentes e, com isso, o preço de mercado dos imóveis. Neste trabalho, propomos um modelo de formação de preços no mercado imobiliário brasileiro com base no comportamento dos agentes que o compõem. Os agentes vendedores têm comportamento heterogêneo e são influenciados pela demanda histórica, enquanto que os agentes compradores têm o seu comportamento determinado pela disponibilidade de crédito. Esta disponibilidade de crédito, por sua vez, é definida pelos limites para concessão de financiamento no Sistema Financeiro de Habitação. Verificamos que o processo markoviano que descreve preço de mercado converge para um sistema dinâmico determinístico quando o número de agentes aumenta, e analisamos o comportamento deste sistema dinâmico. Mostramos qual é a família de variáveis aleatórias que representa o comportamento dos agentes vendedores de forma que o sistema apresente um preço de equilíbrio não trivial, condizente com a realidade. Verificamos ainda que o preço de equilíbrio depende não só das regras de concessão de financiamento no Sistema Financeiro de Habitação, como também do preço de reserva dos compradores e da memória e da sensibilidade dos vendedores a alterações na demanda. A memória e a sensibilidade dos vendedores podem levar a oscilações de preços acima ou abaixo do preço de equilíbrio (típicas de processos de formação de bolhas); ou até mesmo a uma bifurcação de Neimark-Sacker, quando o sistema apresenta dinâmica oscilatória estável.

Palavras-chave: mercado imobiliário, Sistema Financeiro de Habitação, agentes heterogêneos, processos markovianos, sistemas dinâmicos não-lineares, bifurcação de NeimarkSacker. 


\section{Abstract}

One of the fundamental regulatory aspects for the housing market in Brazil are the limits for obtaining a residential mortgage loan within the Sistema Financeiro de Habitação. These limits can be defined so as to increase or reduce credit supply in this market, changing its agents' behavior and, therefore, the housing market price. In this work we propose a pricing model for the brazilian housing market based on the behavior of its agents. Sellers have heterogeneous behavior and are influenced by the historical demand, while buyers' behavior is determined by credit availability. The availability of credit is, in its turn, defined by the regulatory limits for obtaining a residential mortgage loan. We have verified that the Markov process which describes the market price converges to a deterministic dynamical system as the number of agents increase, and we have analysed the behavior of this emerging system. We show which family of random variables may represent the behavior of sellers so that the system has a nontrivial equilibrium price, consistent with reality. We have also verified that the equilibrium price depends not only on the regulatory limits for obtaing a loan, but also on buyers' reserve price and on sellers' memory and sensitivity to changes in the demand. Sellers' memory and sensitivity to changes in the demand can result in price oscillations above or below the equilibrium level, which is typical in bubble formation processes; or even in a Neimark-Sacker bifurcation, when the price has a stable oscillatory dynamics.

Keywords: housing market, regulatory limits on mortgage loans, heterogeneous agents, Markov processes, nonlinear dynamic systems, Neimark-Sacker bifurcation. 


\section{Sumário}

1 Introdução 1

1.1 Modelos de agentes e o mercado imobiliário . . . . . . . . . . . . 1

1.1.1 Funcionamento do mercado fora do Brasil . . . . . . . . . . . . 3

1.1.2 Funcionamento do mercado no Brasil . . . . . . . . . . . . 5

1.2 O financiamento imobiliário no Brasil . . . . . . . . . . . . . 6

1.2.1 Direcionamento obrigatório da poupança .......... 6

1.2.2 O Sistema Financeiro de Habitação . . . . . . . . . . . . . . . 7

1.2.3 Fundo de Garantia do Tempo de Serviço (FGTS) . . . . . . . . . . 9

1.2.4 Carteira hipotecária e outros limites . . . . . . . . . . 10

1.3 Justificativa . . . . . . . . . . . . . . . . . . . 11

2 Definição do modelo $\quad 13$

2.1 Definição do mercado . . . . . . . . . . . . . . . . . . . . . . 13

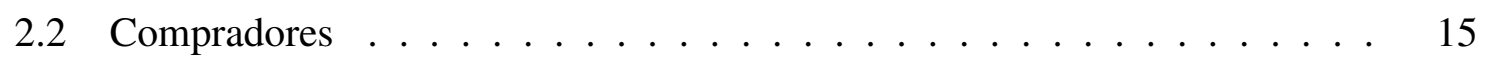

2.2.1 Condições para obter os recursos para compra . . . . . . . . . . 16

2.2.2 Compradores potenciais . . . . . . . . . . . . . 19

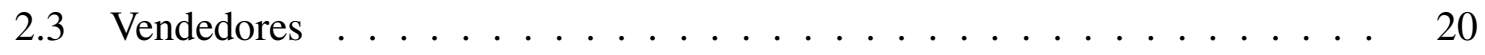

2.3.1 Distribuição do valor dos anúncios . . . . . . . . . . . . . 21

2.3.2 Vendedores potenciais ................... 22 
2.4 Função de demanda relativa $\Delta_{t} \ldots \ldots \ldots \ldots \ldots$. . . . . . . . 24

2.4.1 Rigidez dos preços na tendência de queda . . . . . . . . . . . 25

2.5 Número e valor das transações . . . . . . . . . . . . . . . . . 26

2.5.1 Número de transações . . . . . . . . . . . . . . . . . . 26

2.5.2 Valor das transações $\ldots \ldots . \ldots . \ldots . . \ldots 28$

3 Análise do modelo 33

3.1 Descrição resumida do modelo . . . . . . . . . . . . . . 33

3.2 Convergência em número de agentes . . . . . . . . . . . . . . . . . 34

3.2.1 Distribuição da função de demanda relativa . . . . . . . . . . . . 35

3.2.2 Preço de mercado . . . . . . . . . . . . . . . . . 37

3.3 Dinâmica dos preços . . . . . . . . . . . . . . . . . . 39

3.3.1 Tendência do preço de mercado . . . . . . . . . . . . . 40

3.3.2 Preço de equilíbrio .................... 46

3.3.3 Autovalores no ponto de equilíbrio . . . . . . . . . . . . . 49

3.3.4 Vendedores e compradores potenciais no equilíbrio . . . . . . . 52

4 Simulação $\quad 53$

4.1 Distribuição de renda e riqueza . . . . . . . . . . . . . . 53

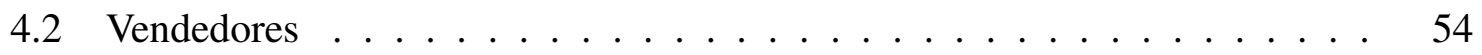

4.3 Estabilidade de preços quando $\delta^{\star}=1 \ldots \ldots \ldots$

4.4 Caso geral: estabilidade dos preços quando $\delta^{\star} \neq 1 \ldots \ldots$

4.5 Memória dos vendedores e sensibilidade à demanda . . . . . . . . . . . . . 58

4.6 Efeito de uma mudança nas regras de financiamento . . . . . . . . . . . 61

4.7 Comparação entre a aproximação $\left(p_{t}, \delta_{t}\right)$ e a realização de $\left(\boldsymbol{P}_{t}, \Delta_{t}\right) \ldots \ldots 1$

4.8 Bifurcação de Neimark-Sacker . . . . . . . . . . . . . . . 62

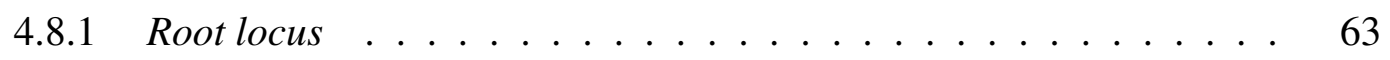

4.8.2 Dinâmica do preço e retrato de fase . . . . . . . . . . . 63 
$\begin{array}{ll}\text { A Poupança } & 73\end{array}$

A.1 Taxa de remuneração . . . . . . . . . . . . . . . . . . 73

A.2 Encaixe compulsório e direcionamento obrigatório . . . . . . . . . 74

B Demonstrações $\quad 77$

B.1 Demonstração do Fato $13 \ldots$. . . . . . . . . . . . . . . . . . . 77

B.2 Demonstração da Proposição 15 . . . . . . . . . . . . . . . . . 78

B.3 Demonstração do Lema $18 \ldots \ldots$. . . . . . . . . . . . . 79

B.4 Demonstração do Lema 20 . . . . . . . . . . . . . . . . . . . . . . . . 80

B.5 Demonstração do Teorema $21 \ldots \ldots$. . . . . . . . . . 80

B.6 Demonstração do Lema 22 . . . . . . . . . . . . . . . . . . . . . . . . 81

B.7 Demonstração do Lema $23 \ldots \ldots$. . . . . . . . . . . . . 82

B.8 Demonstração da Proposição 26 . . . . . . . . . . . . . . . . . . . . 84

B.9 Demonstração da Proposição $27 \ldots \ldots$. . . . . . . . . . . . . 85

B.10 Demonstração da Proposição 31 . . . . . . . . . . . . . . . 87

B.11 Demonstração da Proposição 34 . . . . . . . . . . . . . . . . . . . . . 88

B.12 Demonstração da Proposição 35 . . . . . . . . . . . . . . . . . . . . 90

B.13 Demonstração da Proposição 36 . . . . . . . . . . . . . . . . . . . . . . 92 


\section{Nomenclatura}

$\alpha \quad$ parâmetro de memória dos vendedores

$\boldsymbol{a}^{(i)} \quad$ variável com distribuição $\Psi$, que representa a pressa dos vendedores

$\boldsymbol{A}_{t}^{(i)} \quad$ valor do anúncio (ask) feito pelo $i$-ésimo vendedor no instante $t$

$\boldsymbol{B}_{t}^{(j)} \quad$ valor do bid do $i$-ésimo comprador no instante $t$

$\boldsymbol{I}_{t}^{(i)} \quad$ variável indicadora se o $i$-ésimo vendedor é vendedor potencial

$\boldsymbol{J}_{t}^{(j)} \quad$ variável indicadora se o $j$-ésimo comprador obtém os recursos para entrar no mercado

$\mathbf{N}_{t}^{(A)} \quad$ número de vendedores potenciais no instante $t$

$\mathbf{N}_{t}^{(B)} \quad$ número de compradores potenciais no instante $t$

$\boldsymbol{n}_{t} \quad$ número de transações ocorridas no instante $t$

$\boldsymbol{P}_{t} \quad$ preço de mercado no instante $t$

$\boldsymbol{T}_{t}^{(k)} \quad$ valor da $k$-ésima transação fechada no instante $t$

$\boldsymbol{W}^{(i)} \quad$ riqueza do $i$-ésimo comprador

$\boldsymbol{Y}^{(i)} \quad$ renda do $i$-ésimo comprador

$\Delta_{t} \quad$ função de demanda relativa

$\delta_{t} \quad$ valor determinístico para o qual converge $\Delta_{t}$

$\lambda \quad$ parâmetro de sensibilidade dos vendedores à demanda

$\mathfrak{g}\left(\boldsymbol{P}_{t-1} ; b, k, R\right)$ probabilidade de um comprador obter os recursos para entrar no mercado no instante $t$

$\mathfrak{h}_{\Psi, b}\left(\Delta_{t}\right) \quad$ função que relaciona o preço no instante atual com o preço no instante anterior 
$\Psi$

$\psi(x)$

$\rho$

$\tau$

b

C

$$
f_{Y, W}(y, w)
$$

$p_{t}$

$P_{\max }$

$R$

$r$

$S$

V

BCB

CEF

CLTV

CLT

CMN

FGTS

IF

IR

Razão entre o número de vendedores e o número de compradores

distribuição da pressa dos vendedores

densidade de $\boldsymbol{a}^{(i)}$

limite máximo da fração de comprometimento de renda

prazo máximo de financiamento em meses

parâmetro do preço de reserva dos compradores

número de agentes compradores

densidade conjunta de renda e riqueza

valor determinístico para o qual converge $\boldsymbol{P}_{t}$

limite máximo do valor do imóvel financiado

limite máximo de LTV

taxa de juros mensal do financiamento no âmbito de SFH

estoque de imóveis

número de agentes vendedores

Banco Central do Brasil

Caixa Econômica Federal

current loan to value

Consolidação das Leis do Trabalho

Conselho Monetário Nacional

Fundo de Garantia por Tempo de Serviço

instituição financeira

Imposto sobre a renda e proventos de qualquer natureza

Imposto sobre transmissão de bens imóveis

loan to value

Sistema de Amortizações Constantes 
SBPE

SFH

SFI

SFN

TR

VA

Freddie Mac
Sistema Brasileiro de Poupança e Empréstimo

Sistema Financeiro de Habitação

Sistema Financeiro Imobiliário

Sistema Financeiro Nacional

taxa referencial

variável aleatória

Federal Home Loan Mortgage Corporation 


\section{Lista de Figuras}

2.2.1 Região de integração para obter $\mathbb{P}\left(\boldsymbol{J}_{t}^{(j)}=1\right) \ldots \ldots \ldots \ldots \ldots$

2.3.1 Densidade hipotética de $\boldsymbol{a}^{(i)} \ldots \ldots \ldots \ldots \ldots \ldots$

2.3.2 Densidade hipotética de $\boldsymbol{A}_{t}^{(i)}$ quando a liquidez aumenta e diminui. . . . . . 22

2.5.1 Valores das transações, $\boldsymbol{T}_{t}^{(k)} \ldots \ldots \ldots \ldots \ldots$

2.5.2 Distribuição de $\boldsymbol{A}_{t}^{(i)}$ e de $\boldsymbol{T}_{t}^{(k)} \ldots \ldots \ldots \ldots \ldots \ldots \ldots$

3.3.1 Condição para haver tendência de aumento do preço. . . . . . . . . . . . . 41

3.3.2 A função $\mathfrak{h}_{\Psi, b}(\delta)$, quando $b>1$ e $\lim _{x \rightarrow 0} \frac{x \psi(x)}{\Psi(x)}=c>\frac{1}{b-1} \ldots \ldots \ldots \ldots$

3.3.3 Gráfico da função $\mathfrak{h}_{\Psi, b}(\delta)$ para $\Psi(x)=\left[1+\left(\frac{x}{\beta}\right)^{-\alpha}\right]^{-\gamma}$ e vários valores de $b .46$

4.3.1 Simulação do modelo para os parâmetros da Tabela 4.1 . . . . . . . . . . 56

4.4.1 Simulação do modelo para os parâmetros da Tabela 4.2 . . . . . . . . . . 59

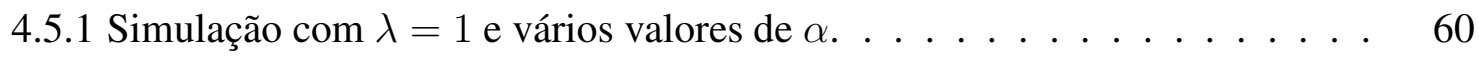

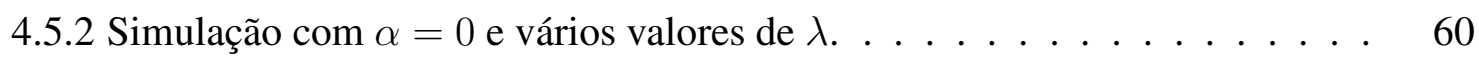

4.6.1 Efeito de uma flexibilização nas condições para obtenção de financiamento sobre o preço de mercado. . . . . . . . . . . . . . . . . 61

4.7.1 Comparação entre $\left(\boldsymbol{P}_{t}, \Delta_{t}\right)$ e $\left(p_{t}, \delta_{t}\right) \ldots \ldots \ldots \ldots \ldots$. . . . . . 62

4.8.1 Root locus dos autovalores para $\alpha \in[0,1]$ e vários valores de $\lambda . \quad \ldots . . .63$

4.8.2 Dinâmica do preço quando ocorre a bifurcação. . . . . . . . . . . . . . 65

4.8.3 Retrato de fase quando ocorre a bifurcação. . . . . . . . . . . . . . 66 
4.8.4 Órbita estável com $\alpha=0,4$ e $\lambda=1,3 \ldots \ldots \ldots 66$

A.1.1 Taxa Selic, TR e remuneração da poupança. . . . . . . . . . . . . . . . . . 74

B.12. Gráfico da função $f(\Lambda)=\Lambda^{2}-4 \Lambda-4 \Xi$, para $\Xi>0$ fixo. . . . . . . . 91 


\section{Lista de Tabelas}

1.1 Percentual do volume de financiamento imobiliário no SFN na data-base

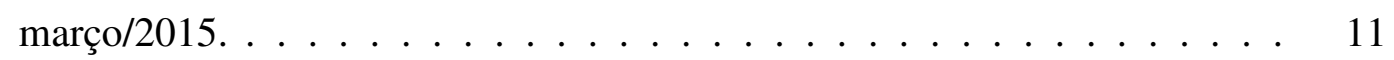

4.1 Parâmetros da simulação para equilíbrio com $\delta^{\star}=1 \ldots \ldots$. . . . . . . 56

4.2 Parâmetros da simulação para equilíbrio com $\delta^{\star} \neq 1 \ldots \ldots$. . . . . . 58

4.3 Parâmetros da simulação para valores diferentes de memória de sensibilidade à demanda. . . . . . . . . . . . . . . . . . . . . 5 59 


\section{Capítulo 1}

\section{Introdução}

\subsection{Modelos de agentes e o mercado imobiliário}

Os modelos de apreçamento de ativos financeiros normalmente se baseiam no princípio de não-arbitragem, na hipótese de eficiência dos mercados, ou usam equivalência de fluxos esperados para avaliar o valor do ativo. Ocorre que, no mercado imobiliário, onde os agentes são predominantemente indivíduos negociando as casas onde moram, essas hipóteses não são atendidas $[1,2,3]$, por diversos motivos que serão discutidos a seguir.

Em primeiro lugar, os custos de transação no mercado imobiliário são muito altos. No caso do Brasil, uma transação de venda de imóvel custa ao vendedor cerca de $6 \%$ sobre o valor da venda a título de comissão do corretor de imóveis. Há, ainda, o imposto sobre a renda e proventos de qualquer natureza (IR) incidente sobre a valorização do imóvel vendido, cuja alíquota é de $15 \%^{1}$. O IR incide sobre a diferença entre o valor nominal pelo qual o imóvel foi adquirido e o valor nominal pelo qual foi vendido, sujeito a fatores de redução definidos no art. 39, §1 ${ }^{\circ}$ da Lei 11.196/2005 [5]. É possível demonstrar que esses fatores de redução foram definidos de forma a resultar, em geral, em bases de cálculo maiores que aquelas que seriam obtidas usando-se a diferença entre os valores reais de venda e de compra.

Por outro lado, se o indivíduo pretende vender o seu imóvel para comprar outro, poderá ser beneficiado pela hipótese de isenção de IR prevista no art. 39 da Lei 11.193/05: se os recursos obtidos com a venda do imóvel forem integralmente utilizados na compra de outro imóvel em até 180 dias após a venda, os ganhos obtidos com a venda serão isentos de IR. Eventual diferença positiva entre o valor do imóvel vendido e do imóvel adquirido será tributada. Este benefício só pode ser utilizado uma vez a cada 5 anos, pois o seu objetivo não é viabilizar arbitragem ou especulação com imóveis, mas apenas a troca de imóveis pelas famílias com

\footnotetext{
${ }^{1}$ A alíquota é definida na Instrução Normativa SRF no 84/2001 [4].
} 
finalidade de moradia. Apesar da isenção de IR, na operação de compra, o indivíduo pagará o imposto sobre a transmissão de bens imóveis (ITBI) ${ }^{2}$, os emolumentos do cartório de registro de imóveis ${ }^{3}$ e, caso o imóvel seja financiado, também as taxas cobradas pela instituição financeira (IF) que fará o financiamento.

A arbitragem entre aluguel e propriedade é dificultada pela legislação tributária, já que um indivíduo que pretende vender o seu imóvel para alugar outro terá que pagar o IR sobre a valorização do imóvel vendido, mas não terá direito a abater da base de cálculo do IR os custos que terá com o aluguel.

Além dos altos custos de transação no mercado imobiliário, e da assimetria na tributação, os bens negociados nesse mercado são, ao mesmo tempo, um investimento e um bem de consumo para as famílias [7]. Para representar essas duas características, alguns modelos consideram que a propriedade do imóvel é equivalente ao serviço de moradia que ele (potencialmente) presta ao seu proprietário. Porém, por ser um bem de consumo, as escolhas dos indivíduos entre consumir ou não esse bem, ou quanto consumir, é idiossincrática. Além disso, a escolha sobre quanto consumir depende da natureza do imóvel: indivíduos que moram em imóveis alugados têm preferências de consumo diferentes de indivíduos que têm a propriedade do imóvel onde moram. Este fato foi comprovado por Gleaser e Gyourko [2], que discutem como as hipóteses de indiferença entre localização dos imóveis e entre aluguel ou compra não são válidas para o mercado imobiliário.

Ainda, a hipótese de não-arbitragem entre aluguel e propriedade não é válida neste mercado porque os indivíduos têm preferência por possuir, e não alugar, o imóvel onde moram, já que a propriedade do imóvel oferece um hedge contra as variações no preço do aluguel e contra o risco de o proprietário do imóvel requerer a sua desocupação, forçando o locador a buscar outro imóvel. Essa aversão ao risco também é uma característica idiossincrática, como foi observado por Sinai e Souleles [8], que utilizaram um modelo de regressão para estimar o prêmio de risco de variação no preço do aluguel que estaria embutido no preço dos imóveis nos Estados Unidos, concluindo que o valor deste prêmio depende de fatores como a idade do indivíduo, o tempo que o indivíduo espera permanecer no imóvel, o comprometimento da sua renda com moradia, além de fatores não diretamente relacionados ao risco, como renda, grupo étnico, ocupação, idade e estado civil.

O mercado imobiliário é ainda caracterizado pela rigidez de preços quando há tendência de redução dos preços. Deste fato resulta que, diferentemente do que ocorre com ativos

\footnotetext{
${ }^{2}$ O ITBI é um imposto municipal incidente sobre a venda de imóveis cuja alíquota, no município de São Paulo, é atualmente de $3 \%$.

${ }^{3}$ Os emolumentos são definidos pela legislação estadual e podem ou não ser progressivos com o valor do imóvel. A título de ilustração, o registro da escritura de um imóvel de R \$700 mil custa, no estado de São Paulo, $\mathrm{R} \$ 3.358,40$ [6], o que corresponde a $0,48 \%$ do valor do imóvel.
} 
financeiros, a liquidez diminui quando os preços apresentam tendência de queda. Essa característica foi observada empiricamente por Stein [9] usando dados dos Estados Unidos, e por Ortalo-Magné e Rady [10] usando dados do Reino Unido.

Essas peculiaridades do mercado imobiliário dificultam o uso de modelos tradicionais de formação de preços. Assim, o uso de um modelo baseado em agentes heterogêneos interagentes neste trabalho terá como objetivo reproduzir a dinâmica dos preços neste mercado, que é complexa, a partir da modelagem do comportamento dos seus agentes, bem mais simples.

\subsubsection{Funcionamento do mercado fora do Brasil}

A maior parte dos trabalhos sobre a formação de preços no mercado imobiliário propõem modelos de regressão, como o proposto por Netto et al. [11], ou modelos determinísticos para formação de preços, como por exemplo o modelo proposto por Dieci et al. [12]. Alguns modelos utilizam agentes que podem assumir um entre dois tipos de estratégia, e usam o conceito de preço fundamental baseado na hipótese de não-arbitragem entre aluguel e propriedade do imóvel $[13,14]$. Contudo, como já foi discutido na Seção 1.1, a hipótese de não-arbitragem entre aluguel e propriedade não é aplicável ao Brasil por questões tributárias, e está sujeita a muitas restrições mesmo em outros países devido às preferências dos agentes pela propriedade em detrimento do aluguel, como foi discutido na seção 1.1.

Sommervoll et al. [15] propõem um modelo baseado em agentes heterogêneos para a formação de preços no mercado imobiliário. Nesse modelo, a hipótese de não-arbitragem entre aluguel e propriedade do imóvel não é usada, e todos os agentes usam a mesma estratégia, porém com componentes aleatórios que os distinguem entre si. O mercado é construído como um leilão, em que os compradores dão lances (bids) e os vendedores decidem por realizar a venda quando o maior lance recebido for maior que o seu preço de reserva. A cada instante, os valores das transações realizadas são usados para compor o índice de preços daquele instante, $I_{t}$.

Os compradores são agentes heterogêneos que definem o seu preço de reserva maximizando uma função utilidade que depende do consumo de moradia e de outros bens, e da expectativa de valorização futura do imóvel, $U=c_{t}+h_{t}+\alpha_{i}(r) \sqrt{h_{t}}$, em que $h_{t}$ é a quantidade consumida de moradia, $c_{t}$ é a quantidade consumida de outros bens, $r$ é a taxa esperada de valorização dos imóveis e $\alpha_{i}(r)>0$ é uma função tal que $\alpha_{i}(0)=0$. A primeira parte da função utilidade, $h_{t}$, representa o aspecto "bem de consumo" da moradia, enquanto que a segunda parte, $\alpha_{i}(r) \sqrt{h_{t}}$, representa o aspecto "investimento".

Os compradores estão sujeitos também a uma restrição orçamentária $Y=p_{c t} c_{t}+p_{h t} h_{t}$, em que $Y$ é a renda dos agentes, $p_{h t}$ é o preço de uma unidade de moradia e $p_{c t}$ é o preço de uma 
unidade de outros bens. Com essa utilidade, os compradores definem o seu preço de reserva, $p_{h t}^{*}$, para consumir uma unidade de moradia.

Os compradores também fazem uma previsão do valor do imóvel no próximo instante, segundo a função $u_{i}\left(I_{t-1}+\Delta I_{t-1}\right)$, em quem $\Delta I_{t}=I_{t}-I_{t-1}$ e $u_{i}$ é uma variável aleatória (VA) com distribuição uniforme e $E\left(u_{i}\right)=1$. O valor do bid dado por cada comprador é o mínimo entre o preço de reserva, $p_{h t}^{*}$, e o valor previsto para o índice no próximo instante:

$$
\operatorname{Bid}_{i}=\min \left\{p_{h t}^{*}, u_{i}\left(I_{t-1}+\Delta I_{t-1}\right)\right\}
$$

Portanto, os compradores são heterogêneos quanto ao peso dado à expectativa de valorização do imóvel, $\alpha_{i}(r)$, e também quanto ao multiplicador da variação, $u_{i}$, mas não são heterogêneos quanto às preferências de consumo. Além disso, a renda dos compradores, $Y$, é a mesma para todos os agentes.

A restrição de crédito aos compradores, quando é incluída no modelo, considera o limite de renda dos compradores, mas não as restrições sobre um percentual mínimo de entrada no financiamento. Em relação aos valores de entrada, os autores atribuem uma distribuição ao valor de riqueza inicial de que cada comprador dispõe:

$$
F(x)=\left\{\begin{array}{ll}
0, & x<0 \\
\frac{1}{2}, & x=0 \\
\frac{1}{2}(x+1), & 0<x \leq 1 \\
1, & x>1
\end{array} .\right.
$$

Como será discutido na Seção 1.2.2, o percentual mínimo de entrada no financiamento tem efeito muito significativo no risco da operação do ponto de vista da IF que faz o financiamento, além de ser também uma restrição para obtenção do financiamento no caso do Brasil. Por isso, no modelo proposto neste trabalho, a riqueza e a renda inicial dos compradores serão tratadas de outra forma.

Os vendedores são agentes com preço de reserva homogêneo $a I_{t}$, com $0<a \leq 1$ constante. A cada instante, caso um vendedor não consiga realizar a venda, i.e., não receba um bid maior que o seu preço de reserva, ele reajusta o seu preço de reserva para um valor menor, $a^{n} I_{t}$, com $n$ o número de vezes em que não houve bid maior que o preço de reserva. A regra de reajuste também é a mesma para todos os vendedores.

A dinâmica dos preços é obtida por meio de simulações. Os autores obtiveram dinâmicas de preços cíclicas, com comprimento de onda e amplitude dependentes dos valores dos parâmetros usados. Porém, o trabalho não mostra se é possível ocorrer outras situações, como pontos de equilíbrio estáveis, ou dinâmicas instáveis, a depender dos valores dos parâmetros. 


\subsubsection{Funcionamento do mercado no Brasil}

O modelo de Sommervoll et al., descrito na Seção 1.1.1, utiliza algumas hipóteses que não se aplicam ao mercado brasileiro. Em primeiro lugar, a hipótese de renda constante não é válida, pois cada família tem uma renda diferente, o que limita o valor que pode ser financiado por ela. Além disso, a restrição quanto ao valor de entrada do financiamento imobiliário também é fundamental, tanto por ser um critério para a obtenção do financiamento pelas famílias, como também por ser um fator determinante para a definição do risco da operação.

A estrutura do mercado em leilão também não reflete corretamente a realidade brasileira. No Brasil, o mercado imobiliário funciona como um leilão reverso, i.e., cada vendedor anuncia o seu imóvel por um valor fixo (ask), e os compradores encaminham propostas de valores próximos, porém menores, do que o valor anunciado pelo vendedor. Caso os vendedores percebam que a demanda por imóveis está diminuindo, podem ajustar o preço dos seus imóveis para baixo, a depender da pressa que têm para realizar a venda. Na situação contrária, caso percebam um aumento da procura por imóveis, podem pedir valores maiores.

Por essas características, a dinâmica do mercado brasileiro se assemelha, do ponto de vista dos vendedores, ao funcionamento da bolsa de valores. Num sistema de bolsa, os vendedores registram as ordens no livro de ofertas e realizam a venda caso existam ordens de compradores por valores maiores, sendo que as transações, quando ocorrem, são fechadas pelo valor de ask. Silva [16] propõe um modelo de agentes para o livro de ofertas em que o valor de ask dos agentes fundamentalistas é dado por uma soma de duas variáveis aleatórias com distribuição normal, mas com parâmetros diferentes:

$$
P_{i}=f_{i}+\mu_{i}
$$

com $f_{i} \sim \mathcal{N}\left(f_{M}, \sigma_{f}\right)$ representando o preço fundamental do papel e $\mu_{i} \sim \mathcal{N}\left(\mu_{M}, \sigma_{\mu}\right)$ representando a paciência do agente em esperar para realizar a venda. $O$ conceito de paciência do agente em esperar para realizar a venda representando as idiossincrasias dos vendedores será aplicado no modelo deste trabalho.

No presente trabalho, propomos um modelo para a formação de preços no mercado imobiliário que traz alguns elementos do modelo proposto por Sommervoll et al. [15], porém adaptado à realidade do mercado brasileiro, que funciona de maneira similar ao proposto por Silva [16]: os vendedores são heterogêneos na pressa que têm em realizar a venda. Os aspectos relacionados ao crédito e à heterogeneidade da renda e do patrimônio dos compradores também são tratados de forma a representar as características do mercado brasileiro. 


\subsection{O financiamento imobiliário no Brasil}

O funcionamento do mercado imobiliário depende significativamente das regras de financiamento a ele aplicáveis. Isso acontece porque o valor dos imóveis é normalmente muito maior do que a riqueza das famílias e, por isso, elas dependem da disponibilidade de financiamento para entrar nesse mercado [10].

Os imóveis destinados a moradia e as operações de financiamento imobiliário representam um valor muito alto em relação ao patrimônio e a renda das famílias. Por isso, tipicamente esses financiamentos têm prazo muito longo. No Brasil, as taxas de juros reais ${ }^{4}$ praticadas no mercado de crédito de taxas livres ${ }^{5}$ a pessoas físicas são muito altas, encontrando-se em torno de 40\%a.a. [17]. Operações de crédito a essas taxas e com prazos muito longos se tornam inviáveis, no sentido de que as famílias não teriam renda suficiente para suportar as parcelas resultantes de tais operações. Por isso, o financiamento imobiliário recebe um tratamento regulatório específico, com o objetivo de viabilizar operações e incentivar o crescimento do setor. Os principais meios usados para viabilizar essas operações são os direcionamentos obrigatórios de recursos captados pelas IFs em depósitos de poupança e dos recursos do Fundo de Garantia por Tempo de Serviço (FGTS), que serão brevemente descritos a seguir.

\subsubsection{Direcionamento obrigatório da poupança}

O custo de captação das IFs depende de vários fatores, dentre os quais a taxa Selic ${ }^{6}$. A taxa que um banco cobrará do cliente numa operação de crédito será o seu custo de captação somado ao spread bancário, o que, no Brasil, resulta em taxas muito altas. Para reduzir a taxa das operações de financiamento imobiliário, o governo, por meio do Conselho Monetário Nacional ( $\mathrm{CMN})$, determina o direcionamento obrigatório dos depósitos da poupança ao crédito imobiliário.

Em 1861, um decreto do Imperador Dom Pedro II instituiu a Caixa Econômica Federal e Monte de Socorro, autorizada a operar a caderneta de poupança e o penhor. Este decreto também definia que a taxa de remuneração dos depósitos da poupança seria de $6 \%$ [19].

\footnotetext{
${ }^{4} \mathrm{~A}$ taxa de juros real é a taxa de juros nominal descontada a inflação.

${ }^{5} \mathrm{O}$ mercado de taxas livres não inclui as operações com recursos direcionados como, por exemplo, operações de crédito rural e de crédito imobiliário no âmbito do SFH, sendo que estas últimas serão discutidas nesta seção.

${ }^{6} \mathrm{~A}$ taxa Selic é taxa de juros básica no Brasil, cuja meta é definida pelo Comitê de Política Monetária e, em setembro de 2015, estava fixada em $14,25 \%$ ao ano. Ela é formada pela média das taxas das operações compromissadas de um dia cursadas no Selic, com lastro em títulos públicos federais, conforme definido na Circular вСв 3.671/2013 [18].
} 
Hoje, a poupança é uma modalidade de captação de depósitos a prazo operada pelas instituições que fazem parte do Sistema Brasileiro de Poupança e Empréstimo ( $\mathrm{SBPE}^{7}$ ), cujo principal agente é a Caixa Econômica Federal (CEF). A taxa de remuneração dos depósitos de poupança continua sendo muito baixa: $8,57 \%$ a.a., contra $14,25 \%$ a.a. da taxa Selic (meta) em 18 de setembro de $2015^{8}$. Portanto, ela é uma fonte de captação muito barata para as IFs.

Em contrapartida ao baixo custo de captação, as IFs integrantes do SBPE devem obrigatoriamente direcionar parte dos recursos dessa captação a operações de financiamento imobiliário com taxa de juros máxima de $12 \%$ a.a. ${ }^{9}$, definida pela Resolução CMN 3.932/10 [21].

Esse direcionamento é dito obrigatório porque, caso não seja feito, ou seja, se a IF não realizar o volume de operações de financiamento imobiliário correspondente às suas captações de poupança, a diferença não pode ser usada para realizar operações a taxas de mercado (superiores a $12 \%$ a.a.), e deve ser recolhida ao Banco Central do Brasil (BCB), com remuneração de apenas $80 \%$ da taxa de captação da poupança.

\subsubsection{O Sistema Financeiro de Habitação}

O Sistema Financeiro de Habitação $(\mathrm{SFH})$ está definido no art. $1^{\circ}$ do regulamento anexo à Resolução CMN 1.980/93: "[i]ntegram o Sistema Financeiro da Habitação (SFH), na qualidade de agentes financeiros, os bancos múltiplos com carteira de crédito imobiliário, as caixas econômicas, as sociedades de crédito imobiliário, as associações de poupança e empréstimo, as companhias de habitação, as fundações habitacionais, os institutos de previdência, as companhias hipotecárias, as carteiras hipotecárias dos clubes militares, as caixas militares, os montepios estaduais e municipais e as entidades de previdência complementar".

A Resolução CMN 3.932/10 diferencia os financiamentos imobiliários entre aqueles feitos no âmbito do SFH e aqueles feitos a taxas de mercado. A diferença entre eles são as restrições aplicáveis aos financiamentos no âmbito do SFH, que não se aplicam aos financiamentos imobiliários a taxas de mercado, a saber ${ }^{10}$ :

- taxa máxima de juros de $12 \%$ ao ano;

\footnotetext{
${ }^{7} \mathrm{O}$ art. $2^{\circ}$ do regulamento anexo à Resolução CMN 1.980/93 [20] define que "[o] sistema brasileiro de poupança e empréstimo (SBPE) é integrado pelos bancos múltiplos com carteira de crédito imobiliário, pelas caixas econômicas, pelas sociedades de crédito imobiliário e pelas associações de poupança e empréstimo".

${ }^{8}$ Maiores informações sobre as regras de remuneração da poupança se encontram na seção A.1.

${ }^{9} \mathrm{~A}$ regra completa sobre o direcionamento dos recursos da poupança é apresentada na seção A.2.

${ }^{10}$ regras válidas em agosto de 2015
} 
- razão entre o valor financiado e o valor do imóvel, i.e., loan to value (LTV) máximo de $80 \%$, ou de $90 \%$ se o financiamento for contratado usando o Sistema de Amortizações Constantes (SAC) e

- valor máximo do imóvel financiado de R\$650 mil ${ }^{11}$.

Além dessas restrições, a Lei 8.692/93 estabelece que os saldos devedores dos financiamentos no âmbito do SFH serão reajustados à TR e que o comprometimento máximo de renda mensal com o pagamento do financiamento deve ser de $30 \%$ [22] .

Em relação às operações no âmbito do SFH, as IFs têm liberdade para cobrar taxas de juros menores que a máxima definida na resolução, assim como adotar limites de LTV e de comprometimento de renda mais restritivos que os máximos definidos na legislação.

Note que as condições para que um financiamento possa ser feito no âmbito do SFH têm, por um lado, o objetivo de facilitar a obtenção de financiamento para as famílias, garantindo taxas de juros mais baixas, principalmente para aquelas que estão comprando o primeiro imóvel (impondo um limite máximo ao valor do imóvel a ser financiado). Por outro lado, a imposição de limites máximos de LTV e comprometimento de renda tem como objetivo garantir a qualidade creditícia das operações originadas nessas condições.

Diversos trabalhos apresentam evidências empíricas de que valores maiores de LTV estão associados a maior risco de inadimplência das operações e a perdas maiores nas operações de financiamento imobiliário. Campbell e Dietrich [23] analisaram dados de 2,5 milhões de contratos de financiamento imobiliário, nos Estados Unidos, segurados pela Mortgage Guaranty Insurance Corporation, e observaram que a incidência de default está significativamente relacionada tanto ao LTV original da operação como também ao current loan to value (CLTV), indicando que financiamentos com potencial para amortização negativa também têm risco maior de default. Este fato está empiricamente consistente com a distinção feita nas regras do SFH entre operações feitas pelo sistema Price (LTV máximo de 80\%), que têm potencial de amortização negativa, e aquelas feitas pelo SAC (LTV máximo de 90\%), em que isso não acontece.

Quigley e Order [24], usando dados de 300.000 contratos de financiamentos imobiliários originados entre 1976 e 1980 nos Estados Unidos, comprados pela Federal Home Loan Mortgage Corporation (Freddie Mac), estimaram a curva de sobrevivência dos contratos para (a) LTV < $81 \%$, (b) $81 \% \leq$ LTV $\leq 90 \%$ e (c) LTV > 90\% e mostram que as operações com LTV maior são significativamente mais arriscadas.

Wong et al. [25], usando dados do mercado de crédito imobiliário de Hong Kong, observaram que valores maiores de CTLV estão associados a maior probabilidade de default nas

\footnotetext{
${ }^{11}$ exceto no DF e nos estados de SP, RJ, MG, onde o valor máximo é de R\$750 mil.
} 
operações de financiamento imobiliário. Qi e Yang [26], usando dados históricos de seguradoras de crédito imobiliário nos Estados Unidos, concluíram que o principal fator associado a perdas maiores dada a ocorrência de inadimplência é o CLTV da operação.

\subsubsection{Fundo de Garantia do Tempo de Serviço (FGTS)}

O FGTS é um fundo, hoje regido pela Lei 8.036/90 [27], que constitui uma espécie de "poupança forçada" dos trabalhadores. A CEF é o agente operador do fundo. Têm direito ao FGTS os trabalhadores com contratos regidos pela Consolidação das Leis do Trabalho (CLT), trabalhadores avulsos e diretores não empregados. A principal fonte de recursos do FGTS são os depósitos feitos pelos empregadores nas contas vinculadas dos empregados, à alíquota de $8 \%$ sobre os rendimentos do trabalho. Esses recursos são remunerados à taxa extremamente baixa de $3 \%$ a.a., e corrigidos pela TR.

Em geral, os titulares dessas contas vinculadas (os trabalhadores) não podem movimentar as contas, o que justifica a definição de "poupança forçada". Os recursos guardados nessas contas podem ser movimentados pelos titulares apenas nas situações listadas no art. 20 da Lei 8.036/90, dentre as quais listamos apenas aquelas que são relevantes para o financiamento imobiliário a pessoas físicas:

- pagamento de prestações de financiamentos feitos no âmbito do SFH (inciso V);

- amortização ou liquidação de financiamentos feitos no âmbito do SFH (inciso VI) e

- compra de imóvel que poderia ser financiado no âmbito do SFH, ainda que o financiamento não ocorra (inciso VII).

Por ser uma poupança forçada, e pela existência dessas hipóteses em que esses recursos podem ser usados para comprar um imóvel, os depósitos que os trabalhadores têm na sua conta vinculada do FGTS se tornam uma importante fonte de recursos para dar entrada (downpayment) na compra do imóvel.

Note que, nas três situações listadas, o uso dos recursos do FGTS para a compra de um imóvel está restrito aos casos em que as características do imóvel e do seu financiamento (se houver) os colocam no âmbito do SFH. Assim, as regras de financiamento de imóveis no âmbito SFH são relevantes não só pelas suas taxas de juros reduzidas (ver subseção 1.2.1), mas também pela disponibilidade de recursos para dar como entrada no financiamento imobiliário, já que os imóveis que não se enquadram nesses limites não podem ter o downpayment oriundo dos recursos poupados em conta vinculada do FGTS. 


\subsubsection{Carteira hipotecária e outros limites}

Nas Seções 1.2.1 e 1.2.3, vimos que, do ponto de vista do comprador, é interessante que o financiamento seja feito no âmbito do SFH, por vários motivos, a saber:

- taxas de juros menores que a dos financiamentos a taxas de mercado e

- possibilidade de utilizar os recursos poupados em conta vinculada do FGTS como entrada do financiamento.

No entanto, as IFs podem, ainda, realizar financiamentos imobiliários a taxas de mercado (não sujeitas, portanto, às regras do SFH). Tais operações seguem as disposições da Lei 9.514/1997 [28], que trata do Sistema de Financiamento Imobiliário (SFI), e compõem a chamada carteira hipotecária. Os recursos da conta vinculada do FGTS não podem ser utilizados pelos mutuários nesse tipo de financiamento imobiliário. Essas operações recebem uma fração muito pequena do direcionamento da poupança (no máximo $13 \%$ - ver seção A.2), mas podem também ser feitas com outros tipos de captação, sem direcionamento obrigatório. Nessas operações, os limites impostos para LTV, comprometimento de renda, e valor máximo do imóvel financiado, se houver, serão definidos na política de concessão de crédito definida pela IF, e variam entre as instituições.

As IFs impõem ainda outros limites, como o limite de idade do comprador e o limite de prazo máximo $^{12}$ do financiamento, tanto para operações no âmbito do SFH como para operações a taxas de mercado. O limite de prazo máximo do financiamento tem impacto direto no valor das prestações, influenciando fortemente a disponibilidade de crédito para a população.

Por terem prazo longo, as operações de financiamento imobiliário criam uma perspectiva de relacionamento muito longo entre o cliente e a IF que fez a operação. Por isso, apesar das taxas pouco atraente, mesmo IFs privadas algumas vezes optam por trabalhar nesse mercado. Ainda assim, observamos na Tabela 1.1 que, no Brasil, 75, 7\% do total de crédito imobiliário no Brasil é detido pela CEF, e esse percentual aumenta para $82,3 \%$ quando considerados a CEF e o Banco do Brasil, também controlado pela União. A CEF é, portanto, o maior agente operador do SFH do Sistema Financeiro Nacional (SFN), e os limites por ela praticados acabam sendo o paradigma para as demais IFs que pretendem realizar operações nesse mercado.

\footnotetext{
${ }^{12}$ Até 1997, o art. $4^{\circ}$, III do Regulamento Anexo à Resolução CMN 1.980/93 limitava o prazo máximo dos financiamentos no âmbito do SFH em 20 anos. Essa restrição foi revogada pela Resolução CMN 2.458/97.
} 
Tabela 1.1: Percentual do volume de financiamento imobiliário no SFN na data-base março/2015.

\begin{tabular}{cc}
\hline IF ou conglomerado & $\begin{array}{c}\text { percentual do volume total de } \\
\text { financiamento imobiliário }\end{array}$ \\
\hline \hline CEF & $75,66 \%$ \\
Banco do Brasil & $6,66 \%$ \\
Itaú & $6,01 \%$ \\
Santander & $5,04 \%$ \\
Bradesco & $4,03 \%$ \\
HSBC & $1,22 \%$ \\
Banrisul & $0,59 \%$ \\
APE Poupex & $0,21 \%$ \\
Citibank & $0,16 \%$ \\
BRB & $0,11 \%$ \\
\hline
\end{tabular}

Fonte: Elaboração própria a partir de relatório do DW-SCR de dados do documento 3040, disponível em https://www3.bcb.gov.br/informes/relatorios?lingua=pt.

\subsection{Justificativa}

O modelo proposto neste trabalho tem o objetivo de descrever a dinâmica de preços no mercado imobiliário no Brasil. Os preços dos imóveis estão diretamente relacionados ao crédito imobiliário, em dois sentidos: a disponibilidade de crédito imobiliário é determinante na formação de preços no mercado imobiliário porque os imóveis representam uma fração muito significativa do patrimônio das famílias, e elas dependem do crédito para entrar nesse mercado; e, no sentido contrário, a qualidade creditícia dos financiamentos imobiliários é afetada pelo preço dos imóveis, já que são os próprios imóveis dados em garantia de tais operações.

Como já foi discutido na seção 1.2, o direcionamento obrigatório dos recursos da poupança representa a principal fonte de recursos utilizada pelas IFs no financiamento imobiliário. Considerando que no mínimo $80 \%$ dos recursos direcionados devem ser utilizados em operações no âmbito do $\mathrm{SFH}$, e ainda, que mais de $75 \%$ do financiamento imobiliário (em valor de ativos) se encontra na CEF, as regras do SFH e aquelas definidas na política interna de concessão de crédito da CEF são determinantes para a disponibilidade de crédito no país. $\mathrm{O}$ modelo tratará, portanto, das condições de financiamento do SFH, devido à sua relevância para o crédito imobiliário brasileiro.

O comportamento dos compradores no modelo será bastante simples: os compradores tentam comprar um imóvel sempre que tiverem recursos próprios ou crédito suficiente, sujeitos a sua restrição de renda e de riqueza aplicada aos limites de financiamento no SFH. Sua expectativa é modelada por seu preço de reserva, que vai se adaptando ao nível de preços observado. Os vendedores definem o valor pelo qual anunciarão seu imóvel de acordo com a sua pressa, com 
o nível de preços observado e também com a sua percepção sobre a situação do mercado. Veremos que, com esta construção, o preço de equilíbrio do mercado emerge como função dos seguintes fatores:

- as características dos compradores, a saber, sua distribuição de renda e riqueza e preço de reserva;

- as características dos vendedores, i.e., sua pressa, memória e sensibilidade à situação do mercado;

- a proporção de compradores e vendedores que, no modelo, representa a disponibilidade relativa de imóveis;

- as condições para obtenção de financiamento no âmbito do SFH.

Assim, o modelo mostra como mudanças nas condições de financiamento alteram o preço de equilíbrio. Porém, diferentemente dos modelos lineares normalmente usados neste tipo de modelagem, este modelo terá uma dinâmica não linear de segunda ordem. Assim, com ele é possível não só obter o preço de equilíbrio, mas também a dinâmica da convergência do preço, i.e., o caminho que o preço irá percorrer até o valor de equilíbrio, quando ocorre uma mudança nas condições do mercado. Essa dinâmica de segunda ordem permite que alterações nas condições do mercado façam com que o preço fique acima (ou abaixo) do preço de equilíbrio durante a convergência, o que caracteriza a formação de bolhas. Além disso, a não-linearidade do modelo permite ainda o surgimento de uma dinâmica oscilatória estável para o preço, quando o preço oscila mesmo sem haver modificação nas condições do mercado. 


\section{Capítulo 2}

\section{Definição do modelo}

O modelo de formação de preços no mercado imobiliário proposto neste trabalho será construído partindo do comportamento dos agentes compradores e dos agentes vendedores para obter a dinâmica do preço de mercado dos imóveis. O objetivo do modelo é mostrar como mudanças nas variáveis do SFH afetam a dinâmica do preço de mercado e o preço de equilíbrio.

\subsection{Definição do mercado}

O modelo proposto neste trabalho é a tempo discreto. O preço de mercado dos imóveis, no instante $t \in \mathbb{N}$, será definido como a média do valor das transações ocorridas no instante $t$ :

$$
\boldsymbol{P}_{t}=\frac{1}{\boldsymbol{n}_{t}} \sum_{k=1}^{\boldsymbol{n}_{t}} \boldsymbol{T}_{t}^{(k)}
$$

em que $\boldsymbol{P}_{t}$ é o preço de mercado no instante $t, \boldsymbol{n}_{t}$ é o número de transações ocorridas no instante $t$ e $\boldsymbol{T}_{t}^{(k)}$ é o valor pelo qual foi fechada a $k$-ésima transação no instante $t$.

O estoque $S$ de imóveis é constante no tempo. O mercado é formado por $C+V$ agentes, sendo $C$ compradores e $V$ vendedores, e esses números são constantes ao longo do tempo. Ao realizar uma compra, o agente comprador se torna vendedor, enquanto que o vendedor se torna comprador.

Nem todos os agentes que possuem imóvel têm intenção de vendê-lo, porque muitos pretendem apenas consumir o serviço de moradia que o seu imóvel proporciona, e não estão negociando seu imóvel. Para tratar esses proprietários que não têm intenção de vender o imóvel, introduzimos a Hipótese 1. 
Hipótese 1. Vamos considerar que todo proprietário é um potencial vendedor, no sentido de que aqueles proprietários que não querem vender seu imóvel estarão dispostos a vendê-lo apenas por valores muito acima do preço de mercado. Assim, vamos considerar $S=V$.

Num dado instante de tempo $t$, cada vendedor $i$ anuncia seu imóvel no mercado por um preço idiossincrático, $\boldsymbol{A}_{t}^{(i)}$, que será definido na seção 2.3. Esse valor do anúncio depende da pressa do vendedor, do preço de mercado no instante anterior e também da demanda histórica.

Já os compradores têm um preço de reserva ${ }^{1}$, que é o valor máximo que aceitam pagar pelo imóvel. Para entrar no mercado no instante $t$, o comprador precisa conseguir os recursos correspondentes ao seu preço de reserva. Esses recursos podem ser oriundos da riqueza do próprio comprador ou de financiamento. Caso consiga os recursos, o comprador entrará no mercado e tentará comprar um imóvel de preço menor ou igual ao seu preço de reserva. Se não conseguir, ele fica fora do mercado. Note que o comprador não pode entrar no mercado com um valor menor que o preço de reserva para fechar uma transação, mesmo que tenha um valor menor disponível para realizar a compra. Isso nos leva a formular a seguinte hipótese:

Hipótese 2. Um comprador que, no instante t, conseguir menos recursos do que o seu preço de reserva, ficará fora do mercado naquele instante.

Obviamente, a Hipótese 2 simplifica o comportamento dos compradores pois, na realidade, se houver imóveis anunciados por menos que o preço de reserva dos compradores, um comprador que consegue recursos em valor inferior ao preço de reserva pode encontrar um imóvel dentro da sua disponibilidade de recursos, e não fica obrigatoriamente fora do mercado. A formulação do comportamento dos compradores será detalhado na seção 2.2.

Uma transação ocorre quando um comprador que entrou no mercado encontra um vendedor que anunciou por um valor menor ou igual ao preço de reserva do comprador. Ela será sempre fechada pelo valor anunciado pelo vendedor, e não pelo preço de reserva do comprador:

Hipótese 3. Quando ocorre uma transação, ela é sempre fechada pelo valor anunciado pelo vendedor que participou daquela transação, e não pelo preço de reserva do comprador.

No momento em que as transações são fechadas, pode ocorrer uma de duas situações:

\footnotetext{
${ }^{1}$ A definição de preço de reserva pelo lado da demanda é consistente com a premissa de que o mercado funciona como um leilão reverso. Por isso, são os compradores, e não os vendedores, que estabelecem o limite de preço (neste caso, máximo) pelo qual aceitam fechar transação. Por simplicidade, chamaremos este limite de preço apenas de "preço de reserva" ou "preço de reserva dos compradores". É importante não confundi-lo com a definição usual de preço de reserva, aplicável ao sistema de leilão comum e que denota o valor mínimo pelo qual o vendedor aceita vender o bem, e que não será utilizada neste trabalho.
} 
1. Há mais compradores que entraram no mercado do que vendedores que anunciaram por valores menores que o preço de reserva: nesse caso, todos os vendedores que anunciaram por menos que o preço de reserva dos compradores fecharão transação, e alguns compradores que entraram no mercado não conseguirão comprar imóvel.

2. Há mais vendedores que anunciaram por valores menores que o preço de reserva do que compradores que entraram no mercado: nesse caso, todos os compradores que conseguiram entrar no mercado conseguirão comprar um imóvel, mas alguns vendedores que anunciaram por valores menores que o preço de reserva dos compradores não conseguirão fazer a venda. Esses compradores escolherão o vendedor, dentre aqueles que anunciaram por menos que o seu preço de reserva, de forma aleatória, i.e., todos os vendedores que anunciaram por valor menor que o preço de reserva dos compradores têm igual probabilidade de fecharem uma transação, independentemente do valor do anúncio.

No instante $t$, todos os agentes têm informação sobre o valor do preço de mercado e sobre a demanda no passado, de forma agregada. São essas as informações que os vendedores utilização para definir o valor do anúncio e que os compradores utilização para definir o seu preço de reserva no instante $t$. Esta característica será fundamental para obter as propriedades do modelo, e pode ser resumida na seguinte hipótese:

Hipótese 4. No instante t, os agentes não têm acesso às informações sobre o comportamento dos demais no instante t. As únicas informações disponíveis aos agentes no instante $t$ são o preço de mercado e a demanda passados.

Nas seções seguintes, vamos mostrar, de forma detalhada, o comportamento dos agentes compradores (Seção 2.2), dos agentes vendedores (Seção 2.3), e como o preço é formado a partir das transações (Seção 2.5).

\subsection{Compradores}

Há $C$ compradores no mercado. Cada comprador $j, j=1, \ldots, C$, tem uma renda $\boldsymbol{Y}^{(j)}$ e uma riqueza $\boldsymbol{W}^{(j)}$. Vamos considerar que a renda $\boldsymbol{Y}^{(j)}$ e a riqueza $\boldsymbol{W}^{(j)}$ são variáveis aleatórias não negativas, com densidade conjunta $f_{Y, W}(y, w)$.

Hipótese 5. Os vetores aleatórios $\left(\boldsymbol{Y}^{(j)}, \boldsymbol{W}^{(j)}\right), j=1, \ldots, C$, são independentes.

Em cada instante $t \in \mathbb{N}$, os compradores têm todos o mesmo preço de reserva igual a $b \boldsymbol{P}_{t-1}$, com $b>0$ constante. Os compradores podem usar o valor do preço de mercado de instantes 
passados para apurar o seu preço de reserva porque, pela Hipótese 4, o preço de mercado apurado no passado está disponível a todos os agentes. O parâmetro $b$ representa o quanto a mais (ou a menos, se $b<1$ ) os compradores estão dispostos a pagar no instante $t$ em relação ao preço de mercado que foi apurado no instante anterior.

Lembramos que cada comprador tenta obter o valor do seu preço de reserva, $b \boldsymbol{P}_{t-1}$, para comprar o imóvel. Caso consiga, ele terá até $b \boldsymbol{P}_{t-1}$ para comprar um imóvel; se não conseguir, pela Hipótese 2, ficará fora do mercado.

Assim, o bid de cada comprador, i.e., o valor que o comprador $j$ tem disponível no intervalo $t$ para comprar um imóvel é

$$
\boldsymbol{B}_{t}^{(j)}= \begin{cases}b \boldsymbol{P}_{t-1}, & \text { se } j \text { obtém os recursos } \\ 0, & \text { c.c. }\end{cases}
$$

Seja $\boldsymbol{J}_{t}^{(j)}$ uma variável aleatória indicadora se o comprador $j$ obtém ou não os recursos para entrar no mercado:

$$
\boldsymbol{J}_{t}^{(j)}= \begin{cases}1, & \text { se } j \text { obtém os recursos } \\ 0, & \text { c.c. }\end{cases}
$$

De (2.2.1) e (2.2.2), temos que

$$
\boldsymbol{B}_{t}^{(j)}=b \boldsymbol{P}_{t-1} \boldsymbol{J}_{t}^{(j)}
$$

Para obter a função de distribuição de $\boldsymbol{B}_{t}^{(j)} \mid \boldsymbol{P}_{t-1}$, precisamos conhecer as condições sob as quais o comprador consegue os recursos para entrar no mercado. Essas condições dependem da renda e da riqueza do comprador, e são descritas a seguir.

\subsubsection{Condições para obter os recursos para compra}

O comprador $j$ pode usar recursos próprios para fazer a compra ou obter parte dos recursos por meio de um financiamento. Ele usará toda a sua riqueza $\boldsymbol{W}^{(j)}$ para fazer a compra à vista ou dar entrada no financiamento, e usará a sua renda $\boldsymbol{Y}^{(j)}$ para pagar as prestações do financiamento. Contudo, caso ele necessite de financiamento, i.e., se a sua riqueza $\boldsymbol{W}^{(j)}$ for menor que o seu preço de reserva, terá que satisfazer os requisitos do SFH (ver Seção 1.2.2) para obter o financiamento. Essas condições devem ser atendidas simultaneamente e dependem da renda e da riqueza do comprador $j$. São elas: 
1. limite máximo do valor do imóvel financiado $\left(P_{\max }\right)$

$$
b \boldsymbol{P}_{t-1} \leq P_{\max }
$$

2. limite máximo de LTV $(R)$

$$
\frac{b \boldsymbol{P}_{t-1}-\boldsymbol{W}^{(j)}}{b \boldsymbol{P}_{t-1}} \leq R
$$

3. limite máximo da fração de comprometimento da renda do comprador com o pagamento das prestações mensais do financiamento $(\rho)$

$$
\begin{aligned}
\left(b \boldsymbol{P}_{t-1}-\boldsymbol{W}^{(j)}\right)\left(r+\frac{1}{\tau}\right) \leq \rho \boldsymbol{Y}^{(j)} & \\
& b \boldsymbol{P}_{t-1}-\boldsymbol{W}^{(j)} \leq \frac{1}{k} \boldsymbol{Y}^{(j)}, k=\frac{r+1 / \tau}{\rho},
\end{aligned}
$$

em que $r$ é a taxa de juros mensal do financiamento e $\tau$ é o prazo máximo de financiamento em meses.

Hipótese 6. Vamos relaxar a condição (2.2.4) e considerar no modelo apenas as condições (2.2.5) e (2.2.6) para obtenção de financiamento.

Sob a Hipótese 6, se as condições (2.2.5) e (2.2.6) forem atendidas pelo comprador $j$, ou se a sua riqueza for maior ou igual ao seu preço de reserva, temos $\boldsymbol{J}_{t}^{(j)}=1$, ou seja:

$$
\boldsymbol{J}_{t}^{(j)}=1 \Longleftrightarrow \begin{cases}(1-R) b \boldsymbol{P}_{t-1}<\boldsymbol{W}^{(j)} & \text { e } b \boldsymbol{P}_{t-1}-\boldsymbol{W}^{(j)}<\frac{1}{k} \boldsymbol{Y}^{(j)} \\ \boldsymbol{W}^{(j)}>b \boldsymbol{P}_{t-1} & \text { ou }\end{cases}
$$

Note que, quando $\boldsymbol{Y}^{(j)}>0$, a condição $\boldsymbol{W}^{(j)}>b \boldsymbol{P}_{t-1}$ (compra à vista) já está incluída na condição (2.2.6), sobre o comprometimento de renda. Como as variáveis $\boldsymbol{Y}^{(j)}$ foram definidas como não negativas, temos que

$$
\mathbb{P}\left(\boldsymbol{J}_{t}^{(j)}=1 \mid \boldsymbol{P}_{t-1}=p\right)=\mathbb{P}\left((1-R) b p<\boldsymbol{W}^{(j)} \text { e } b p-\boldsymbol{W}^{(j)}<\frac{1}{k} \boldsymbol{Y}^{(j)}\right)
$$

A Figura 2.2.1 mostra a região correspondente às restrições (2.2.5) e (2.2.6). Note que a Região I corresponde aos casos em que os compradores obtêm financiamento para entrar no 


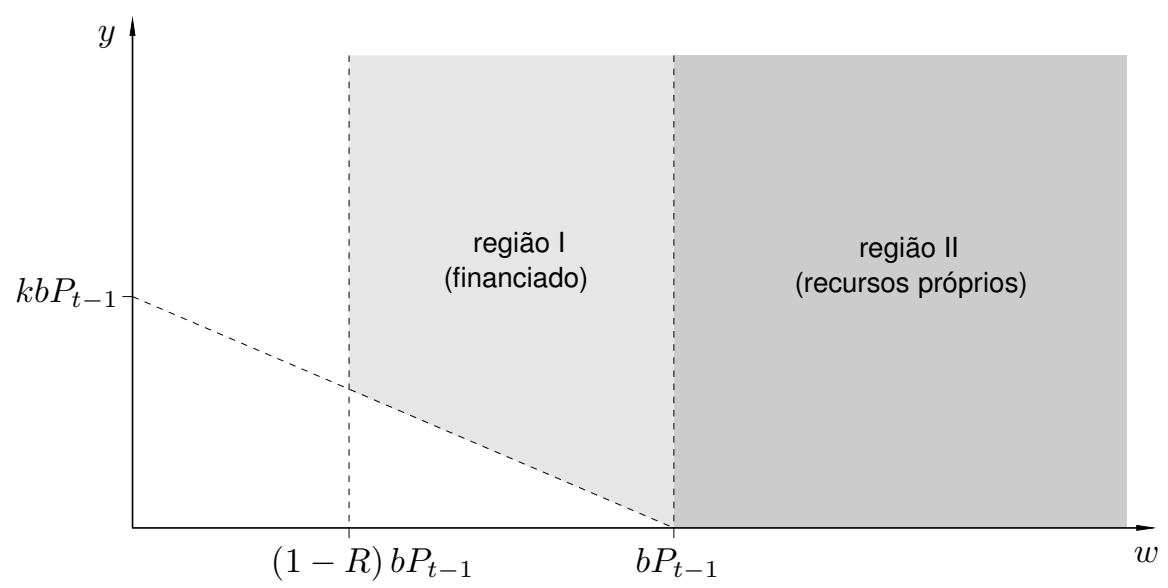

Figura 2.2.1: Região de integração para obter $\mathbb{P}\left(\boldsymbol{J}_{t}^{(j)}=1\right)$.

mercado e a Região II corresponde aos casos em que os compradores dispõem dos recursos para entrar no mercado sem a necessidade de financiamento. A região em branco são os casos em que os indivíduos ficam fora do mercado, por insuficiência de renda ou de patrimônio.

Como as variáveis aleatórias $\boldsymbol{Y}^{(j)}$ e $\boldsymbol{W}^{(j)}$ têm distribuição conjunta $f_{Y, W}(y, w)$, calculamos $\mathbb{P}\left(\boldsymbol{J}_{t}^{(j)}=1 \mid \boldsymbol{P}_{t-1}\right)$ integrando $f_{Y, W}(y, w)$ sobre a região cinza da Figura 2.2.1. Assim, a probabilidade em (2.2.8) é dada por:

$$
\begin{aligned}
\mathbb{P}\left(\boldsymbol{J}_{t}^{(j)}=1 \mid \boldsymbol{P}_{t-1}\right)=\int_{(1-R) b \boldsymbol{P}_{t-1}}^{b \boldsymbol{P}_{t-1}} \int_{k\left(b \boldsymbol{P}_{t-1}-w\right)}^{\infty} f_{Y, W}(y, w) d y d w+ \\
+\int_{b \boldsymbol{P}_{t-1}}^{\infty} \int_{0}^{\infty} f_{Y, W}(y, w) d y d w .
\end{aligned}
$$

Note que $\mathbb{P}\left(\boldsymbol{J}_{t}^{(j)}=1 \mid \boldsymbol{P}_{t-1}\right)$ é função de $\boldsymbol{P}_{t-1}$, do parâmetro $b$ e também dos parâmetros $k$ e $R$, que dependem das regras de concessão de financiamento vigentes. Vamos então definir a função $\mathfrak{g}\left(\boldsymbol{P}_{t-1} ; b, k, R\right):=\mathbb{P}\left(\boldsymbol{J}_{t}^{(j)}=1 \mid \boldsymbol{P}_{t-1}\right)$ :

Definição 1. A probabilidade de o comprador $j$ obter os recursos para entrar no mercado no instante $t$, dado o preço de mercado no instante anterior, é dada pela função $\mathfrak{g}\left(\boldsymbol{P}_{t-1} ; b, k, R\right)$, calculada conforme (2.2.9).

Fato 2. Partindo da definição da variável aleatória $\boldsymbol{J}_{t}^{(j)}$ dada em (2.2.2), sabemos que $\boldsymbol{J}_{t}^{(j)} \mid$ $\boldsymbol{P}_{t-1}$ tem distribuição de Bernoulli com probabilidade de sucesso igual a $\mathfrak{g}\left(\boldsymbol{P}_{t-1} ; b, k, R\right)$ :

$$
\boldsymbol{J}_{t}^{(j)} \mid \boldsymbol{P}_{t-1} \sim \operatorname{Bernoulli}\left(\mathfrak{g}\left(\boldsymbol{P}_{t-1} ; b, k, R\right)\right) .
$$


A Figura 2.2.1 permite analisar graficamente o que acontece com a probabilidade de sucesso $\mathfrak{g}\left(\boldsymbol{P}_{t-1} ; b, k, R\right)$ quando o preço ou os parâmetros do SFH variam, e assim verificar a consistência construtiva do modelo. Note que:

1. Quando $\boldsymbol{P}_{t-1}$ aumenta, a área das Regiões I e II diminui. Portanto, $\mathfrak{g}\left(\boldsymbol{P}_{t-1} ; b, k, R\right)$ é não-crescente em relação a $\boldsymbol{P}_{t-1}$. De fato, com o aumento dos preços, menos compradores terão renda ou patrimônio suficientes para entrar no mercado.

2. Se $R$ aumenta, a área da Região I aumenta. Assim, $\mathfrak{g}\left(\boldsymbol{P}_{t-1} ; b, k, R\right)$ é não-decrescente em relação a $R$. Lembramos que, pela condição (2.2.5), um aumento em $R$ corresponde a um relaxamento nas regras de concessão de financiamento na forma de um LTV máximo maior, de forma que mais compradores terão condições de entrar no mercado.

3. Quando o parâmetro $k$ aumenta, a área da Região I diminui, ou seja, $\mathfrak{g}\left(\boldsymbol{P}_{t-1} ; b, k, R\right)$ é não-crescente em relação a $k$. Pela condição (2.2.6), um aumento em $k$ corresponde a um aumento das restrições na concessão de financiamento, na forma de aumento na taxa de juros $r$, redução no prazo máximo de financiamento $\tau$ ou redução no percentual de comprometimento de renda máximo $\rho$. Neste caso, como esperado, menos compradores conseguirão entrar no mercado.

\subsubsection{Compradores potenciais}

Como ficará claro na Seção 2.5, para calcular a função de demanda relativa e para obter a distribuição do preço de mercado, será necessário conhecer a distribuição do número de compradores potenciais no instante $t$. Os compradores potenciais são todos aqueles que conseguiram recursos para entrar no mercado no instante $t$. Considere, então, a seguinte definição:

Definição 3. Um comprador $j$ é considerado um comprador potencial no instante $t$ quando consegue obter os recursos para entrar no mercado no instante $t$, ou seja, quando $\boldsymbol{J}_{t}^{(j)}=1$.

Usando esta definição, o número de compradores potenciais no instante $t$ é dado por

$$
\boldsymbol{N}_{t}^{(B)}=\sum_{j=1}^{C} \boldsymbol{J}_{t}^{(j)}
$$

Para saber qual é a distribuição (condicionada ao passado) do número de compradores potenciais, observe que, do Fato 2, a variável $\boldsymbol{J}_{t}^{(j)}$ condicionada ao preço no instante anterior tem 
distribuição de Bernoulli com probabilidade de sucesso $\mathfrak{g}\left(\boldsymbol{P}_{t-1} ; b, k, R\right)$. Além disso, pela Hipótese 5, os vetores aleatórios $\left(\boldsymbol{Y}^{(j)}, \boldsymbol{W}^{(j)}\right), j=1, \ldots, C$, são independentes. Como a probabilidade de sucesso $\mathfrak{g}\left(\boldsymbol{P}_{t-1} ; b, k, R\right)$ depende apenas de $\boldsymbol{P}_{t-1}$ e da distribuição de $\left(\boldsymbol{Y}^{(j)}, \boldsymbol{W}^{(j)}\right)$, sabemos então que:

Fato 4. As variáveis $\boldsymbol{J}_{t}^{(1)}, \ldots, \boldsymbol{J}_{t}^{(C)}$, são condicionalmente independentes dado $\boldsymbol{P}_{t-1}$.

Com este fato, temos que o número de compradores potenciais condicionado ao passado é uma soma de $C$ variáveis aleatórias com distribuição de Bernoulli com probabilidade de sucesso $\mathfrak{g}\left(\boldsymbol{P}_{t-1} ; b, k, R\right)$. Assim, temos que:

Fato 5. A distribuição condicional do número de compradores potenciais é

$$
\boldsymbol{N}_{t}^{(B)} \mid \boldsymbol{P}_{t-1} \sim \operatorname{Binomial}\left(C, \mathfrak{g}\left(\boldsymbol{P}_{t-1} ; b, k, R\right)\right)
$$

Então, a esperança e a variância de $\boldsymbol{N}_{t}^{(B)} \mid \boldsymbol{P}_{t-1}$ são dadas por

$$
\begin{gathered}
\mu_{B}(t):=\mathrm{E}\left[\boldsymbol{N}_{t}^{(B)} \mid \boldsymbol{P}_{t-1}\right]=C \mathfrak{g}\left(\boldsymbol{P}_{t-1} ; b, k, R\right), \\
\sigma_{B}^{2}(t):=\operatorname{Var}\left(\boldsymbol{N}_{t}^{(B)} \mid \boldsymbol{P}_{t-1}\right)=C \mathfrak{g}\left(\boldsymbol{P}_{t-1} ; b, k, R\right)\left(1-\mathfrak{g}\left(\boldsymbol{P}_{t-1} ; b, k, R\right)\right) .
\end{gathered}
$$

\subsection{Vendedores}

Os vendedores anunciam seus imóveis por um valor que depende da sua pressa, e também do preço de mercado no instante anterior e da função de demanda relativa também no instante anterior. Enquanto a pressa é característica idiossincrática de cada vendedor, o preço de mercado e a função de demanda relativa estão disponíveis a todos os agentes.

Há $V$ vendedores no mercado. No instante de tempo $t \in \mathbb{N}$, cada vendedor $i, i=1, \ldots, V$, anuncia seu imóvel por

$$
\boldsymbol{A}_{t}^{(i)}=\boldsymbol{a}^{(i)} \boldsymbol{P}_{t-1} \Delta_{t-1}
$$

em que $\boldsymbol{P}_{t-1}$ é o preço de mercado apurado com base nas negociações em $t-1$, conforme definido em (2.1.1), $\boldsymbol{a}^{(i)}$ é uma variável aleatória não negativa com função de distribuição 


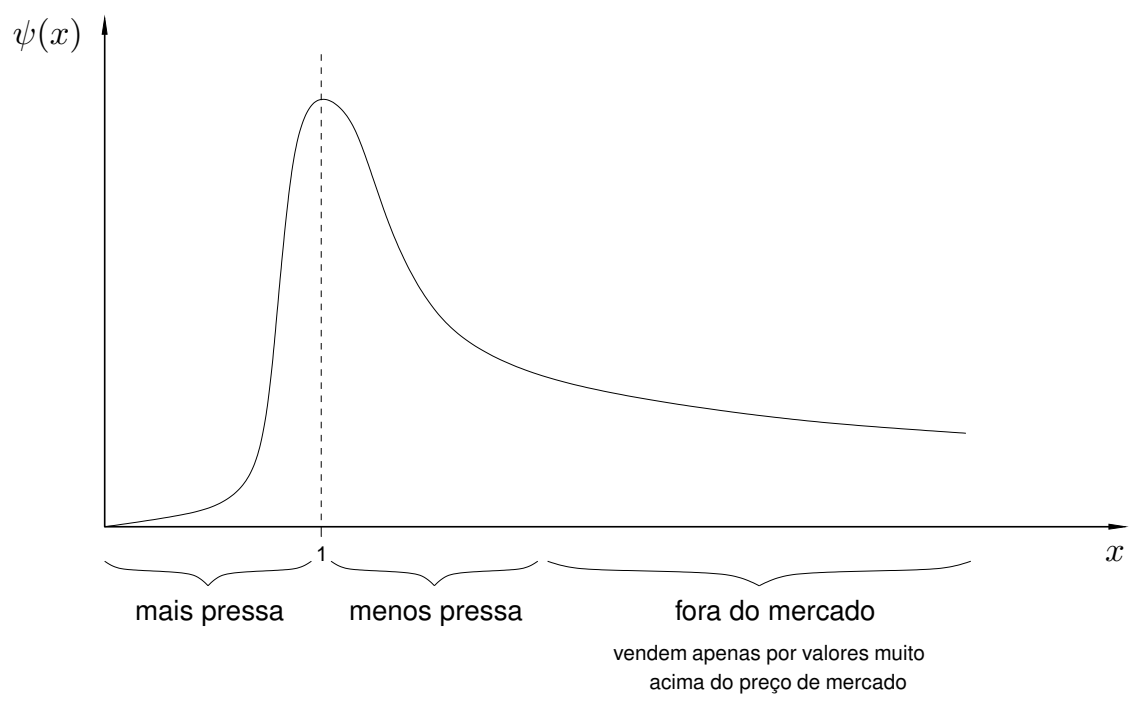

Figura 2.3.1: Densidade hipotética de $\boldsymbol{a}^{(i)}$.

$\Psi$, e $\Delta_{t}$ é uma função de demanda relativa que será definida posteriormente. Assim como o preço de mercado apurado no passado, o valor da função de demanda relativa apurada no passado também está disponível aos vendedores no instante $t$.

A variável aleatória $\boldsymbol{a}^{(i)}$, cuja distribuição é $\Psi$, representa a pressa do vendedor em vender o imóvel. Se o vendedor estiver disposto a vender o imóvel por menos que o preço de mercado disponível, i.e., tem mais pressa em realizar a venda, então $\boldsymbol{a}^{(i)}<1$. Se estiver disposto a vender apenas por um valor maior que o preço de mercado, i.e., tem menos pressa, então $\boldsymbol{a}^{(i)}>$ 1. A Figura 2.3.1 ilustra essas situações sobre uma densidade hipotética para $\boldsymbol{a}^{(i)}$. O mesmo conceito foi usado por Silva [16] para modelar o comportamento dos agentes na dinâmica de um livro de ofertas da bolsa (ver Seção 1.1.2).

\subsubsection{Distribuição do valor dos anúncios}

Lembramos que, pela Hipótese 4, os agentes só têm disponível no instante $t$ informações sobre o preço de mercado no passado e sobre a função de demanda relativa no passado. No caso dos vendedores, a equação (2.3.1) define que esses agentes utilizam tanto o preço de mercado como a função de demanda relativa no instante imediatamente anterior. Para simplificar a notação, usaremos a seguinte definição:

Definição 6. As informações disponíveis aos agentes vendedores no instante $t+1$ são o vetor aleatório $\left[\boldsymbol{P}_{t}, \Delta_{t}\right]$.

Vamos agora calcular a distribuição condicional de $\boldsymbol{A}_{t}^{(i)} \mid \boldsymbol{P}_{t-1}, \Delta_{t-1}$ : 


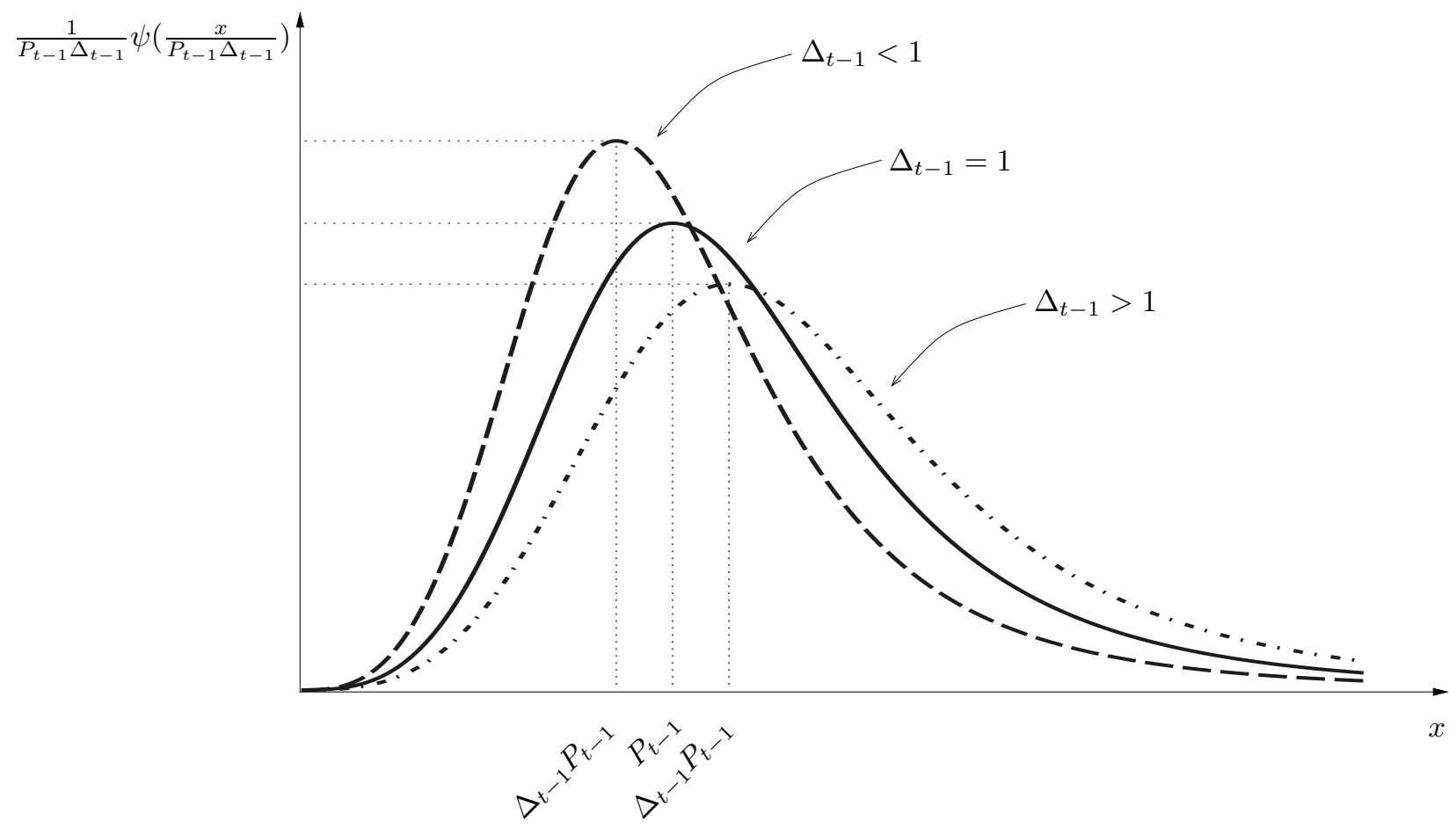

Figura 2.3.2: Densidade hipotética de $\boldsymbol{A}_{t}^{(i)}$ quando a liquidez aumenta e diminui.

Fato 7. A distribuição condicional de $\boldsymbol{A}_{t}^{(i)} \mid \boldsymbol{P}_{t-1}, \Delta_{t-1} e^{\prime}$

$$
\begin{aligned}
\mathbb{P}\left(\boldsymbol{A}_{t}^{(i)} \leq x \mid \boldsymbol{P}_{t-1}, \Delta_{t-1}\right) & =\mathbb{P}\left(\boldsymbol{a}^{(i)} \boldsymbol{P}_{t-1} \Delta_{t-1} \leq x \mid \boldsymbol{P}_{t-1}, \Delta_{t-1}\right) \\
& =\mathbb{P}\left(\boldsymbol{a}^{(i)} \leq \frac{x}{\boldsymbol{P}_{t-1} \Delta_{t-1}} \mid \boldsymbol{P}_{t-1}, \Delta_{t-1}\right) \\
& =\Psi\left(\frac{x}{\boldsymbol{P}_{t-1} \Delta_{t-1}}\right) .
\end{aligned}
$$

A Figura 2.3.2 mostra o que ocorre com a densidade de $\boldsymbol{A}_{t}^{(i)}$ quando a função de demanda relativa aumenta e quando diminui. Quando $\Delta_{t-1}>1$ (maior demanda relativa), então a massa em valores mais altos de $\boldsymbol{A}_{t}^{(i)}$ fica maior, ou seja, os vendedores passam a anunciar seus imóveis por valores maiores. Por outro lado, quando $\Delta_{t-1}<1$ (menor demanda relativa), a massa em valores mais altos de $\boldsymbol{A}_{t}^{(i)}$ fica menor, ou seja, os vendedores passam a anunciar seus imóveis por valores menores.

\subsubsection{Vendedores potenciais}

Assim como foi feito na Seção 2.2 para os agentes compradores, vamos agora definir quem e quantos são os vendedores potenciais. Isso será importante para definir a função demanda relativa e para calcular a distribuição condicional do preço de mercado. Para isso, lembramos que os compradores têm um preço de reserva de $b \boldsymbol{P}_{t-1}$. A existência de um preço de reserva 
dos compradores significa que, no instante $t$, estes agentes não aceitam pagar mais do que $b \boldsymbol{P}_{t-1}$ por um imóvel. Assim, num dado instante $t$, um vendedor que anuncie por mais do que $b \boldsymbol{P}_{t-1}$ certamente não conseguirá vender o seu imóvel, pois não há qualquer comprador disposto a pagar o preço por ele anunciado. Já o vendedor que anuncia o seu imóvel por valor menor ou igual ao preço de reserva dos compradores pode, potencialmente, realizar uma venda. Esta constatação nos leva à seguinte definição:

Definição 8. Um vendedor $i$ é considerado um vendedor potencial no instante $t$ quando pode, potencialmente, realizar uma venda, ou seja, quando $\boldsymbol{A}_{t}^{(i)} \leq b \boldsymbol{P}_{t-1}$. De forma equivalente, definimos a variável indicadora

$$
\boldsymbol{I}_{t}^{(i)}= \begin{cases}1, & \text { se } \boldsymbol{A}_{t}^{(i)} \leq b \boldsymbol{P}_{t-1} \\ 0, & \text { c.c. }\end{cases}
$$

e o vendedor $i$ é considerado um vendedor potencial se, e somente se, $\boldsymbol{I}_{t}^{(i)}=1$.

Já sabemos quem são os vendedores potenciais. Agora, vamos responder a outra questão: quantos são os vendedores potenciais? Para isso, precisaremos calcular a probabilidade de o vendedor $i$ ser um vendedor potencial. Usando o Fato 7, temos:

Fato 9. A probabilidade condicional (ao passado) de o vendedor $i$ ser um vendedor potencial é

$$
\begin{aligned}
\mathbb{P}\left(\boldsymbol{I}_{t}^{(i)}=1 \mid \boldsymbol{P}_{t-1}, \Delta_{t-1}\right) & =\mathbb{P}\left(\boldsymbol{A}_{t}^{(i)} \leq b \boldsymbol{P}_{t-1} \mid \boldsymbol{P}_{t-1}, \Delta_{t-1}\right) \\
& =\Psi\left(\frac{b \boldsymbol{P}_{t-1}}{\Delta_{t-1} \boldsymbol{P}_{t-1}}\right) \\
& =\Psi\left(\frac{b}{\Delta_{t-1}}\right)
\end{aligned}
$$

ou, em outras palavras,

$$
\boldsymbol{I}_{t}^{(i)} \mid \boldsymbol{P}_{t-1}, \Delta_{t-1} \sim \operatorname{Bernoulli}\left(\Psi\left(\frac{b}{\Delta_{t-1}}\right)\right) .
$$

Como $\boldsymbol{I}_{t}^{(i)}=1$ se, e somente se, $i$ é um vendedor potencial, então o número vendedores potenciais no instante $t$ é 


$$
\boldsymbol{N}_{t}^{(A)}=\sum_{i=1}^{V} \boldsymbol{I}_{t}^{(i)}
$$

Lembramos que, pela Hipótese 4, no instante $t$ os agentes têm informação apenas sobre o preço de mercado passado e sobre a função de demanda relativa passada, mas não têm informação sobre as decisões dos demais agentes no próprio instante $t$. Assim, as variáveis aleatórias $\boldsymbol{I}_{t}^{(1)}, \ldots, \boldsymbol{I}_{t}^{(V)}$ não são independentes, pois os vendedores utilizam, no instante $t$, informações sobre as decisões dos demais agentes no passado, disponíveis a cada um de forma agregada por meio das variáveis $\boldsymbol{P}_{t-1}$ e $\Delta_{t-1}$. Mas, condicionado ao passado, temos o seguinte:

Fato 10. As variáveis $\boldsymbol{I}_{t}^{(1)}, \ldots, \boldsymbol{I}_{t}^{(V)}$ são condicionalmente independentes dado $\Delta_{t-1}$.

Usando os Fatos 9 e 10, sabemos que o número de vendedores potenciais, condicionado ao passado, é uma soma de $V$ variáveis aleatórias independentes com distribuição de Bernoulli com probabilidade de sucesso $\Psi\left(\frac{b}{\Delta_{t-1}}\right)$. Assim, temos o seguinte:

Fato 11. A distribuição condicional do número de vendedores potenciais é

$$
\boldsymbol{N}_{t}^{(A)} \mid \Delta_{t-1} \sim \operatorname{Binomial}\left(V, \Psi\left(\frac{b}{\Delta_{t-1}}\right)\right)
$$

Então, a esperança e a variância de $\boldsymbol{N}_{t}^{(A)} \mid \Delta_{t-1}$ são dadas por

$$
\begin{gathered}
\mu_{A}(t):=\mathrm{E}\left[\boldsymbol{N}_{t}^{(A)} \mid \Delta_{t-1}\right]=V \Psi\left(\frac{b}{\Delta_{t-1}}\right), \\
\sigma_{A}^{2}(t):=\operatorname{Var}\left(\boldsymbol{N}_{t}^{(A)} \mid \Delta_{t-1}\right)=V \Psi\left(\frac{b}{\Delta_{t-1}}\right)\left(1-\Psi\left(\frac{b}{\Delta_{t-1}}\right)\right) .
\end{gathered}
$$

\subsection{Função de demanda relativa $\Delta_{t}$}

A função de demanda relativa é fundamental para o modelo. Junto com o preço de mercado, ela é a informação resumida sobre o passado que os vendedores utilizam para modificar o seu comportamento em função de mudanças ocorridas no mercado. Ela representa a percepção que os vendedores têm sobre o mercado: seu valor é maior quando o mercado está num "bom" momento e é menor quando está num "mau" momento. 
Lembramos que o vendedor $i$ anuncia seu imóvel por $\boldsymbol{A}_{t}^{(i)}=\boldsymbol{a}^{(i)} \boldsymbol{P}_{t-1} \Delta_{t-1}$. Assim, a função de demanda relativa deve ser definida de forma a ser sempre maior que 0 , já que um anúncio por valor negativo não tem significado na realidade. Além disso, para que os vendedores respondam corretamente a mudanças na demanda, ela deve ser e tal que, quando não ocorrem mudanças, seu valor seja igual a 1 ; quando a demanda diminui, deve ser menor que 1 ; e, quando a demanda aumenta, deve ser maior que 1.

Também é desejável que a função de demanda relativa dependa também do passado, de forma a representar a memória de longo prazo dos vendedores sem perder a característica markoviana do processo.

Considerando essas características, utilizaremos o seguinte:

Definição 12. Definimos a função de demanda relativa como

$$
\Delta_{t}:=\left(\Delta_{t-1}\right)^{\alpha} \exp \left[\frac{\lambda}{C+V}\left(\boldsymbol{N}_{t}^{(B)}-\boldsymbol{N}_{t}^{(A)}\right)\right]
$$

com $\alpha \in[0,1)$ o parâmetro de memória dos vendedores e $\lambda>0$ o parâmetro de sensibilidade à demanda dos vendedores.

A memória dos vendedores é representada por $\alpha \in[0,1)$. Note que, quando $\alpha=0$, os vendedores têm memória apenas do instante imediatamente anterior, i.e., ao definir o preço do anúncio no instante $t$, consideram apenas a diferença entre compradores e vendedores potenciais ocorrida em $t-1$. Se $\alpha>0$, então os vendedores consideram também essa diferença no passado mais distante, embora com peso menor do que a do passado recente.

O parâmetro $\lambda>0$ representa a sensibilidade dos vendedores variações na demanda: quanto maior o seu valor, mais rápida é a resposta dos vendedores ao excesso ou escassez de demanda observado. Esse parâmetro pode suavizar ou amplificar o efeito que a variação na demanda tem sobre os valores dos anúncios dos vendedores: quando $\lambda>1$, o efeito do excesso de demanda sobre a variável aleatória $\boldsymbol{A}_{t}^{(i)}$ será amplificado; e quando $0<\lambda<1$, esse efeito é suavizado. Assim, quanto maior for o valor de $\lambda$, mais intensamente os vendedores ajustam seus anúncios (para valores maiores) em resposta a aumentos na demanda.

\subsubsection{Rigidez dos preços na tendência de queda}

É interessante lembrar que um dos fatos estilizados observados no mercado imobiliário é o de que os preços apresentam rigidez para baixo, resultando em aumento da liquidez quando 
há tendência de aumento nos preços e de redução da liquidez quando há tendência de diminuição dos preços $[10,9]$. Ao definir a função de demanda relativa como uma exponencial da diferença entre o número de compradores e o número de vendedores, como em (2.4.1), obtemos um comportamento dos vendedores mais sensível a aumentos do que a reduções na demanda. Isso acontece porque a função

$$
\frac{d}{d x}\left(e^{\lambda x}\right)=\lambda e^{x}
$$

é crescente em $x$, o que significa que, quando o número de compradores potenciais diminui, a mudança no valor de $\Delta_{t}$ é mais fraca do que quando o número de compradores potenciais aumenta. Como os vendedores alteram os valores dos seus anúncios em função de $\Delta_{t}$, eles responderão mais lentamente a uma redução do que a aumento na demanda.

\subsection{Número e valor das transações}

Nas Seções 2.2 e 2.3, vimos como se comportam os compradores e os vendedores, e como esse comportamento determina o número de compradores e de vendedores potenciais e a distribuição do valor dos anúncios em cada instante. Em seguida, vimos na Seção 2.4 como é definida a função de demanda relativa, que é a variável usada pelos vendedores para ajustar o valor dos seus anúncios.

Nesta seção, vamos obter a distribuição (condicionada ao passado) do valor das transações $\boldsymbol{T}_{t}^{(k)}$ ocorridas no instante $t$, e do número de transações $\boldsymbol{n}_{t}$. Lembre-se de que o preço de mercado no instante $t$ é dado por $\boldsymbol{P}_{t}=\frac{1}{\boldsymbol{n}_{t}} \sum_{k=1}^{\boldsymbol{n}_{t}} \boldsymbol{T}_{t}^{(k)}$, ou seja, ele é a média das transações ocorridas naquele instante. Assim, para saber qual será a dinâmica dos preços, precisamos então obter a distribuição do valor das transações $\boldsymbol{T}_{t}^{(k)}$ e do número de transações $\boldsymbol{n}_{t}$, o que será feito nas seções 2.5.1 e 2.5.2. A distribuição do preço não pode ser calculada de forma explícita mas, no Capítulo 3, obteremos um resultado que nos permitirá analisar a dinâmica do preço mesmo sem obter a sua distribuição.

\subsubsection{Número de transações}

Nesta seção, vamos obter a distribuição do número de transações que ocorrem no instante $t$.

A cada instante, os vendedores anunciam seus imóveis nas condições descritas na Seção 2.3 e os compradores tentam obter recursos para entrar no mercado, nas condições descritas na Seção 2.2. Já definimos que os compradores que conseguem os recursos para entrar no 
mercado são os compradores potenciais, e os vendedores que anunciam por menos que o preço de reserva dos compradores são os vendedores potenciais. Num dado instante $t$, há $\boldsymbol{N}_{t}^{(B)}$ compradores potenciais e $\boldsymbol{N}_{t}^{(A)}$ vendedores potenciais, e já mostramos nos Fatos 5 e 11 que as suas distribuições condicionais são

$$
\begin{gathered}
\boldsymbol{N}_{t}^{(B)} \mid \boldsymbol{P}_{t-1} \sim \operatorname{Binomial}\left(C, \mathfrak{g}\left(\boldsymbol{P}_{t-1} ; b, k, R\right)\right), \\
\boldsymbol{N}_{t}^{(A)} \mid \Delta_{t-1} \sim \operatorname{Binomial}\left(V, \Psi\left(\frac{b}{\Delta_{t-1}}\right)\right) .
\end{gathered}
$$

Lembramos que uma transação ocorre quando um comprador que entrou no mercado, i.e., um comprador potencial, encontra um vendedor que anunciou por menos que o seu preço de reserva, i.e., um vendedor potencial. Num dado instante de tempo $t$, pode ocorrer uma de duas situações:

1. $\boldsymbol{N}_{t}^{(A)}>\boldsymbol{N}_{t}^{(B)}$ : há mais vendedores potenciais do que compradores potenciais. Neste caso, todos os compradores que entraram no mercado conseguirão fechar uma transação, ou seja, o número de transações será $\boldsymbol{n}_{t}=\boldsymbol{N}_{t}^{(B)}$; ou

2. $\boldsymbol{N}_{t}^{(A)}<\boldsymbol{N}_{t}^{(B)}$ : há menos vendedores potenciais do que compradores potenciais. Nesta situação, alguns compradores com os recursos para comprar não poderão fazê-lo, mas todos os vendedores potenciais farão a venda. Neste caso, o número de transações será $\boldsymbol{n}_{t}=\boldsymbol{N}_{t}^{(A)}$.

Assim,

$$
\boldsymbol{n}_{t}=\min \left\{\boldsymbol{N}_{t}^{(A)}, \boldsymbol{N}_{t}^{(B)}\right\}
$$

Na seção 3.3, vamos precisar da expressão que nos dá a distribuição de $\boldsymbol{n}_{t}$ para obter a dinâmica dos preços de mercado. Antes de calcular essa distribuição, porém, vamos introduzir a seguinte notação para a probabilidade condicionada ao passado:

$$
\mathbb{P}_{t-1}(\cdot):=\mathbb{P}\left(\cdot \mid \boldsymbol{P}_{t-1}, \Delta_{t-1}\right)
$$

A distribuição de $\boldsymbol{n}_{t}$ (condicionada ao passado), que é o mínimo entre duas binomiais condicionalmente independentes, pode ser representada pela seguinte expressão: 
Fato 13. A distribuição condicional de $\boldsymbol{n}_{t}$ é dada por

$$
\begin{aligned}
& \mathbb{P}_{t-1}\left(\boldsymbol{n}_{t}=n\right)=\mathbb{P}_{t-1}(\left.\boldsymbol{N}_{t}^{(A)}=n\right)+\mathbb{P}_{t-1}\left(\boldsymbol{N}_{t}^{(B)}=n\right) \\
&-\mathbb{P}_{t-1}\left(\boldsymbol{N}_{t}^{(A)}=n\right) \mathbb{P}_{t-1}\left(\boldsymbol{N}_{t}^{(B)} \leq n\right) \\
& \quad-\mathbb{P}_{t-1}\left(\boldsymbol{N}_{t}^{(B)}=n\right) \mathbb{P}_{t-1}\left(\boldsymbol{N}_{t}^{(A)} \leq n-1\right)
\end{aligned}
$$

Demonstração. Ver apêndice B.1.

\subsubsection{Valor das transações}

Na seção anterior, obtivemos a distribuição (condicionada ao passado) do número de transações que ocorrem no instante $t$. Agora, vamos calcular a distribuição do valor das transações. Mas, para fazer isso, precisaremos de uma nova hipótese:

Hipótese 7. A distribuição da pressa dos vendedores é não nula para valores positivos de $\boldsymbol{a}^{(i)}$ ou seja, $\Psi(x)>0 \forall x>0$.

Esta hipótese garantirá que, mesmo para valores grandes de $\Delta_{t-1}$, a probabilidade de haver algum vendedor potencial no instante $t$ é positiva, como fica claro na seguinte observação:

Observação 14. Note que, no instante $t$, o número de vendedores potenciais $\boldsymbol{N}_{t}^{(A)} \mid \Delta_{t-1}$ é uma binomial com probabilidade de sucesso igual a $\Psi\left(\frac{b}{\Delta_{t-1}}\right)$. Assim, como $\Psi$ é não decrescente, a não observância da Hipótese 7 significa que existe um $\Delta^{(g)}$ tal que, $\forall \Delta_{t-1} \geq \Delta^{(g)}$, o número de vendedores potenciais em $t$ será uma binomial com probabilidade de sucesso nula, o que corresponde a zero vendedores potenciais com probabilidade 1 no instante $t$; e, além disso, que o número de transações, $\boldsymbol{n}_{t}=\min \left\{\boldsymbol{N}_{t}^{(A)}, \boldsymbol{N}_{t}^{(B)}\right\}$, também será zero com probabilidade 1 .

Para obter a distribuição do valor das transações, lembramos que $\boldsymbol{T}_{t}^{(k)}$ é o valor da $k$-ésima transação fechada em $t$, e que as transações são fechadas pelo valor anunciado pelo vendedor, $\boldsymbol{A}_{t}^{(i)}$, e não pelo preço de reserva do comprador. Apenas os vendedores que anunciam por valores menores ou iguais a $b \boldsymbol{P}_{t-1}$ podem potencialmente ser escolhidos por um comprador para fechar a transação, i.e., são vendedores potenciais. Os demais vendedores estão fora do mercado, no sentido de que nenhum comprador tentará comprar o seu imóvel no instante $t$. Assim, os valores $\boldsymbol{T}_{t}^{(k)}$ das transações serão sorteados dentre os valores de $\boldsymbol{A}_{t}^{(i)}, i=1, \ldots, V$, tais que $\boldsymbol{A}_{t}^{(i)} \leq b \boldsymbol{P}_{t-1}$, conforme a Figura 2.5.1.

Observe, na Figura 2.5.1, que os valores de $\boldsymbol{T}_{t}^{(k)}$ são sorteados apenas da região cinza da curva. Da cauda da curva, correspondente aos anúncios maiores que o preço de reserva dos 


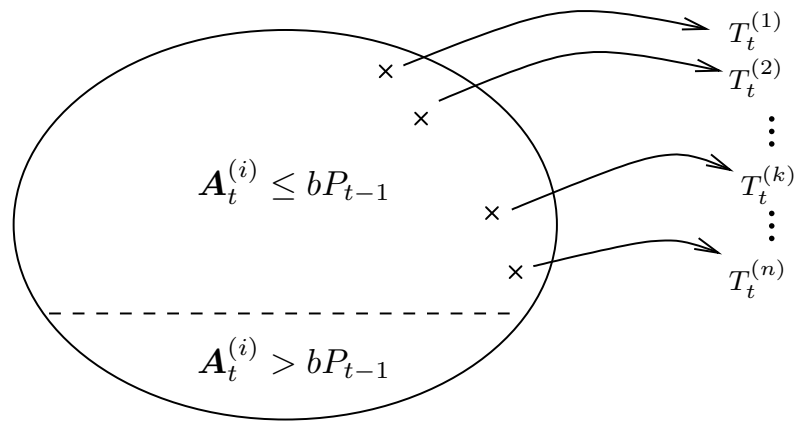

(a)

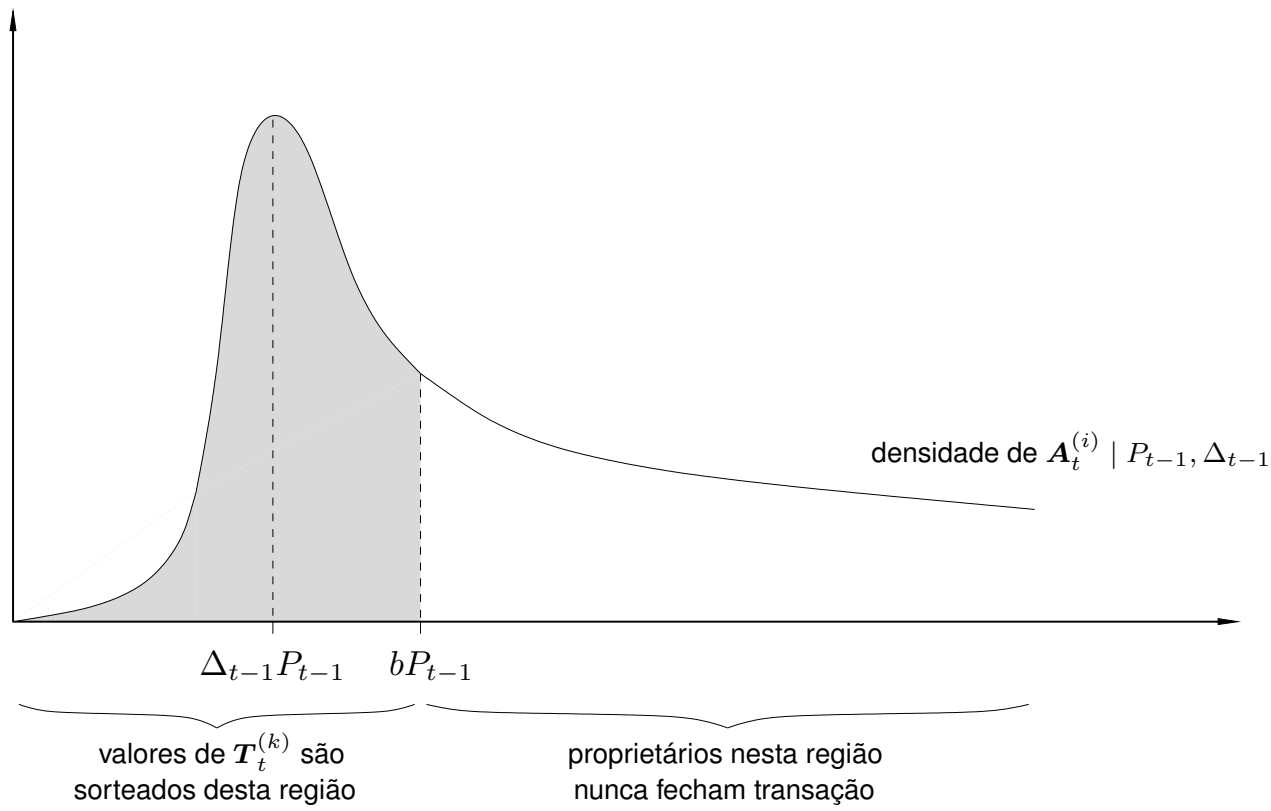

(b)

Figura 2.5.1: Valores das transações, $\boldsymbol{T}_{t}^{(k)}$. 
compradores, não serão sorteados quaisquer valores de transação, porque os compradores não aceitam pagar mais do que o seu preço de reserva. Assim, fica claro que a densidade dos valores de $\boldsymbol{T}_{t}^{(k)}$ será apenas a região cinza, excluída a cauda, e normalizada pela sua área.

Então, a distribuição condicional de $\boldsymbol{T}_{t}^{(k)}$ é dada pela distribuição condicional

$$
\begin{aligned}
\mathbb{P}\left(\boldsymbol{T}_{t}^{(k)} \leq x \mid \boldsymbol{P}_{t-1}, \Delta_{t-1}\right) & =\mathbb{P}_{t-1}\left(\boldsymbol{A}_{t}^{(i)} \leq x \mid \boldsymbol{A}_{t}^{(i)} \leq b \boldsymbol{P}_{t-1}\right) \\
& =\frac{\mathbb{P}_{t-1}\left(\boldsymbol{A}_{t}^{(i)} \leq x \text { e } \boldsymbol{A}_{t}^{(i)} \leq b \boldsymbol{P}_{t-1}\right)}{\mathbb{P}_{t-1}\left(\boldsymbol{A}_{t}^{(i)} \leq b \boldsymbol{P}_{t-1}\right)}
\end{aligned}
$$

Note que, para que a distribuição dada por (2.5.3) esteja bem definida, o seu denominador deve ser diferente de zero. Lembramos que a distribuição condicional de $\boldsymbol{A}_{t}^{(i)}$ é dada por (2.3.2) e, portanto,

$$
\mathbb{P}_{t-1}\left(\boldsymbol{A}_{t}^{(i)} \leq b \boldsymbol{P}_{t-1}\right)=\Psi\left(\frac{b}{\Delta_{t-1}}\right)
$$

Assim, a Hipótese 7 garante que este denominador é maior que zero qualquer que seja o valor de $\Delta_{t-1}$ e, portanto, a distribuição de $\boldsymbol{T}_{t}^{(k)}$ está bem definida, e pode ser calculada.

Proposição 15. Sob a Hipótese 7, a distribuição (condicionada ao passado) de $\boldsymbol{T}_{t}^{(k)}$ é dada por

$$
\mathbb{P}\left(\boldsymbol{T}_{t}^{(k)} \leq x \mid \boldsymbol{P}_{t-1}, \Delta_{t-1}\right)= \begin{cases}1, & x>b \boldsymbol{P}_{t-1} \\ \frac{\Psi\left(\frac{x}{\boldsymbol{P}_{t-1} \Delta_{t-1}}\right)}{\Psi\left(\frac{b}{\Delta_{t-1}}\right)}, & x \leq b \boldsymbol{P}_{t-1}\end{cases}
$$

Demonstração. Ver apêndice B.2.

A Figura 2.5.2 ilustra uma distribuição de $\boldsymbol{A}_{t}^{(i)}$ e a distribuição de $\boldsymbol{T}_{t}^{(k)}$ resultante. Observe que a curva da distribuição de $\boldsymbol{T}_{t}^{(k)}$ é uma versão "achatada", na direção horizontal, da curva da distribuição de $\boldsymbol{A}_{t}^{(i)}$, de forma a chegar a 1 quando $x=b \boldsymbol{P}_{t-1}$.

Um resultado importante sobre a variável $\boldsymbol{T}_{t}^{(k)}$ que será usado mais adiante é o seguinte:

Corolário 16. A variável $\boldsymbol{T}_{t}^{(k)} \mid \boldsymbol{P}_{t-1}, \Delta_{t-1}$ tem todos os momentos finitos.

Demonstração. Observe que, pela distribuição dada em (2.5.4), o suporte da variável $\boldsymbol{T}_{t}^{(k)}$ $\boldsymbol{P}_{t-1}, \Delta_{t-1}$ é o intervalo finito $\left(0, b \boldsymbol{P}_{t-1}\right]$. Logo, todos os seus momentos são finitos. 


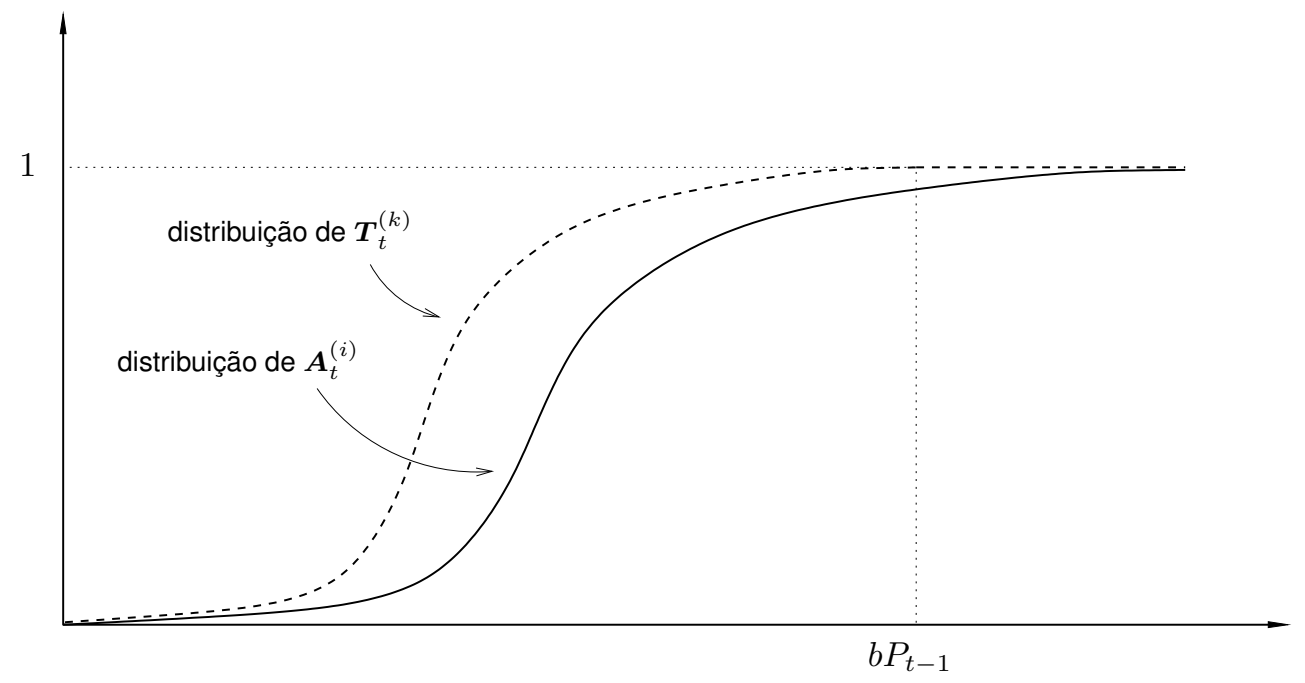

Figura 2.5.2: Distribuição de $\boldsymbol{A}_{t}^{(i)}$ e de $\boldsymbol{T}_{t}^{(k)}$. 


\section{Capítulo 3}

\section{Análise do modelo}

No Capítulo 2, definimos o modelo de agentes para o mercado imobiliário. Neste capítulo, vamos estudar o modelo e observar quais são as suas características emergentes. Para fazer isso, vamos primeiro mostrar que, quando o número de agentes aumenta, o processo estocástico $\left(\boldsymbol{P}_{t}, \Delta_{t}\right)$ converge para um sistema dinâmico determinístico. Isso será feito na Seção 3.2. Em seguida, conhecendo as equações do sistema dinâmico, podemos obter as condições de estabilidade e convergência do preço de mercado, e como ocorre essa convergência. Isso será feito na Seção 3.3.

A construção do modelo e a sua formulação foram apresentadas detalhadamente no Capítulo 2. Mas, para facilitar o entendimento a partir de agora, antes de prosseguir, vamos apresentar uma descrição resumida do processo na Seção 3.1 .

\subsection{Descrição resumida do modelo}

O modelo estudado neste trabalho é um processo de Markov a tempo discreto formado pelo $\operatorname{par}\left(\boldsymbol{P}_{t}, \Delta_{t}\right)$, em que $\boldsymbol{P}_{t}$ é o preço de mercado dos imóveis e $\Delta_{t}$ é uma função de demanda relativa, e cujo espaço de estados é o conjunto $\mathbb{R}_{+} \times \mathbb{R}_{+}$.

Dado o passado, $\boldsymbol{P}_{t}$ e $\Delta_{t}$ são independentes. Portanto, para descrever o processo, bastaria obter o kernel para ambos. Porém, o kernel para $\boldsymbol{P}_{t}$ e para $\Delta_{t}$ não podem ser obtidos explicitamente $^{1}$. Então, para descrever o processo, precisaremos usar algumas das equações da sua formulação, que apresentamos no Capítulo 2.

\footnotetext{
${ }^{1} \mathrm{O}$ kernel aproximado para $\Delta_{t}$ pode ser obtido. De fato, a distribuição da variável $\Delta_{t} \mid \boldsymbol{P}_{t-1}, \Delta_{t-1}$ converge para uma lognormal, mas este resultado não foi explicitamente usado no trabalho.
} 
O preço de mercado $\boldsymbol{P}_{t}$ é apurado como sendo a média das transações $\boldsymbol{T}_{t}^{(k)}$ :

$$
\boldsymbol{P}_{t}=\frac{1}{\boldsymbol{n}_{t}} \sum_{k=1}^{\boldsymbol{n}_{t}} \boldsymbol{T}_{t}^{(k)}
$$

O kernel de $\boldsymbol{T}_{t}^{(k)}$ é dado por

$$
\mathbb{P}_{t-1}\left(\boldsymbol{T}_{t}^{(k)} \leq x\right)= \begin{cases}1, & x>b \boldsymbol{P}_{t-1} \\ \frac{\Psi\left(\frac{x}{P_{t-1} \Delta_{t-1}}\right)}{\Psi\left(\frac{b}{\Delta_{t-1}}\right)}, & x \leq b \boldsymbol{P}_{t-1}\end{cases}
$$

O número de transações $\boldsymbol{n}_{t}$ é dado por $\boldsymbol{n}_{t}=\min \left\{\boldsymbol{N}_{t}^{(A)}, \boldsymbol{N}_{t}^{(B)}\right\}$, em que $\boldsymbol{N}_{t}^{(A)}$ é o número de vendedores potenciais e $\boldsymbol{N}_{t}^{(B)}$ é o número de compradores potenciais. O kernel de $\boldsymbol{N}_{t}^{(A)}$ e o kernel de $\boldsymbol{N}_{t}^{(B)}$ também são conhecidos, pois

$$
\boldsymbol{N}_{t}^{(A)} \mid \boldsymbol{P}_{t-1}, \Delta_{t-1} \sim \operatorname{Binomial}\left(V, \Psi\left(\frac{b}{\Delta_{t-1}}\right)\right)
$$

$\mathrm{e}$

$$
\boldsymbol{N}_{t}^{(B)} \mid \boldsymbol{P}_{t-1}, \Delta_{t-1} \sim \operatorname{Binomial}\left(C, \mathfrak{g}\left(\boldsymbol{P}_{t-1} ; b, k, R\right)\right),
$$

lembrando que $V$ e $C$ são o número de vendedores e de compradores, $b$ é o parâmetro que dá o preço de reserva dos compradores, $\Psi$ é a distribuição que descreve a pressa dos vendedores, $k$ e $R$ são parâmetros do SFH.

A função $\mathfrak{g}(\cdot)$ representa a probabilidade de um comprador conseguir recursos para entrar no mercado, e é dada por

$$
\mathfrak{g}\left(\boldsymbol{P}_{t} ; b, k, R\right)=\int_{(1-R) b \boldsymbol{P}_{t}}^{b \boldsymbol{P}_{t}} \int_{k\left(b \boldsymbol{P}_{t}-w\right)}^{\infty} f_{Y, W}(y, w) d y d w+\int_{b \boldsymbol{P}_{t}}^{\infty} \int_{0}^{\infty} f_{Y, W}(y, w) d y d w
$$

em que $f_{Y, W}(y, w)$ é a densidade conjunta de renda e riqueza, com suporte em $\mathbb{R}_{+} \times \mathbb{R}_{+}$.

Finalmente, a função de demanda relativa é definida como

$$
\Delta_{t}=\Delta_{t-1}^{\alpha} \exp \left[\frac{\lambda}{V+C}\left(\boldsymbol{N}_{t}^{(B)}-\boldsymbol{N}_{t}^{(A)}\right)\right] .
$$

\subsection{Convergência em número de agentes}

Nesta seção, vamos descobrir que o processo $\left(\boldsymbol{P}_{t}, \Delta_{t}\right)$ converge para um sistema dinâmico determinístico quando o número de agentes aumenta. Este resultado será obtido separada- 
mente para $\Delta_{t}$ e para $\boldsymbol{P}_{t}$ nas Seções 3.2.1 e 3.2.2. É possível separar os dois processos porque, pela Hipótese $4, \boldsymbol{P}_{t}$ e $\Delta_{t}$ são condicionalmente independentes dado o passado.

\subsubsection{Distribuição da função de demanda relativa}

A função de demanda relativa foi definida na Seção 2.4. Nesta seção, vamos obter o resultado da convergência da função de demanda relativa $\Delta_{t}$ a um valor determinístico $\delta_{t}$ quando o número de agentes aumenta, similar a uma Lei dos Grandes Números. Como ficará claro na Seção 3.3, este resultado nos possibilitará obter a dinâmica do preço e o preço de equilíbrio.

Ocorre que a função $\Delta_{t}$, como definida em (2.4.1), é um produto de variáveis aleatórias, enquanto que a função

$$
\log \Delta_{t}=\alpha \log \Delta_{t-1}+\frac{\lambda}{C+V}\left(\boldsymbol{N}_{t}^{(B)}-\boldsymbol{N}_{t}^{(A)}\right)
$$

é simplesmente uma soma, o que torna esta última representação mais conveniente. Assim, para obter a convergência de $\Delta_{t}$, vamos calcular a esperança e a variância de $\log \Delta_{t} \mid$ $\boldsymbol{P}_{t-1}, \Delta_{t-1}$. Em seguida, vamos obter a convergência de $\log \Delta_{t} \mid \boldsymbol{P}_{t-1}, \Delta_{t-1}$ quando o número de agentes aumenta.

Para simplificar a notação, utilizaremos a simplificação $\mathfrak{g}_{t-1}=\mathfrak{g}\left(\boldsymbol{P}_{t-1} ; b, k, R\right)$. Também vamos usar a seguinte definição:

Definição 17. A razão entre o número de vendedores e o número de compradores será denotada por

$$
\nu:=\frac{V}{C}
$$

Lema 18. A esperança e a variância condicionais da variável $\log \Delta_{t} \mid \boldsymbol{P}_{t-1}, \Delta_{t-1}$ são dadas por, respectivamente,

$$
\begin{gathered}
\mu_{t}=\alpha \log \Delta_{t-1}+\lambda\left(\frac{1}{1+\nu} \mathfrak{g}_{t-1}-\frac{\nu}{1+\nu} \Psi\left(\frac{b}{\Delta_{t-1}}\right)\right), \\
\sigma_{t}^{2}=\frac{\lambda^{2}}{V+C}\left[\frac{1}{1+\nu} \mathfrak{g}_{t-1}\left(1-\mathfrak{g}_{t-1}\right)+\frac{\nu}{1+\nu} \Psi\left(\frac{b}{\Delta_{t-1}}\right)\left(1-\Psi\left(\frac{b}{\Delta_{t-1}}\right)\right)\right] .
\end{gathered}
$$

Demonstração. Ver apêndice B.3.

Usando o resultado do Lema 18, podemos agora verificar que a variável $\log \Delta_{t} \mid \boldsymbol{P}_{t-1}, \Delta_{t-1}$ converge para o valor da sua esperança quando o número de agentes aumenta. Este resultado é apresentado no seguinte teorema: 
Teorema 19. Seja $n=V+C$ o número total de agentes e considere que a razão $V / C=\nu$ é constante. Então

$$
\log \Delta_{t} \mid \boldsymbol{P}_{t-1}, \Delta_{t-1} \underset{n \rightarrow \infty}{\stackrel{\mathbb{P}}{\longrightarrow}} \mu_{t}
$$

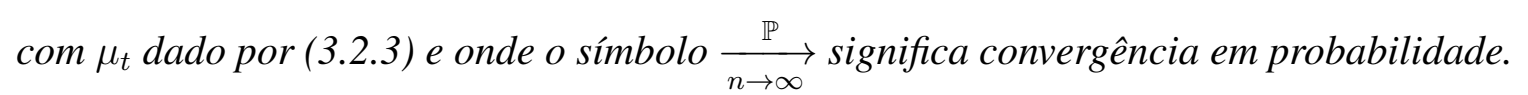

Demonstração. Lembrando que $\log \Delta_{t}=\alpha \log \Delta_{t-1}+\frac{\lambda}{C+V}\left(\boldsymbol{N}_{t}^{(B)}-\boldsymbol{N}_{t}^{(A)}\right)$, considere a sequência de variáveis aleatórias $\boldsymbol{X}_{n}, n=1,2, \ldots$ tais que

$$
\begin{aligned}
\boldsymbol{X}_{n} & =\alpha \log \Delta_{t-1}+\frac{\lambda}{C+V}\left(\boldsymbol{N}_{t}^{(B)}-\boldsymbol{N}_{t}^{(A)}\right) \\
& =\alpha \log \Delta_{t-1}+\frac{\lambda}{n}\left(\boldsymbol{N}_{t}^{(B)}-\boldsymbol{N}_{t}^{(A)}\right)
\end{aligned}
$$

Como $\boldsymbol{X}_{n}=\log \Delta_{t}$, a esperança e a variância de $\boldsymbol{X}_{n}$ são dadas pelo Lema 18:

$$
\begin{aligned}
\mathrm{E}\left[\boldsymbol{X}_{n}\right] & =\mu_{t} \\
& =\alpha \log \Delta_{t-1}+\lambda\left(\frac{1}{1+\nu} \mathfrak{g}_{t-1}-\frac{\nu}{1+\nu} \Psi\left(\frac{b}{\Delta_{t-1}}\right)\right)
\end{aligned}
$$

$$
\begin{aligned}
\operatorname{Var}\left(\boldsymbol{X}_{n}\right) & =\sigma_{n}^{2} \\
& =\frac{\lambda^{2}}{V+C}\left[\frac{1}{1+\nu} \mathfrak{g}_{t-1}\left(1-\mathfrak{g}_{t-1}\right)+\frac{\nu}{1+\nu} \Psi\left(\frac{b}{\Delta_{t-1}}\right)\left(1-\Psi\left(\frac{b}{\Delta_{t-1}}\right)\right)\right] \\
& =\frac{\lambda^{2}}{n}\left[\frac{1}{1+\nu} \mathfrak{g}_{t-1}\left(1-\mathfrak{g}_{t-1}\right)+\frac{\nu}{1+\nu} \Psi\left(\frac{b}{\Delta_{t-1}}\right)\left(1-\Psi\left(\frac{b}{\Delta_{t-1}}\right)\right)\right]
\end{aligned}
$$

Sabemos que $\lambda>0$ é um parâmetro finito. Além disso, sabemos também que $\mathfrak{g}_{t-1} \mathrm{e}$ $\Psi\left(\frac{b}{\Delta_{t-1}}\right)$ são probabilidades e, portanto, estão no intervalo $[0,1]$. Como, por hipótese do teorema, $\nu$ é constante, temos então que

$$
\lim _{n \rightarrow \infty} \operatorname{Var}\left(\boldsymbol{X}_{n}\right)=\lim _{n \rightarrow \infty} \sigma_{n}^{2}=0 .
$$

Usando a desigualdade de Chebyshev ${ }^{2}$, sabemos que,

$$
\forall \varepsilon>0, \quad \mathbb{P}\left(\left|\boldsymbol{X}_{n}-\mu_{t}\right| \geq \varepsilon\right) \leq \frac{\sigma_{n}^{2}}{\varepsilon}
$$

\footnotetext{
${ }^{2}$ Ver, por exemplo, [29]: "If $\boldsymbol{X}$ is a random variable with finite mean $\mu$ and variance $\sigma^{2}$, then for any value $k>0, \mathbb{P}(|\boldsymbol{X}-\mu| \geq k) \leq \frac{\sigma^{2}}{k^{2}} .$,
} 
Como $\lim _{n \rightarrow \infty} \sigma_{n}^{2}=0$, usando o Teorema do confronto ${ }^{3}$, temos que

$$
\lim _{n \rightarrow \infty} \mathbb{P}\left(\left|\boldsymbol{X}_{n}-\mu_{t}\right| \geq \varepsilon\right)=0 \forall \varepsilon>0
$$

ou seja, $\boldsymbol{X}_{n} \stackrel{\mathbb{P}}{\rightarrow} \mu_{t}$.

Assim, quando o número de agentes aumenta, a variável $\log \Delta_{t} \mid \boldsymbol{P}_{t-1}, \Delta_{t-1}$ converge para uma variável aleatória degenerada no valor da sua esperança, i.e., converge para um valor determinístico.

\subsubsection{Preço de mercado}

Lembramos que o preço de mercado no instante $t$ é dado por $\boldsymbol{P}_{t}=\frac{1}{\boldsymbol{n}_{t}} \sum_{k=1}^{\boldsymbol{n}_{t}} \boldsymbol{T}_{t}^{(k)}$. Já conhecemos a distribuição de $\boldsymbol{T}_{t}^{(k)} \mid \boldsymbol{P}_{t-1}, \Delta_{t-1}$, que é dada por (2.5.4), e sabemos, pelo Corolário 16, que a sua esperança e a sua variância são finitas. Sabemos também que $\boldsymbol{n}_{t}=\min \left\{\boldsymbol{N}_{t}^{(A)}, \boldsymbol{N}_{t}^{(B)}\right\}$ é aleatório, e sua distribuição não pode ser escrita de forma explícita.

Observe que o preço de mercado num dado instante é simplesmente a média das transações ocorridas naquele instante. Aparentemente, seria possível aplicar o Teorema Central do Limite para obter a distribuição assintótica de $\boldsymbol{P}_{t} \mid \boldsymbol{P}_{t-1}, \Delta_{t-1}$. Porém, como o número de elementos da soma que compõe $\boldsymbol{P}_{t}$ também é aleatório, não é possível usar diretamente o Teorema Central do Limite para obter a distribuição assintótica de $\boldsymbol{P}_{t} \mid \boldsymbol{P}_{t-1}, \Delta_{t-1}$. Também por este mesmo motivo, não podemos usar a Lei Forte dos Grandes Números neste caso.

Mas, como veremos nesta seção, nem tudo está perdido: é possível obter a convergência em probabilidade de $\boldsymbol{P}_{t} \mid \boldsymbol{P}_{t-1}, \Delta_{t-1}$ para a sua esperança condicional, num resultado similar à Lei Forte, mesmo com $\boldsymbol{n}_{t}$ aleatório. E, para fazer isso, vamos precisar dos resultados apresentados a seguir, começando pela esperança e pela variância de $\boldsymbol{P}_{t} \mid \boldsymbol{P}_{t-1}, \Delta_{t-1}$. Mas, antes disso, introduziremos a seguinte notação:

$$
\begin{aligned}
\mathrm{E}_{t-1}[\cdot] & :=\mathrm{E}\left[\cdot \mid \boldsymbol{P}_{t-1}, \Delta_{t-1}\right], \\
\operatorname{Var}_{t-1}(\cdot) & :=\operatorname{Var}\left(\cdot \mid \boldsymbol{P}_{t-1}, \Delta_{t-1}\right) .
\end{aligned}
$$

Lema 20. $\mathrm{E}_{t-1}\left[\boldsymbol{P}_{t}\right]=\mathrm{E}_{t-1}\left[\boldsymbol{T}_{t}^{(k)}\right]$.

\footnotetext{
${ }^{3}$ Teorema 4, página 198 de [30]: "Sejam $\mathcal{X} \in \mathbb{R}, f, g, h: \mathcal{X} \rightarrow \mathbb{R}, a \in \mathcal{X}$. Se, para todo $x \in \mathcal{X}, x \neq a$, for $f(x) \leq g(x) \leq h(x)$ e, além disso, tivermos $\lim _{x \rightarrow a} f(x)=\lim _{x \rightarrow a} h(x)=L$, então $\lim _{x \rightarrow a} g(x)=L$."
} 
Demonstração. Ver apêndice B.4.

Como a distribuição de $\boldsymbol{T}_{t}^{(k)} \mid \boldsymbol{P}_{t-1}, \Delta_{t-1}$ é conhecida e dada por (2.5.4), podemos agora usar o Lema 20 para calcular o valor da esperança (condicionada ao passado) do preço de mercado:

Teorema 21. A esperança do preço de mercado é dada por

$$
\mathrm{E}_{t-1}\left[\boldsymbol{P}_{t}\right]=\mathfrak{h}_{\Psi, b}\left(\Delta_{t-1}\right) \boldsymbol{P}_{t-1},
$$

$\operatorname{com} \mathfrak{h}_{\Psi, b}\left(\Delta_{t}\right)=b-\frac{1}{\Psi\left(\frac{b}{\Delta_{t}}\right)} \int_{0}^{b} \Psi\left(\frac{\xi}{\Delta_{t}}\right) d \xi$

Demonstração. Ver apêndice B.5.

A função

$$
\mathfrak{h}_{\Psi, b}\left(\Delta_{t}\right):=b-\frac{1}{\Psi\left(\frac{b}{\Delta_{t}}\right)} \int_{0}^{b} \Psi\left(\frac{\xi}{\Delta_{t}}\right) d \xi,
$$

que relaciona a esperança do preço no instante atual com o preço no instante anterior, será muito importante na análise da dinâmica do sistema. Este ponto será discutido na Seção 3.3. Mas, por enquanto, ela é apenas a função que dá a esperança de $\boldsymbol{P}_{t} \mid \boldsymbol{P}_{t-1}, \Delta_{t-1}$.

Para chegar ao resultado de convergência desta seção, porém, precisaremos, ainda, conhecer a variância de $\boldsymbol{P}_{t} \mid \boldsymbol{P}_{t-1}, \Delta_{t-1}$ :

Lema 22. $\operatorname{Var}_{t-1}\left(\boldsymbol{P}_{t}\right)=\operatorname{Var}_{t-1}\left(\boldsymbol{T}_{t}^{(k)}\right) \mathrm{E}_{t-1}\left[\frac{1}{\boldsymbol{n}_{t}}\right]$.

Demonstração. Ver apêndice B.6.

Um outro lema será necessário para obter a convergência de $\boldsymbol{P}_{t} \mid \boldsymbol{P}_{t-1}, \Delta_{t-1}$ quando o número de agentes aumenta:

Lema 23. Seja $n=V+C$ o número total de agentes e considere que a razão $V / C=\nu$ é constante. Então

$$
\lim _{n \rightarrow \infty} \mathrm{E}_{t-1}\left[\frac{1}{\boldsymbol{n}_{t}}\right]=0
$$

Demonstração. Ver apêndice B.7.

Assim como foi feito para $\Delta_{t} \mid \boldsymbol{P}_{t-1}, \Delta_{t-1}$ no Teorema 19, vamos agora obter uma propriedade assintótica de $\boldsymbol{P}_{t} \mid \boldsymbol{P}_{t-1}, \Delta_{t-1}$, que nos permitirá analisar a dinâmica do sistema em questão. Para isso, vamos usar os Lemas 22 e 23. 
Teorema 24. Seja $n=V+C$ o número total de agentes e considere que a razão $V / C=\nu$ é constante. Então

$$
\boldsymbol{P}_{t} \mid \boldsymbol{P}_{t-1}, \Delta_{t-1} \underset{n \rightarrow \infty}{\stackrel{\mathbb{P}}{\longrightarrow}} \mathfrak{h}_{\Psi, b}\left(\Delta_{t-1}\right) \boldsymbol{P}_{t-1}
$$

Demonstração. Primeiramente, lembramos do resultado do Teorema 21, que nos dá a esperança de $\boldsymbol{P}_{t} \mid \boldsymbol{P}_{t-1}, \Delta_{t-1}$, aqui denotada por $\eta_{t}$ :

$$
\eta_{t}:=\mathrm{E}_{t-1}\left[\boldsymbol{P}_{t}\right]=\mathfrak{h}_{\Psi, b}\left(\Delta_{t-1}\right) \boldsymbol{P}_{t-1}
$$

Agora, pelo Lema 22, a variância de $\boldsymbol{P}_{t} \mid \boldsymbol{P}_{t-1}, \Delta_{t-1}$, aqui denotada por $\varsigma_{t}^{2}$, é dada por

$$
\varsigma_{t}^{2}:=\operatorname{Var}_{t-1}\left(\boldsymbol{P}_{t}\right)=\operatorname{Var}_{t-1}\left(\boldsymbol{T}_{t}^{(k)}\right) \mathrm{E}_{t-1}\left[\frac{1}{\boldsymbol{n}_{t}}\right]
$$

Usando o Lema 23, e lembrando que, pelo Corolário 16, $\operatorname{Var}_{t-1}\left(\boldsymbol{T}_{t}^{(k)}\right)<\infty$, temos que

$$
\begin{aligned}
\lim _{n \rightarrow \infty} \varsigma_{t}^{2} & =\operatorname{Var}_{t-1}\left(\boldsymbol{T}_{t}^{(k)}\right) \lim _{n \rightarrow \infty} \mathrm{E}_{t-1}\left(\frac{1}{\boldsymbol{n}_{t}}\right) \\
& =0 .
\end{aligned}
$$

Pela desigualdade de Chebyshev, sabemos que,

$$
\forall \varepsilon>0, \quad \mathbb{P}_{t-1}\left(\left|\boldsymbol{P}_{t}-\eta_{t}\right| \geq \varepsilon\right) \leq \frac{\varsigma_{t}^{2}}{\varepsilon}
$$

Como $\lim _{n \rightarrow \infty} \varsigma_{n}^{2}=0$, usando o Teorema do confronto, temos que

$$
\lim _{n \rightarrow \infty} \mathbb{P}_{t-1}\left(\left|\boldsymbol{P}_{t}-\eta_{t}\right| \geq \varepsilon\right)=0 \forall \varepsilon>0
$$

ou seja, $\boldsymbol{P}_{t} \underset{n \rightarrow \infty}{\stackrel{\mathbb{P}}{\longrightarrow}} \eta_{t}$.

\subsection{Dinâmica dos preços}

Nesta seção, vamos analisar a dinâmica dos preços usando os resultados obtidos nos Teoremas 19 e 24 , sobre a convergência de $\Delta_{t}$ e de $\boldsymbol{P}_{t}$ quando o número de agentes é grande. Este resultado de convergência é similar ao obtido por Prado [31] Dawid [32]. Essa convergência é o que nos permitirá analisar a dinâmica do processo $\boldsymbol{P}_{t}$ a partir do sistema dinâmico determinístico a que o processo converge. 
Usando então os Teoremas 19 e 24, para um número grande de agentes, o processo é aproximado pelo seguinte sistema dinâmico:

$$
\left\{\begin{array}{l}
p_{t}=p_{t-1} \mathfrak{h}_{\Psi, b}\left(\delta_{t-1}\right) \\
\log \delta_{t}=\alpha \log \delta_{t-1}+\lambda\left(\frac{1}{1+\nu} \mathfrak{g}\left(p_{t-1} ; b, k, R\right)-\frac{\nu}{1+\nu} \Psi\left(\frac{b}{\delta_{t-1}}\right)\right)
\end{array}\right.
$$

com $p_{t}$ e $\delta_{t}$ os valores determinísticos para os quais os processos $\boldsymbol{P}_{t}$ e $\Delta_{t}$ convergem quando o número de agentes aumenta.

Esse sistema de equações a diferenças é homogêneo de segunda ordem, mas é não-linear. Portanto, a sua solução não pode ser obtida de forma exata por meio das técnicas já conhecidas aplicáveis a sistemas lineares. Porém, vamos estudar o seu comportamento nos valendo de suas características específicas.

A primeira equação nos dá a tendência dos preços a cada instante em função do estado anterior, e a segunda nos dá o comportamento da demanda. Na seção 3.3.1, vamos analisar como os preços se comportam a depender da forma de $\mathfrak{h}_{\Psi, b}(\cdot)$. Em seguida, na seção 3.3.2, vamos obter as condições sob as quais existe um preço de equilíbrio e qual é o seu valor.

\subsubsection{Tendência do preço de mercado}

O preço de mercado é descrito pela seguinte equação a diferenças:

$$
p_{t}=\mathfrak{h}_{\Psi, b}\left(\delta_{t-1}\right) p_{t-1},
$$

$\operatorname{com} \mathfrak{h}_{\Psi, b}(\delta)=b-\frac{1}{\Psi\left(\frac{b}{\Delta_{t}}\right)} \int_{0}^{b} \Psi\left(\frac{\xi}{\Delta_{t}}\right) d \xi($ ver Teoremas 21 e 24$)$

Observe que, quando $\mathfrak{h}_{\Psi, b}\left(\delta_{t-1}\right)>1$, o preço no instante $t$ será maior que o preço em $t-1$. Da mesma forma, se $\mathfrak{h}_{\Psi, b}\left(\delta_{t-1}\right)=1$, o preço não se altera e, se $\mathfrak{h}_{\Psi, b}\left(\delta_{t-1}\right)<1$, o preço irá diminuir.

Para ilustrar como uma mudança em $\delta_{t}$ pode modificar a tendência dos preços, note que a condição de aumento do preço, $\mathfrak{h}_{\Psi, b}\left(\delta_{t-1}\right)>1$, pode ser reescrita da seguinte maneira:

$$
(b-1) \Psi\left(\frac{b}{\delta_{t-1}}\right)>\int_{0}^{1} \Psi\left(\frac{\xi}{\delta_{t-1}}\right) d \xi+\int_{1}^{b} \Psi\left(\frac{\xi}{\delta_{t-1}}\right) d \xi
$$

Esta situação é ilustrada na Figura 3.3.1. Quando a área da região I é maior que a área da região II, o preço aumenta. Quando as duas áreas são iguais, o preço fica estável e, se a área da região I for menor que a da região II, o preço irá diminuir. As mudanças na demanda 


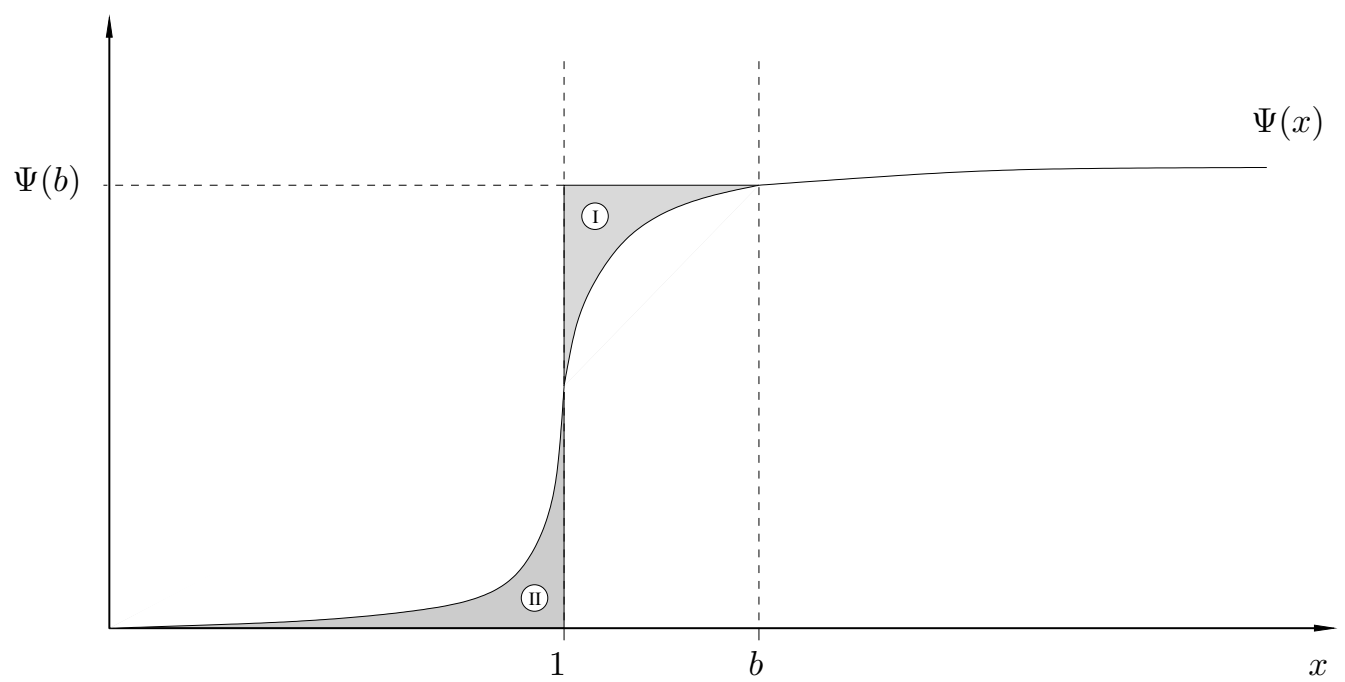

(a) $\Delta_{t-1}=1$ e estabilidade dos preços.

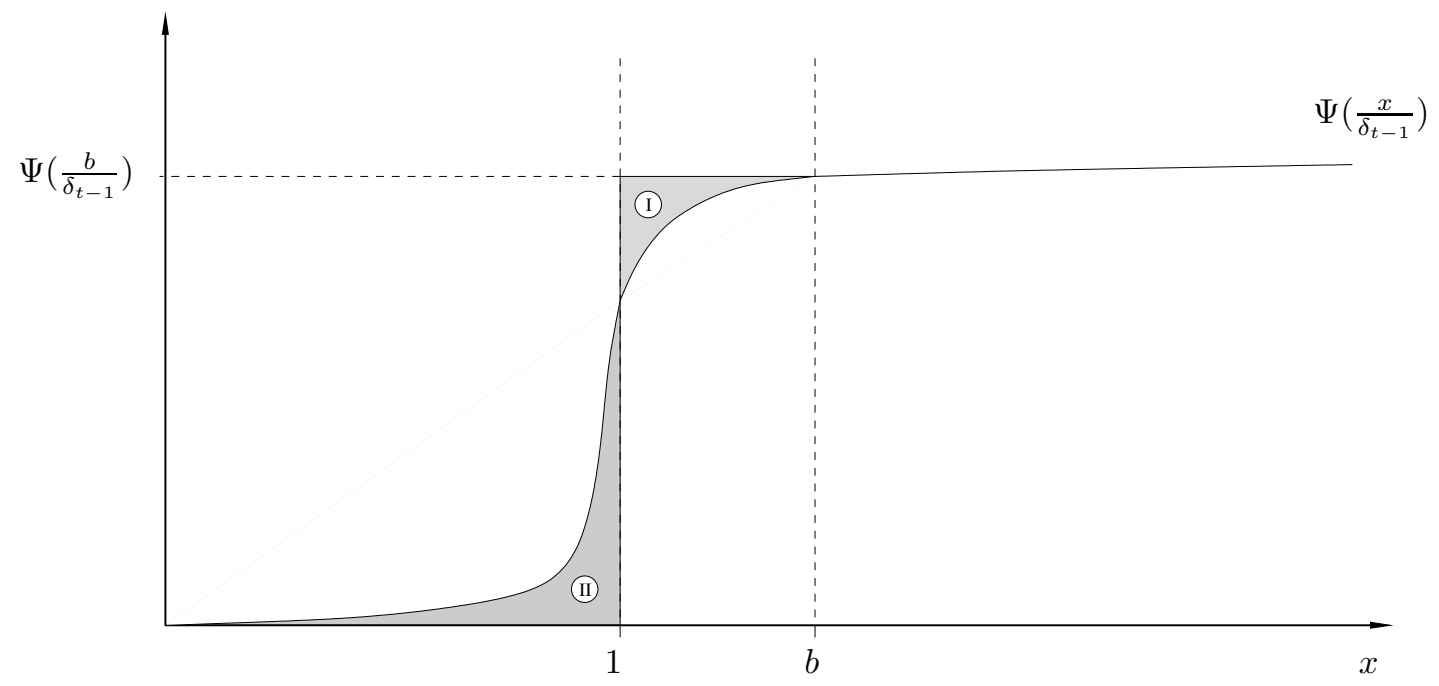

(b) $\Delta_{t-1}<1$ faz a tendência dos preços mudar para redução.

Figura 3.3.1: Condição para haver tendência de aumento do preço.

podem inverter essa tendência. Por exemplo, se $\delta_{t-1}<1$, então uma tendência de aumento do preço pode ser revertida para uma tendência de redução. Por outro lado, um aumento na demanda pode levar a uma tendência de aumento do preço, ou intensificá-la caso a tendência já fosse de aumento.

Vamos agora estudar o comportamento de $\mathfrak{h}_{\Psi, b}(\delta)$ para responder à seguinte questão: existe um valor de $\delta^{\text {crítico }}$ para o qual o preço de mercado é estável? Ou, em outras palavras, existe $\delta^{\text {crítico }}$ tal que $\mathfrak{h}_{\Psi, b}\left(\delta^{\text {crítico }}\right)=1$ ?

A resposta a essa questão depende do comportamento dos compradores (representado pelo parâmetro $b$ ), e também das características dos vendedores (representados pela distribuição $\Psi)$. Nas proposições a seguir, mostraremos que, dependendo do comportamento dos compradores, o preço de mercado sempre irá diminuir, independentemente dos valores que $\delta_{t}$ assuma. Sob certas condições, porém, verificaremos que o preço no instante $t$ pode aumen- 


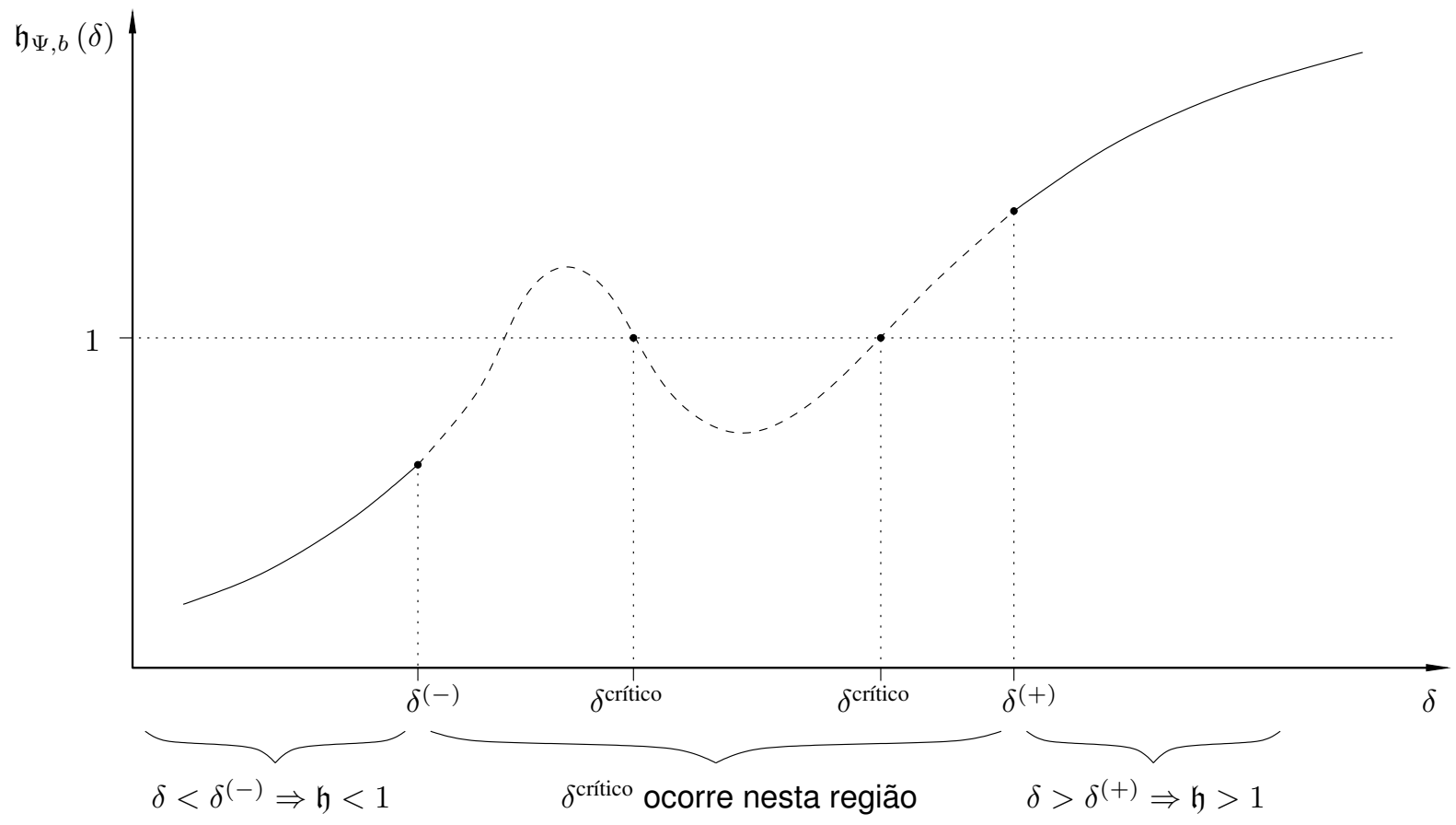

Figura 3.3.2: A função $\mathfrak{h}_{\Psi, b}(\delta)$, quando $b>1$ e $\lim _{x \rightarrow 0} \frac{x \psi(x)}{\Psi(x)}=c>\frac{1}{b-1}$.

tar ou diminuir, dependendo do valor de $\delta_{t-1}$.

A Figura 3.3.2 ilustra os resultados que serão obtidos nas Proposições 26 e 27, e também o resultado do Corolário 28, que garante a existência de um $\delta^{\text {crítico }} \in\left[\delta^{(-)}, \delta^{(+)}\right]$sob o qual o preço fica estável. As proposições mostram que, na direção de $\delta$ pequeno (demanda fraca), o preço irá diminuir, enquanto que na direção de $\delta$ grande (demanda forte), o preço irá aumentar. Entre as duas situações, existirá (pelo menos) um valor de $\delta$ para o qual a função $\mathfrak{h}_{\Psi, b}(\delta)$ cruza o valor 1 , ou seja, existe um $\delta^{\text {crítico }}$ que estabiliza o preço.

Proposição 25. Compradores excessivamente pessimistas $(b \leq 1)$.

Quando $b \leq 1, \mathfrak{h}_{\Psi, b}(\delta)<1$ qualquer que seja o valor de $\delta>0$, ou seja, não existe nenhum valor de demanda que reverta a tendência de queda do preço.

Demonstração. De (3.2.5), a condição para que o preço diminua, $\mathfrak{h}_{\Psi, b}(\delta)<1$, pode ser reescrita como

$$
\frac{\int_{0}^{b} \Psi\left(\frac{\xi}{\delta}\right) d \xi}{\Psi\left(\frac{b}{\delta}\right)}>b-1 .
$$

Pela Hipótese 7, $\Psi(x)>0 \forall x>0$. Assim, o primeiro lado dessa desigualdade está definido e é sempre maior que zero quando $b>0$, ou seja,

$$
\frac{\int_{0}^{b} \Psi\left(\frac{\xi}{\delta}\right) d \xi}{\Psi\left(\frac{b}{\delta}\right)}>0, \forall \Delta>0, b>0 .
$$

Como $b \leq 1 \Leftrightarrow b-1 \leq 0$, a desigualdade (3.3.3) é sempre satisfeita para $b \leq 1$. 
Quando os compradores são pessimistas ( $b<1$ ), a Proposição 25 nos mostra que, fixados os parâmetros do sistema e dado um preço inicial maior que zero, o preço de mercado irá apenas diminuir com o decorrer do tempo, até chegar a zero. Obviamente, essa dinâmica não é verificada na realidade. Precisamos verificar outras situações não triviais, que serão apresentadas nas proposições 26 e 27.

Antes de apresentá-las, precisaremos de uma nova hipótese, a saber:

Hipótese 8. A variável aleatória $\boldsymbol{a}^{(i)}$ é contínua.

Vamos denotar a função densidade de $\boldsymbol{a}^{(i)}$ por $\psi(x)$.

Proposição 26. Demanda fraca resulta em redução do preço.

Sob a Hipótese 8, existe um valor de demanda $\delta^{(-)}$tal que $\mathfrak{h}_{\Psi, b}(\delta)<1$ para todo $\delta<\delta^{(-)}$, ou seja, existe uma demanda suficientemente fraca que resulta em redução do preço de mercado.

Demonstração. Ver apêndice B.8.

Proposição 27. Demanda forte resulta em aumento do preço.

Sob a Hipótese 8 , se $b>1$ e se $\Psi$ for tal que $\lim _{x \rightarrow 0} \frac{x \psi(x)}{\Psi(x)}=c>\frac{1}{b-1}$, então existe um valor de demanda $\delta^{(+)}$tal que $\mathfrak{h}_{\Psi, b}(\delta)>1$ para todo $\delta>\delta^{(+)}$, ou seja, existe uma demanda suficientemente forte que resultará em aumento do preço de mercado.

Demonstração. Ver apêndice B.9.

A Proposição 26 nos mostra que, mesmo quando $b>1$, existe algum valor de demanda suficientemente fraca capaz de reverter o aumento do preço. Já a Proposição 27 nos garante que, se $\Psi$ for uma distribuição tal que $\lim _{x \rightarrow 0} \frac{x \psi(x)}{\Psi(x)}=c>\frac{1}{b-1}$, existe algum valor de demanda suficientemente forte capaz de reverter a queda do preço. Quando essas duas situações - de redução ou de aumento do preço - são possíveis, a dinâmica do preço dependerá da dinâmica da própria demanda.

Corolário 28. Sob a Hipótese 8 , se $b>1$ e se $\lim _{x \rightarrow 0} \frac{x \psi(x)}{\Psi(x)}=c>\frac{1}{b-1}$, então existe um valor de demanda $\delta^{\text {crítico }}$ no intervalo $\left[\delta^{(-)}, \delta^{(+)}\right]$tal que $\mathfrak{h}_{\Psi, b}\left(\delta^{\text {crítico }}\right)=1$, ou seja, existe $\delta^{\text {crítico }}>0$ tal que o preço de mercado fica estável em relação ao preço no instante anterior.

Demonstração. Decorre trivialmente das proposições 26 e 27 e da aplicação do Teorema do Valor Intermediário 4 à função $\mathfrak{h}_{\Psi, b}(\delta)$.

\footnotetext{
${ }^{4}$ Teorema do Valor Intermediário [30]: "Seja $f:[a, b] \rightarrow \mathbb{R}$ contínua. Se $f(a)<d<f(b)$ então existe $c \in(a, b)$ tal que $f(c)=d . "$
} 
O exemplo a seguir ilustra uma aplicação da Proposição 27 e do Corolário 28 a uma distribuição $\Psi$ específica.

Exemplo 29. Seja $\boldsymbol{X}$ uma variável aleatória com distribuição de Dagum de parâmetros $\alpha>$ $0, \beta>0$ e $\gamma>0$. O suporte dessa variável é $\mathbb{R}_{+}$e sua função de distribuição acumulada e função densidade são dadas por [33]

$$
\begin{aligned}
& \Psi(x)=\left[1+\left(\frac{x}{\beta}\right)^{-\alpha}\right]^{-\gamma} \\
& \psi(x)=\frac{\alpha \gamma\left(\frac{x}{\beta}\right)^{\alpha \gamma}}{x\left[\left(\frac{x}{\beta}\right)^{\alpha}+1\right]^{\gamma+1}} .
\end{aligned}
$$

Note que

$$
\begin{aligned}
\lim _{x \rightarrow 0} \frac{x \psi(x)}{\Psi(x)} & =\lim _{x \rightarrow 0} x \frac{\alpha \gamma\left(\frac{x}{\beta}\right)^{\alpha \gamma}}{x\left[\left(\frac{x}{\beta}\right)^{\alpha}+1\right]^{\gamma+1}}\left[1+\left(\frac{x}{\beta}\right)^{-\alpha}\right]^{\gamma} \\
& =\lim _{x \rightarrow 0} \alpha \gamma \frac{x^{\alpha \gamma} \beta^{-\alpha \gamma}}{\left(x^{\alpha} \beta^{-\alpha}+1\right)^{\gamma+1}}\left(1+x^{-\alpha} \beta^{\alpha}\right)^{\gamma} \\
& =\lim _{x \rightarrow 0} \alpha \gamma \frac{\beta^{-\alpha \gamma}}{\left(x^{\alpha} \beta^{-\alpha}+1\right)^{\gamma+1}} x^{\alpha \gamma}\left[x^{-\alpha}\left(x^{\alpha}+\beta^{\alpha}\right)\right]^{\gamma} \\
& =\alpha \gamma .
\end{aligned}
$$

Se $\boldsymbol{a}^{(i)} \sim \operatorname{Dagum}(\alpha, \beta, \gamma)$, usando o Corolário 28, podemos garantir que existirá um $\delta^{\text {crítico }}>$ 0 tal que $\mathfrak{h}_{\Psi, b}\left(\delta^{\text {crítico }}\right)=1$ quando os parâmetros $\alpha$ e $\gamma$ satisfazem a desigualdade

$$
\alpha \gamma>\frac{1}{b-1} \Leftrightarrow b>\frac{1}{\alpha \gamma}+1
$$

Lembramos que o parâmetro $b$ representa o quanto os compradores estão dispostos a pagar a mais pelos imóveis do que o preço de mercado do instante anterior. Assim, para valores de $b$ próximos de 1 , notamos que o produto $\alpha \gamma$ deverá ser grande para que a desigualdade seja satisfeita, garantindo a existência de uma demanda que produza aumento e estabilidade do preço de mercado.

Mais um exemplo, agora numérico, ilustra o comportamento da função $\mathfrak{h}_{\Psi, b}(\delta)$.

Exemplo 30. Considere novamente a função de distribuição de Dagum, dada por (3.3.4), 
com os seguintes valores para os seus parâmetros:

$$
\begin{aligned}
& \alpha=14,549 \\
& \beta=0,9 \\
& \gamma=5,0186
\end{aligned}
$$

A Figura 3.3.3 mostra o comportamento da função $\mathfrak{h}_{\Psi, b}(\delta)$ para diversos valores do parâmetro $b$. Lembre-se de que o parâmetro $b$ é o que define o preço de reserva dos compradores. Sobre este gráfico, observe que

1. quando $b=1$, temos a curva vermelha, que representa o caso da Proposição 25, quando os compradores são excessivamente pessimistas. Nesta situação, note que $\mathfrak{h}_{\Psi, b}(\delta)$ fica abaixo de 1 para todo $\delta$, ou seja, não há um valor de demanda que produza estabilidade dos preços;

2. quando $b=1+\frac{1}{\alpha \gamma}$, temos a curva azul escura, que se aproxima de 1 quando $\delta$ aumenta. Esta situação ilustra a situação descrita na Proposição 27, em que vimos que $\lim _{x \rightarrow 0} \frac{x \psi(x)}{\Psi(x)}=\alpha \gamma>\frac{1}{b-1}$ é condição suficiente para que $\mathfrak{h}_{\Psi, b}(\delta)>1$ para algum valor valor de $\delta$. Quando $b=1+\frac{1}{\alpha \gamma}$, a condição não é atendida, e observamos que $\mathfrak{h}_{\Psi, b}(\delta)$ se aproxima de 1 mas não cruza este limiar ${ }^{5}$;

3. quando $b>1+\frac{1}{\alpha \gamma}$, temos as curvas verde e azul clara. Nestes dois casos, a condição suficiente estabelecida na Proposição 27 é atendida e, como era esperado, observamos que $\mathfrak{h}_{\Psi, b}(\delta)$ cruza o 1

4. o valor de $\delta^{\text {crítico }}$ (aquele em que a função $\mathfrak{h}_{\Psi, b}(\delta)=1$ ) é diferente para cada valor de $b$ escolhido, ou seja, ele depende do parâmetro $b$. E ainda, dependendo do valor de $b$, o $\delta^{\text {crítico }}$ pode ser maior, menor ou igual a 1 , sendo este último caso ilustrado pela curva verde;

5. para valores de $\delta$ grandes, a função $\mathfrak{h}_{\Psi, b}(\delta)$ se aproxima de $b$ : o valor exato desse limite é dado por (B.9.1), ou seja, $\lim _{\delta \rightarrow \infty} \mathfrak{h}_{\Psi, b}(\delta)=b \frac{c}{1+c}, c=\lim _{x \rightarrow 0} \frac{x \psi(x)}{\Psi(x)}$. No caso deste exemplo, $c=\alpha \gamma$, o que nos dá $\lim _{\delta \rightarrow \infty} \mathfrak{h}_{\Psi, b}(\delta) \approx b$.

No Exemplo 30, observamos que $\delta^{\text {crítico }}$ é único, e seu valor diminui quando $b$ aumenta. Porém, observe que o Corolário 28 nos garante a existência de um $\delta^{\text {crítico }}$ tal que $\mathfrak{h}_{\Psi, b}\left(\delta^{\text {crítico }}\right)=$ 1, mas não nos garante que este valor é único em geral. A proposição a seguir nos dá a condição para que $\delta^{\text {crítico }}$ seja único:

\footnotetext{
${ }^{5} \mathrm{Na}$ Proposição 27, mostramos que $\lim _{x \rightarrow 0} \frac{x \psi(x)}{\Psi(x)}=\alpha \gamma>\frac{1}{b-1}$ é condição suficiente para que o limiar de 1 seja ultrapassado, mas não que é condição necessária. Porém, neste caso específico, observamos que, nas situações em que a condição não é atendida, o limiar realmente não foi ultrapassado.
} 


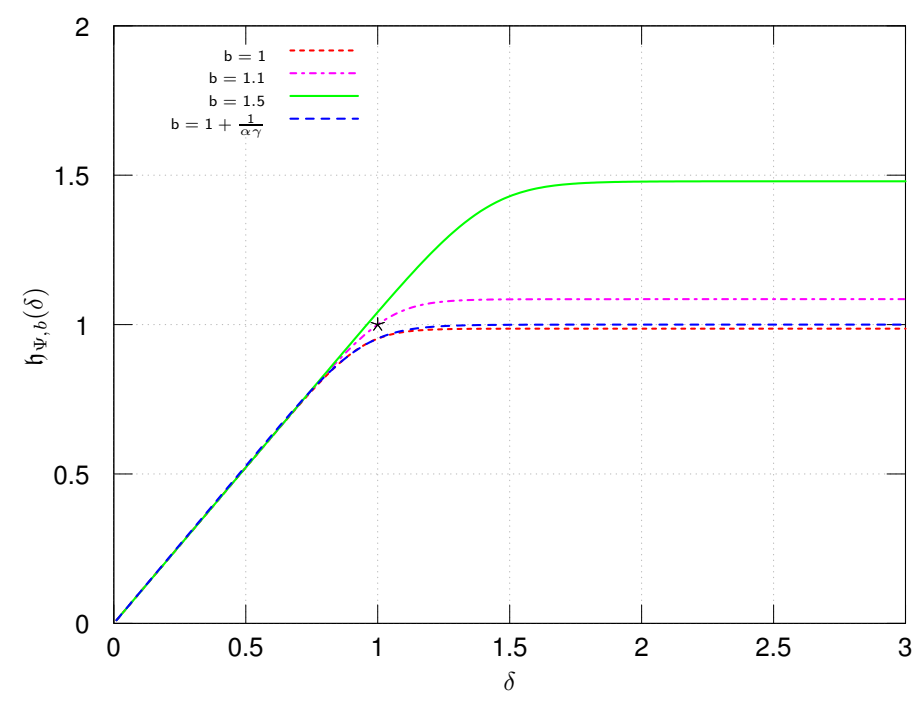

Figura 3.3.3: Gráfico da função $\mathfrak{h}_{\Psi, b}(\delta)$ para $\Psi(x)=\left[1+\left(\frac{x}{\beta}\right)^{-\alpha}\right]^{-\gamma}$ e vários valores de $b$.

Proposição 31. Sob a Hipótese 8, se b > 1 e se $\Psi$ for tal que

1. $\lim _{x \rightarrow 0} \frac{x \psi(x)}{\Psi(x)}=c>\frac{1}{b-1}$

2. $\int_{0}^{x} \Psi(\xi) d \xi<\frac{x \Psi(x)}{1+\frac{x \psi(x)}{\Psi(x)}}$,

então o valor $\delta^{\text {crítico }}$ tal que $\mathfrak{h}_{\Psi, b}\left(\delta^{\text {crítico }}\right)=1$ existe e é único.

Demonstração. Ver apêndice B.10.

\subsubsection{Preço de equilíbrio}

Lembramos que o sistema dinâmico para o qual converge o processo é descrito pelas seguintes equações a diferenças:

$$
\begin{gathered}
p_{t}=p_{t-1} \mathfrak{h}_{\Psi, b}\left(\delta_{t-1}\right) \\
\log \delta_{t}=\alpha \log \delta_{t-1}+\lambda\left(\frac{1}{1+\nu} \mathfrak{g}\left(p_{t-1} ; b, k, R\right)-\frac{\nu}{1+\nu} \Psi\left(\frac{b}{\delta_{t-1}}\right)\right)
\end{gathered}
$$

Se o sistema dinâmico descrito por (3.3.6) e (3.3.7) tiver um ponto de equilíbrio (assintoticamente estável), $\left(p^{\star}, \delta^{\star}\right)$, ele será tal que [34]

$$
p_{t}-p_{t-1}=0
$$




$$
\delta_{t}-\delta_{t-1}=0
$$

quando $t \rightarrow \infty$.

Da equação (3.3.6), temos que

$$
p_{t}-p_{t-1}=0 \Leftrightarrow \mathfrak{h}_{\Psi, b}\left(\delta_{t-1}\right)=1 \text { ou } p_{t}=p_{t-1}=0 .
$$

A solução trivial $p^{\star}=0$ não nos interessa. Assim, $\delta^{\star}$ deve ser tal que

$$
\mathfrak{h}_{\Psi, b}\left(\delta^{\star}\right)=1 .
$$

Na seção 3.3.1, vimos que, quando $b>0$ e $\lim _{x \rightarrow 0} \frac{x \psi(x)}{\Psi(x)}=c>\frac{1}{b-1}$, o Corolário 28 nos garante que existe um valor de demanda que pode produzir a estabilidade no preço de mercado. Ou seja, ele garante que existe um $\delta^{\star}=\delta^{\text {crítico. }}$. E ainda, se $\int_{0}^{x} \Psi(\xi) d \xi<\frac{x \Psi(x)}{1+\frac{x \psi(x)}{\Psi(x)}}$, a Proposi-

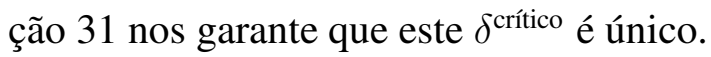

Garantida a existência de $\delta^{\star}=\delta^{\text {crítico }}$, a existência do preço de equilíbrio (diferente de zero) depende também da equação (3.3.7), o que nos leva à seguinte proposição:

Proposição 32. Se $\frac{(1+\nu)(1-\alpha)}{\lambda} \log \delta^{\text {crítico }}+\nu \Psi\left(\frac{b}{\delta \text { critico }}\right)>1$, então não existe preço de equilíbrio além da solução trivial $p^{\star}=0$.

Demonstração. A equação (3.3.7) nos dá, de forma implícita, o valor de $p^{\star}$ :

$$
\begin{aligned}
& \log \delta^{\star}=\alpha \log \delta^{\star}+\lambda\left(\frac{1}{1+\nu} \mathfrak{g}\left(p^{\star} ; b, k, R\right)-\frac{\nu}{1+\nu} \Psi\left(\frac{b}{\delta^{\star}}\right)\right) \\
\Leftrightarrow & (1-\alpha) \log \delta^{\star}=\frac{\lambda}{1+\nu}\left(\mathfrak{g}\left(p^{\star} ; b, k, R\right)-\nu \Psi\left(\frac{b}{\delta^{\star}}\right)\right) \\
\Leftrightarrow & \mathfrak{g}\left(p^{\star} ; b, k, R\right)=\frac{(1+\nu)(1-\alpha)}{\lambda} \log \delta^{\star}+\nu \Psi\left(\frac{b}{\delta^{\star}}\right)
\end{aligned}
$$

Note que a função $\mathfrak{g}(p ; b, k, R)$ é uma probabilidade. Portanto, a sua imagem é o conjunto $[0,1]$. Por isso, se os valores dos parâmetros $\nu, \alpha$ e $\lambda$ forem tais que

$$
\frac{(1+\nu)(1-\alpha)}{\lambda} \log \delta^{\star}+\nu \Psi\left(\frac{b}{\delta^{\star}}\right)>1
$$

não haverá nenhum valor de $p$ que seja solução de (3.3.9). 
Deste resultado cabe ainda uma interpretação muito importante: quando os parâmetros são tais que vale a desigualdade

$$
\frac{(1+\nu)(1-\alpha)}{\lambda} \log \delta^{\star}+\nu \Psi\left(\frac{b}{\delta^{\star}}\right)>1,
$$

então não existirá preço de equilíbrio baixo o suficiente para resolver a equação (3.3.9), pois o preço mais baixo possível, que é zero, produz $\mathfrak{g}_{t-1}=1 \mathrm{e}$, portanto, não é solução para a equação.

A Proposição 31 nos garante a existência de um $\delta^{\star}=\delta^{\text {crítico }}$ único. Já a Proposição 32 nos garante a existência de um $p^{\star}$ de equilíbrio dado $\delta^{\star}=\delta^{\text {crítico }}$. Mas nos resta, ainda, verificar se este ponto equilíbrio não trivial é único. Para isso, precisaremos da seguinte hipótese:

Hipótese 9. Na região $\mathbb{R}_{+} \times \mathbb{R}_{+}$, a densidade conjunta de renda e riqueza $f_{Y, W}(y, w)$ é não nula em quase toda parte.

Com esta hipótese, temos o seguinte resultado sobre a existência e unicidade do ponto de equilíbrio não trivial:

Proposição 33. Se $\Psi$ for tal que

$$
\begin{aligned}
& \text { 1. } \lim _{x \rightarrow 0} \frac{x \psi(x)}{\Psi(x)}=c>\frac{1}{b-1} \\
& \text { 2. } \int_{0}^{x} \Psi(\xi) d \xi<\frac{x \Psi(x)}{1+\frac{x \psi(x)}{\Psi(x)}}
\end{aligned}
$$

e os parâmetros $\nu, \alpha, \lambda$ e b forem tais que

$$
\frac{(1+\nu)(1-\alpha)}{\lambda} \log \delta^{\text {crítico }}+\nu \Psi\left(\frac{b}{\delta^{\text {crítico }}}\right) \leq 1
$$

com $\delta^{\text {crítico }}$ tal que $\mathfrak{h}_{\Psi, b}\left(\delta^{\text {crítico }}\right)=1$, e ainda, se a densidade de renda e riqueza atender a Hipótese 9, então o ponto de equilíbrio não trivial $\left(p^{\star}, \delta^{\star}\right)$ existe e é único. Além disso, ele será dado por

$$
\begin{aligned}
\delta^{\star} & =\delta^{\text {crítico }}, \mathfrak{h}_{\Psi, b}\left(\delta^{\text {crítico }}\right)=1, \\
p^{\star} & =\mathfrak{g}^{-1}\left(\frac{(1+\nu)(1-\alpha)}{\lambda} \log \delta^{\star}+\nu \Psi\left(\frac{b}{\delta^{\star}}\right)\right),
\end{aligned}
$$

com $\mathfrak{g}^{-1}(\cdot)$ a inversa de $\mathfrak{g}(p ; b, k, R)$ em $p$.

Demonstração. A Proposição 31 nos garante que, se $\Psi$ atender às condições (1) e (2), então o valor de $\delta$ no ponto de equilíbrio será único e dado por $\delta^{\star}=\delta^{\text {crítico. }}$. 
Além disso, sabemos que $p$ no ponto de equilíbrio é dado implicitamente por (3.3.7) para $\delta_{t}=\delta_{t-1}=\delta^{\star}$, ou seja,

$$
\begin{aligned}
\log \delta^{\star}=\alpha \log \delta^{\star}+\lambda\left(\frac{1}{1+\nu} \mathfrak{g}\left(p^{\star} ; b, k, R\right)-\frac{\nu}{1+\nu} \Psi\left(\frac{b}{\delta^{\star}}\right)\right) \Leftrightarrow \\
\mathfrak{g}\left(p^{\star} ; b, k, R\right)=\frac{(1+\nu)(1-\alpha)}{\lambda} \log \delta^{\star}+\nu \Psi\left(\frac{b}{\delta^{\star}}\right) .
\end{aligned}
$$

Mas a condição sobre $f_{Y, W}(y, w)$ garante que $\mathfrak{g}(p ; b, k, R)$ será monotônica decrescente em $p$. Assim, dado um valor de $\delta^{\star}$, o valor de $p^{\star}$, se existir, será único, e dado por

$$
p^{\star}=\mathfrak{g}^{-1}\left(\frac{(1+\nu)(1-\alpha)}{\lambda} \log \delta^{\star}+\nu \Psi\left(\frac{b}{\delta^{\star}}\right)\right) .
$$

\subsubsection{Autovalores no ponto de equilíbrio}

Sistemas dinâmicos de primeira ordem têm apenas um autovalor, o que limita o tipo de dinâmica que eles podem originar. Como o sistema dinâmico dado por (3.3.1) é de segunda ordem, ele pode ter soluções oscilatórias, estáveis ou instáveis. E, sendo não linear, pode ainda apresentar bifurcações [35, 34]. As características da resposta do sistema dinâmico podem ser obtidas dos autovalores da sua matriz jacobiana calculada no ponto de equilíbrio analisado. No caso de sistemas lineares, esses autovalores são suficientes para se obter a solução exata do sistema. Em sistemas não lineares, ela dará a aproximação do seu comportamento na vizinhança do ponto de equilíbrio.

Nesta seção, vamos então obter os autovalores da matriz jacobiana calculada no ponto de equilíbrio $\left(p^{\star}, \delta^{\star}\right)$ que foi obtido na Proposição 33, i.e.,

$$
\begin{aligned}
\delta^{\star} & =\delta^{\text {crítico }}, \mathfrak{h}_{\Psi, b}\left(\delta^{\text {crítico }}\right)=1, \\
p^{\star} & =\mathfrak{g}^{-1}\left(\frac{(1+\nu)(1-\alpha)}{\lambda} \log \delta^{\star}+\nu \Psi\left(\frac{b}{\delta^{\star}}\right)\right) .
\end{aligned}
$$

Com os autovalores, saberemos de qual tipo é o ponto de equilíbrio $\left(p^{\star}, \delta^{\star}\right)$.

Proposição 34. Os autovalores do sistema dinâmico no ponto de equilíbrio $\left(p^{\star}, \delta^{\star}\right)$, dado por (3.3.10) e (3.3.11), são dados por

$$
\gamma_{1}=\frac{\Lambda+\sqrt{\Lambda^{2}-4(\Lambda+\Xi)}}{2}
$$




$$
\gamma_{2}=\frac{\Lambda-\sqrt{\Lambda^{2}-4(\Lambda+\Xi)}}{2},
$$

com

$$
\Lambda=\alpha+\frac{b}{\delta^{\star}} \lambda \frac{\nu}{1+\nu} \psi\left(\frac{b}{\delta^{\star}}\right)
$$

$e$

$$
\Xi=-p^{\star} \delta^{\star} \mathfrak{g}^{\prime}\left(p^{\star}\right) \mathfrak{h}^{\prime}\left(\delta^{\star}\right) .
$$

Demonstração. Ver apêndice B.11.

Já sabemos quais são os autovalores da matriz jacobiana no ponto de equilíbrio $\left(p^{\star}, \delta^{\star}\right)$, o que nos permitirá saber qual é o comportamento do sistema dados os seus parâmetros. Como o sistema é discreto e não linear, sabemos que $[36,35]$

1. o ponto de equilíbrio $\left(p^{\star}, \delta^{\star}\right)$ será estável quando $\left|\gamma_{i}\right|<1, i=1,2$;

2. se os autovalores forem complexos conjugados, o sistema será subamortecido;

3. quando os autovalores são complexos conjugados com $\left|\gamma_{i}\right|<1$ e, ao modificar o valor de um parâmetro do sistema, observa-se $\left|\gamma_{i}\right|=1$, então ocorre uma bifurcação do tipo Neimark-Sacker.

Usando o resultado da Proposição 34, vamos então analisar os autovalores do ponto $\left(p^{\star}, \delta^{\star}\right)$ para verificar quando este ponto de equilíbrio é estável, e se ocorrem bifurcações.

O primeiro resultado interessante sobre a estabilidade do ponto de equilíbrio é o seguinte:

Proposição 35. Se os autovalores $\gamma_{1}$ e $\gamma_{2}$ forem reais, o ponto de equilíbrio $\left(p^{\star}, \delta^{\star}\right)$ é instável.

Demonstração. Ver apêndice B.12.

Pela Proposição 35 , sabemos então que, para que o ponto de equilíbrio $\left(p^{\star}, \delta^{\star}\right)$ seja estável, é necessário que os autovalores sejam complexos conjugados. Além disso, a condição suficiente para que este ponto seja estável é dada pela proposição a seguir:

Proposição 36. O ponto de equilíbrio $\left(p^{\star}, \delta^{\star}\right)$ é assintoticamente estável se, e somente se, $\Lambda+\Xi<1$.

Demonstração. Ver apêndice B.13. 
A Proposição 36 nos dá a condição sobre os parâmetros para que o ponto de equilíbrio não trivial seja assintoticamente estável. Além disso, lembrando também da Proposição 35, sabemos que, na condição de estabilidade, temos dois autovalores complexos conjugados dentro do círculo unitário, e isso ocorre quando $\Lambda+\Xi<1$. Assim, quando o sistema for estável, a sua resposta será sempre subamortecida. Nos exemplos de simulação do Capítulo 4, essas oscilações podem ser claramente observadas.

Além disso, lembramos que o valor de $\delta^{\star}$ é a solução da equação $\mathfrak{h}_{\Psi, b}(\delta)=1 \mathrm{e}$, portanto, depende apenas de $\Psi$ e do parâmetro $b$ dos compradores. Então, como $\Lambda=\alpha+\frac{b}{\delta^{\star}} \lambda \frac{\nu}{1+\nu} \psi\left(\frac{b}{\delta^{\star}}\right)$, observamos que quando o parâmetro $\alpha$ (memória dos vendedores) aumenta, o valor de $\Lambda$ aumenta, levando o sistema a uma condição mais próxima do limiar da estabilidade. O mesmo vale para os parâmetros $\lambda$ (sensibilidade dos vendedores) e $\nu$ (proporção entre o número de vendedores e de compradores). Obviamente, quando $\alpha=1$, i.e., os vendedores têm memória infinita, o sistema é instável, o que já era esperado.

Já a análise do parâmetro $\Xi$ é mais complicada, pois depende da forma de $\mathfrak{g}^{\prime}(p), \mathfrak{h}_{\Psi, b}^{\prime}(\delta)$ e do próprio ponto de equilíbrio $\left(p^{\star}, \delta^{\star}\right)$. Porém, podemos obter um limite mais restritivo sobre os valores dos parâmetros $\alpha, \lambda$ e $\nu$ necessário para que ocorra estabilidade, a saber:

Corolário 37. Se

$$
\alpha+\frac{b}{\delta^{\star}} \lambda \frac{\nu}{1+\nu} \psi\left(\frac{b}{\delta^{\star}}\right)>1
$$

então o ponto de equilíbrio $\left(p^{\star}, \delta^{\star}\right)$ é instável.

Demonstração. Pela Proposição 36, sabemos que $\Lambda+\Xi>1 \Rightarrow\left(p^{\star}, \delta^{\star}\right)$ é instável. Lembramos que $\Lambda=\alpha+\frac{b}{\delta^{\star}} \lambda \frac{\nu}{1+\nu} \psi\left(\frac{b}{\delta^{\star}}\right)$ e que $\Xi=-p^{\star} \delta^{\star} \mathfrak{g}^{\prime}\left(p^{\star}\right) \mathfrak{h}^{\prime}\left(\delta^{\star}\right)$. Note que $p^{\star}, \delta^{\star}>0$ e que, pela Hipótese $9, \mathfrak{g}^{\prime}(p)<0 \forall p>0$. E ainda, pela condição 2 da Proposição 33, temos que $\mathfrak{h}^{\prime}(\delta)>0 \forall \delta>0$. Portanto, $\Xi>0$ qualquer que seja o ponto $\left(p^{\star}, \delta^{\star}\right)$. Assim,

$$
\Lambda>1 \Rightarrow \Lambda+\Xi>1 \Rightarrow\left(p^{\star}, \delta^{\star}\right) \text { é instável. }
$$

Sabemos que o sistema é estável apenas quando os seus autovalores são complexos conjugados e que, ao aumentar o valor dos parâmetros $\alpha, \lambda$ e $\nu$, esses autovalores se aproximam do círculo unitário. Quando os parâmetros são tais que os autovalores se encontram sobre o círculo unitário, surge uma bifurcação de Neimark-Sacker [35]. Nesse tipo de bifurcação, que só é possível em sistemas de ordem maior ou igual a dois, o sistema passa a apresentar resposta oscilatória não amortecida estável. Esse comportamento só ocorre em sistemas não lineares: em sistemas lineares, a resposta oscilatória não amortecida que surge quando os autovalores são complexos com módulo um é sempre instável. Assim, temos o seguinte fato: 
Fato 38. Quando $\Lambda+\Xi=1$, o sistema apresenta uma bifurcação de Neimark-Sacker, e sua resposta será oscilatória não amortecida estável.

O comportamento dessas oscilações estáveis será ilustrado no Capítulo 4. No retrato de fase, ela aparece como um ciclo-limite que atrai as trajetórias iniciadas na sua vizinhança, inclusive aquelas no seu interior. É interessante notar que, no modelo para o mercado imobiliário proposto por Sommervoll et al. em [15], os resultados obtidos por simulação são dinâmicas oscilatórias não amortecidas e estáveis ${ }^{6}$.

\subsubsection{Vendedores e compradores potenciais no equilíbrio}

Na Seção 3.3.2, vimos o que acontece com o preço de mercado no equilíbrio. Agora, queremos saber o comportamento das variáveis $\boldsymbol{N}_{t}^{(A)} \mid \widetilde{\boldsymbol{P}}_{t-1}$ e $\boldsymbol{N}_{t}^{(B)} \mid \widetilde{\boldsymbol{P}}_{t-1}$. Observe que os Teoremas 19 e 24 garantem a convergência de $\boldsymbol{P}_{t} \mid \widetilde{\boldsymbol{P}}_{t-1}$ e de $\Delta_{t} \mid \widetilde{\boldsymbol{P}}_{t-1}$ quando o número de agentes aumenta. Porém, as variáveis $\boldsymbol{N}_{t}^{(A)} \mid \widetilde{\boldsymbol{P}}_{t-1}$ e $\boldsymbol{N}_{t}^{(B)} \mid \widetilde{\boldsymbol{P}}_{t-1}$ continuam aleatórias mesmo quando o número de agentes aumenta, pois suas variâncias são dadas por (2.2.12) e (2.3.4). Mas, na situação de equilíbrio, em que o sistema se converge para o seu ponto de equilíbrio $\left(p^{\star}, \delta^{\star}\right)$, obtemos um resultado sobre a distribuição dessas duas variáveis:

Proposição 39. Quando o sistema converge para o ponto de equilíbrio $\left(p^{\star}, \delta^{\star}\right)$,

$$
\mathrm{E}_{t-1}\left[\boldsymbol{N}_{t}^{(B)}\right]-\mathrm{E}_{t-1}\left[\boldsymbol{N}_{t}^{(A)}\right]=\frac{1-\alpha}{\lambda}(V+C) \log \left(\delta^{\star}\right)
$$

Demonstração. No equilíbrio, $\delta_{t}=\delta_{t-1}=\delta^{\star}$. Usando o resultado do Teorema 19, sobre a convergência de $\Delta_{t} \mid \widetilde{\boldsymbol{P}}_{t-1}$, basta aplicar o operador $\mathrm{E}_{t-1}[\cdot] \mathrm{em}(3.2 .1)$.

Corolário 40. Se $\delta^{\star}=1$, então, no equilíbrio,

$$
\mathrm{E}_{t-1}\left[\boldsymbol{N}_{t}^{(A)}\right]=\mathrm{E}_{t-1}\left[\boldsymbol{N}_{t}^{(B)}\right]
$$

Demonstração. Basta substituir $\delta^{\star}=1$ em (3.3.16).

\footnotetext{
${ }^{6}$ Os autores não obtêm resultados de convergência do processo, nem apresentam simulações do processo para diversos valores dos parâmetros, de forma que não foi possível verificar se o sistema teria outro tipo de resposta, como por exemplo convergência a um ponto de equilíbrio estável não trivial, a depender dos valores dos parâmetros.
} 


\section{Capítulo 4}

\section{Simulação}

Nos Capítulos 2 e 3, apresentamos o modelo de formação de preços no mercado imobiliário e analisamos o seu comportamento. Neste capítulo, apresentamos alguns exemplos obtidos por meio de simulações. Por meio dessas simulações, será possível verificar os resultados obtidos no Capítulo 3, além de observar os efeitos que mudanças nos parâmetros do SFH têm sobre a formação do preço de mercado.

\subsection{Distribuição de renda e riqueza}

O primeiro passo para realizar uma simulação do modelo é encontrar a distribuição conjunta de renda e riqueza dos agentes. No Brasil, a distribuição (marginal) de renda é amplamente disponível e estudada na literatura $[37,38]$. Porém, a distribuição (marginal) de riqueza, muito mais concentrada que a distribuição de renda, só foi estimada pela primeira vez por Avila em 2014 [39], usando dados da Receita Federal do Brasil, e o próprio autor do trabalho ressalta diversas limitações sobre os resultados obtidos para esta distribuição. Porém, lembramos que o nosso modelo utiliza a distribuição conjunta de renda e riqueza, que não foi ainda estimada até a elaboração deste trabalho.

Por causa desta limitação na disponibilidade da distribuição conjunta de renda e riqueza no Brasil, a simulação do modelo foi feita usando uma densidade paramétrica teórica para as variáveis $Y$ e $W$. Não foi possível usar a distribuição real porque, para o Brasil, esses dados ainda não são disponíveis.

A distribuição escolhida foi uma lognormal bivariada com a seguinte parametrização [40]:

$$
f_{Y, W}(y, w)=\frac{1}{2 \pi \sigma_{Y} \sigma_{W} \sqrt{1-\rho^{2}}} \frac{1}{y w} \exp \left[-\frac{1}{2\left(1-\rho^{2}\right)}\left(A^{2}-2 \rho A B+B^{2}\right)\right]
$$


$\operatorname{com} A=\frac{\ln y-\mu_{Y}}{\sigma_{Y}}$ e $B=\frac{\ln w-\mu_{W}}{\sigma_{W}}$.

\subsection{Vendedores}

Para a distribuição que caracteriza o comportamento dos vendedores, $\Psi$, foi usada a distribuição de Dagum com a seguinte parametrização [33]:

$$
\Psi(x \mid \alpha, \beta, \gamma)=\left[1+\left(\frac{x}{\beta}\right)^{-\alpha}\right]^{-\gamma}
$$

Essa distribuição é interessante para a simulação por diversos motivos:

1. o seu suporte é $\mathbb{R}_{+}$: como ela representa o valor pelo qual o vendedor anuncia o seu imóvel (ajustada pelo preço de mercado e pela demanda no instante anterior), valores negativos não têm sentido na realidade. Além disso, ela também atende a Hipótese 7;

2. condição da Proposição 27: no Exemplo 29, mostramos que a distribuição de Dagum atende a condição da Proposição 27 quando $\alpha \gamma>\frac{1}{b-1}$;

3. número de parâmetros: é razoável supor que a moda dessa distribuição será 1 , pois isso significa que a maior parte dos vendedores anuncia por valores próximos ao preço de mercado no instante anterior (ajustado pela percepção que eles têm sobre a demanda). Por ter três parâmetros, essa distribuição nos permite fixar o valor da moda e nos resta ainda dois graus de liberdade para obter parâmetros que atendam à condição da Proposição 27;

4. flexibilidade: a parametrização da distribuição de Dagum a torna muito flexível, produzindo ótimos ajustes a diversos tipos de conjuntos de dados.

Note que não há disponível, para a simulação que será feita neste trabalho, uma estimativa de $\Psi$ utilizando os dados de anúncios reais. Embora seja possível realizar esta estimação, ela está fora do escopo deste trabalho. Assim, a escolha dos parâmetros para realizar a simulação será feita usando duas outras hipóteses: uma sobre a moda da distribuição, e outra sobre o valor do $\delta$ no equilíbrio.

Pela construção do modelo, considerando que os valores dos anúncios dos vendedores é dado por $\boldsymbol{A}_{t}^{(i)}=\boldsymbol{a}^{(i)} \boldsymbol{P}_{t-1} \Delta_{t-1}$, é razoável supor que a maior parte dos vendedores anunciará seus imóveis por valores próximos ao preço do instante anterior. Isso equivale a supor que a moda 
da distribuição de $\boldsymbol{a}^{(i)}$ é 1, e faremos esta hipótese na simulação. Esta hipótese impõe uma condição sobre os parâmetros da distribuição $\Psi$ : como a sua moda é dada por $\beta\left(\frac{\alpha \gamma-1}{\alpha+1}\right)^{1 / \alpha}$, os parâmetros $\alpha, \beta$ e $\gamma$ devem satisfazer a equação

$$
\beta\left(\frac{\alpha \gamma-1}{\alpha+1}\right)^{1 / \alpha}=1
$$

A hipótese sobre o valor do $\delta$ no equilíbrio, junto com (4.2.1), definirá o valor dos parâmetros da distribuição $\Psi$.

\subsection{Estabilidade de preços quando $\delta^{\star}=1$}

No modelo deste trabalho, a distribuição $\Psi$ é "exógena", e precisa ser obtida com base nos dados reais dos anúncios. Porém, a sua estimativa não está disponível, e uma das possibilidades para definir os seus parâmetros para a simulação é escolher os seus valores de forma a que o equilíbrio ocorra quando $\delta=1$. Este não é o caso geral, mas nos servirá para definir os parâmetros que serão usados na simulação.

Para fazer isso, lembramos que o valor de $\delta$ no equilíbrio é um $\delta^{\star}$ que satisfaz a equação (3.3.8), i.e., $\mathfrak{h}_{\Psi, b}\left(\delta^{\star}\right)=1$. Supondo que $\delta^{\star}=1$, os parâmetros $\alpha, \beta$ e $\gamma$ devem ser tais que

$$
b-\frac{1}{\Psi(b)} \int_{0}^{b} \Psi(\xi) d \xi=1
$$

A Tabela 4.1 mostra os valores dos parâmetros usados na simulação.

Nesta primeira simulação, usamos como condição inicial do sistema

$$
\left\{\begin{array}{l}
P_{0}=85 \\
\Delta_{0}=0,9
\end{array}\right.
$$

O resultado dessa simulação está apresentado na Figura 4.3.1. Note que o preço converge para um valor um pouco abaixo de 100 , e que $\Delta_{t}$ converge para 1 , como era esperado pela forma como os parâmetros foram definidos.

Note ainda que, no equilíbrio, os processos se tornam estacionários. Além disso, observamos que as variáveis $\boldsymbol{N}_{t}^{(A)}$ e $\boldsymbol{N}_{t}^{(B)}$ ficam estacionárias (mas não degeneradas) e que, neste caso, suas esperanças são iguais. Lembrando do Corolário 40 , temos que, quando $\delta^{\star}=1$, então

$$
\mathrm{E}_{t-1}\left[\boldsymbol{N}_{t}^{(B)}\right]=\mathrm{E}_{t-1}\left[\boldsymbol{N}_{t}^{(A)}\right]
$$


Tabela 4.1: Parâmetros da simulação para equilíbrio com $\delta^{\star}=1$.

\begin{tabular}{|c|c|}
\hline \multirow{2}{*}{ vendedores } & $\begin{array}{c}V=12.000 \\
\alpha=0,6 \\
\lambda=1\end{array}$ \\
\hline & $\begin{array}{cc} & \alpha=14,549 \\
\text { distribuição } \Psi & \beta=0,9 \\
& \gamma=5,0186\end{array}$ \\
\hline compradores & $\begin{array}{c}N=120.000 \\
b=1,1\end{array}$ \\
\hline distribuição conjunta de renda e riqueza & $\begin{array}{cc}\mu_{Y}=-0,11157 & \sigma_{Y}=1,2686 \\
\mu_{W}=2,4064 & \sigma_{W}=1,0857 \\
\rho_{Y, W}=0,5\end{array}$ \\
\hline regras para obtenção de financiamento & \begin{tabular}{c}
\multicolumn{2}{c}{$R=0,6$} \\
$r=0,0072$ a.m. \\
$\tau=360$ meses $\quad k=0,033259$ \\
$\rho=30 \%$
\end{tabular} \\
\hline
\end{tabular}

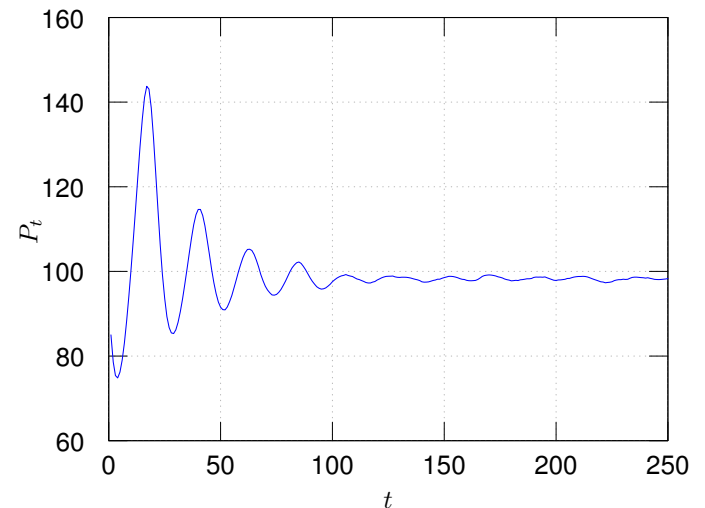

(a) Realização de $\boldsymbol{P}_{t}$

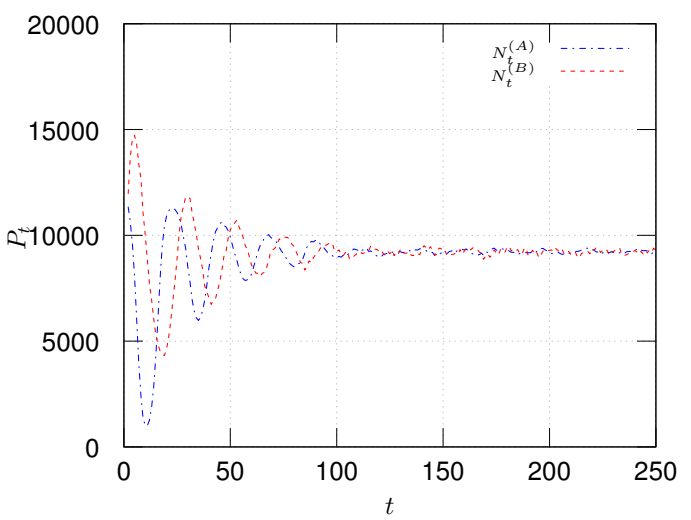

(c) Realização de $\boldsymbol{N}_{t}^{(A)}$ e de $\boldsymbol{N}_{t}^{(B)}$

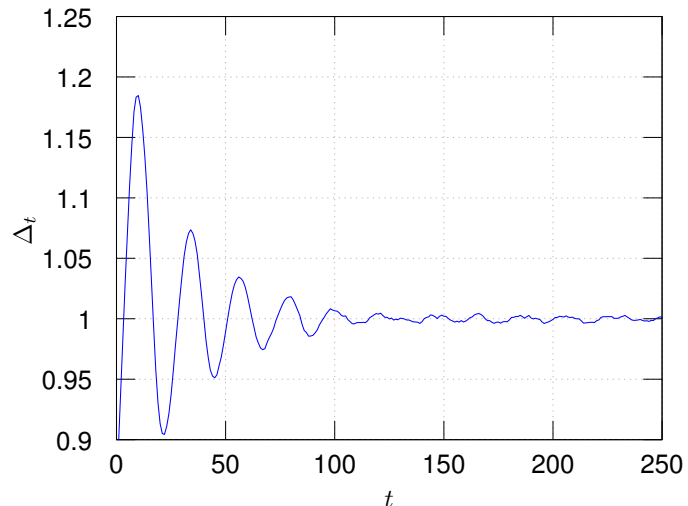

(b) Realização de $\Delta_{t}$

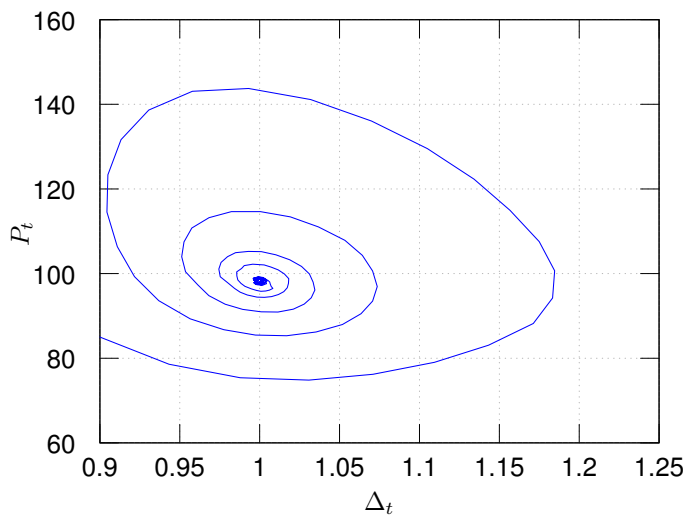

(d) $\boldsymbol{P}_{t} \times \Delta_{t}$

Figura 4.3.1: Simulação do modelo para os parâmetros da Tabela 4.1. 
E ainda, lembrando que, no equilíbrio,

$$
\mathrm{E}_{t-1}\left[\boldsymbol{N}_{t}^{(B)}\right]=C \mathfrak{g}\left(p^{\star} ; b, k, R\right)
$$

e

$$
\mathrm{E}_{t-1}\left[\boldsymbol{N}_{t}^{(A)}\right]=V \Psi\left(\frac{b}{\delta^{\star}}\right)
$$

então $\mathfrak{g}\left(p^{\star} ; b, k, R\right)=\nu \Psi\left(\frac{b}{\delta^{\star}}\right)$, como havia sido calculado em (3.3.9).

\subsection{Caso geral: estabilidade dos preços quando $\delta^{\star} \neq 1$}

Nesta seção, usamos a mesma distribuição $\Psi$ que foi usada anteriormente, i.e., $\alpha=14,549$, $\beta=0,9$ e $\gamma=5,0186$. Porém, o parâmetro dos compradores será $b=1,05$, e não 1,1 como no caso anterior. Ou seja, agora os compradores estão dispostos a pagar apenas $5 \%$ a mais do que o preço de mercado apurado no instante anterior. A equação para $\delta^{\star}$ continua sendo a mesma:

$$
\mathfrak{h}_{\Psi, b}\left(\delta^{\star}\right)=1 .
$$

Como os parâmetros da distribuição $\Psi$ são $\alpha=14,549, \beta=0,9$ e $\gamma=5,0186$ e, agora, temos $b=1,05$, a equação para $\delta^{\star}$ se reduz a

$$
b-\frac{1}{\left[1+\left(\frac{b}{\delta^{\star} \beta}\right)^{-\alpha}\right]^{-\gamma}} \int_{0}^{b}\left[1+\left(\frac{\xi}{\delta^{\star} \beta}\right)^{-\alpha}\right]^{-\gamma} d \xi=1 .
$$

Resolvendo esta equação numericamente em $\delta$, obtemos $\delta^{\star}=1,04818$. Este será o valor de $\delta$ no equilíbrio, como será verificado na simulação.

A Tabela 4.2 mostra os parâmetros que foram usados nesta simulação. O ponto inicial permanece o mesmo da simulação anterior:

$$
\left\{\begin{array}{l}
P_{0}=85 \\
\Delta_{0}=0,9
\end{array}\right.
$$

A Figura 4.4.1 mostra as realizações dos processos $\boldsymbol{P}_{t}, \Delta_{t}, \boldsymbol{N}_{t}^{(A)}$ e $\boldsymbol{N}_{t}^{(B)}$ nesse caso. De fato, observamos que os preços se estabilizam com $\mathrm{E}_{t-1}\left[\boldsymbol{N}_{t}^{(B)}\right]>\mathrm{E}_{t-1}\left[\boldsymbol{N}_{t}^{(A)}\right]$. Isso está consistente com o resultado da Proposição 39, a saber:

$$
\mathrm{E}_{t-1}\left[\boldsymbol{N}_{t}^{(B)}\right]-\mathrm{E}_{t-1}\left[\boldsymbol{N}_{t}^{(A)}\right]=\frac{1-\alpha}{\lambda}(V+C) \log \left(\delta^{\star}\right)
$$


Tabela 4.2: Parâmetros da simulação para equilíbrio com $\delta^{\star} \neq 1$.

\begin{tabular}{|c|c|}
\hline \multirow{2}{*}{ vendedores } & $\begin{array}{c}V=12.000 \\
\alpha=0,4 \\
\lambda=1\end{array}$ \\
\hline & $\begin{array}{cc} & \alpha=14,549 \\
\text { distribuição } \Psi & \beta=0,9 \\
& \gamma=5,0186\end{array}$ \\
\hline compradores & $\begin{array}{c}N=120.000 \\
b=1,05\end{array}$ \\
\hline distribuição conjunta de renda e riqueza & $\begin{array}{cc}\mu_{Y}=-0,11157 & \sigma_{Y}=1,2686 \\
\mu_{W}=2,4064 & \sigma_{W}=1,0857 \\
\rho_{Y, W}=0,5\end{array}$ \\
\hline regras para obtenção de financiamento & \begin{tabular}{c}
\multicolumn{2}{c}{$R=0,6$} \\
$r=0,0072$ a.m. \\
$\tau=360$ meses $\quad k=0,033259$ \\
$\rho=30 \%$
\end{tabular} \\
\hline
\end{tabular}

Como $\delta^{\star}>1$, então $\frac{1-\alpha}{\lambda}(V+C) \log \left(\delta^{\star}\right)>1 \Rightarrow \mathrm{E}_{t-1}\left[\boldsymbol{N}_{t}^{(B)}\right]>\mathrm{E}_{t-1}\left[\boldsymbol{N}_{t}^{(A)}\right]$.

\subsection{Memória dos vendedores e sensibilidade à demanda}

Nesta seção, vamos mostrar os resultados da simulação para valores diferentes de memória dos vendedores $(\alpha)$ e de sensibilidade à demanda $(\lambda)$. Usando os parâmetros da Tabela 4.3, simulamos o processo variando o valor de $\lambda$ e variando o valor de $\alpha$. O ponto inicial nessas simulações foi

$$
\left\{\begin{array}{l}
P_{0}=100 \\
\Delta_{0}=1
\end{array}\right.
$$

Os resultados da simulação estão apresentados nas Figuras 4.5.1 e 4.5.2.

Observe na Figura 4.5.1 que, quando a memória dos vendedores é maior, o preço de mercado ultrapassa o preço de equilíbrio antes de convergir. Este comportamento é tipicamente observado em sistemas de segunda ordem. Na realidade, este efeito pode ser interpretado da seguinte forma: ao perceber um aumento na demanda, os vendedores aumentam os preços. Porém, a sua memória maior os faz manter este comportamento por mais tempo, mesmo quando a demanda atual já diminuiu. Isso faz com que o preço de mercado aumente acima do preço de equilíbrio, para só então diminuir. 


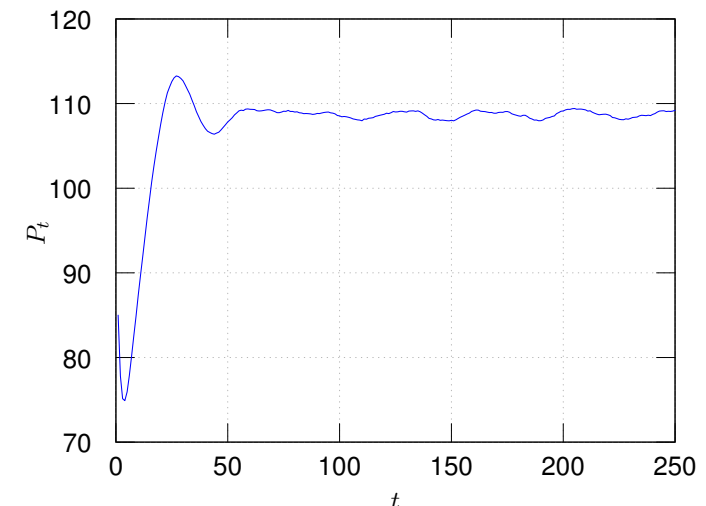

(a) Realização de $\boldsymbol{P}_{t}$

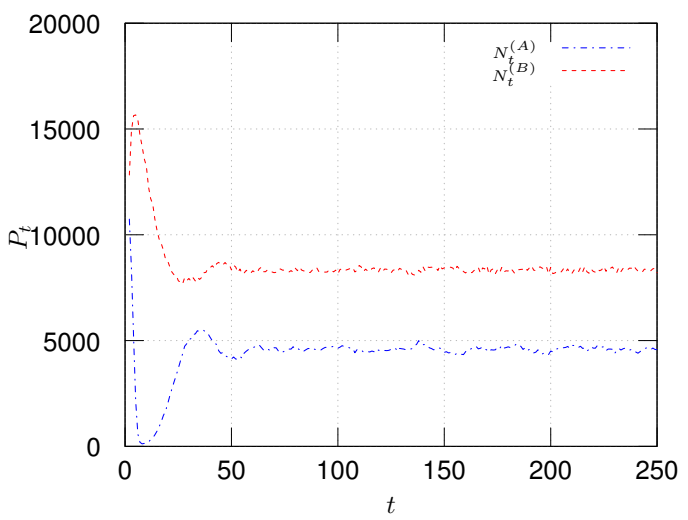

(c) Realização de $\boldsymbol{N}_{t}^{(A)}$ e de $\boldsymbol{N}_{t}^{(B)}$

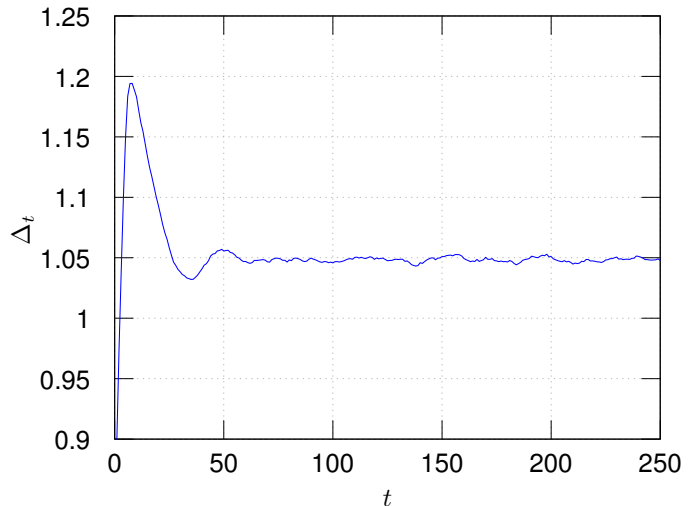

(b) Realização de $\Delta_{t}$

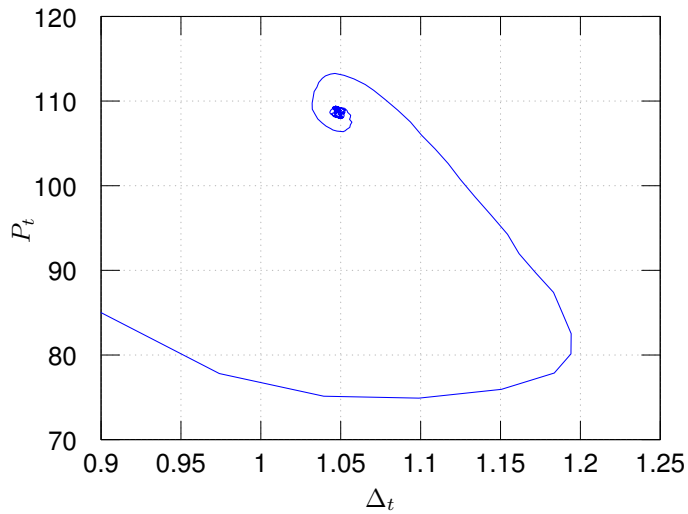

(d) $\boldsymbol{P}_{t} \times \Delta_{t}$

Figura 4.4.1: Simulação do modelo para os parâmetros da Tabela 4.2.

Tabela 4.3: Parâmetros da simulação para valores diferentes de memória de sensibilidade à demanda.

\begin{tabular}{|c|c|}
\hline \multirow[b]{2}{*}{ vendedores } & $V=12.000$ \\
\hline & $\begin{array}{cc} & \alpha=14,549 \\
\text { distribuição } \Psi & \beta=0,9 \\
& \gamma=5,0186\end{array}$ \\
\hline compradores & $\begin{array}{c}N=120.000 \\
b=1,05\end{array}$ \\
\hline distribuição conjunta de renda e riqueza & $\begin{array}{cc}\mu_{Y}=-0,11157 & \sigma_{Y}=1,2686 \\
\mu_{W}=2,4064 & \sigma_{W}=1,0857 \\
\rho_{Y, W}=0,5\end{array}$ \\
\hline regras para obtenção de financiamento & \begin{tabular}{c}
\multicolumn{2}{c}{$R=0,6$} \\
$r=0,0072$ a.m. \\
$\tau=360$ meses $\quad k=0,033259$ \\
$\rho=30 \%$
\end{tabular} \\
\hline
\end{tabular}




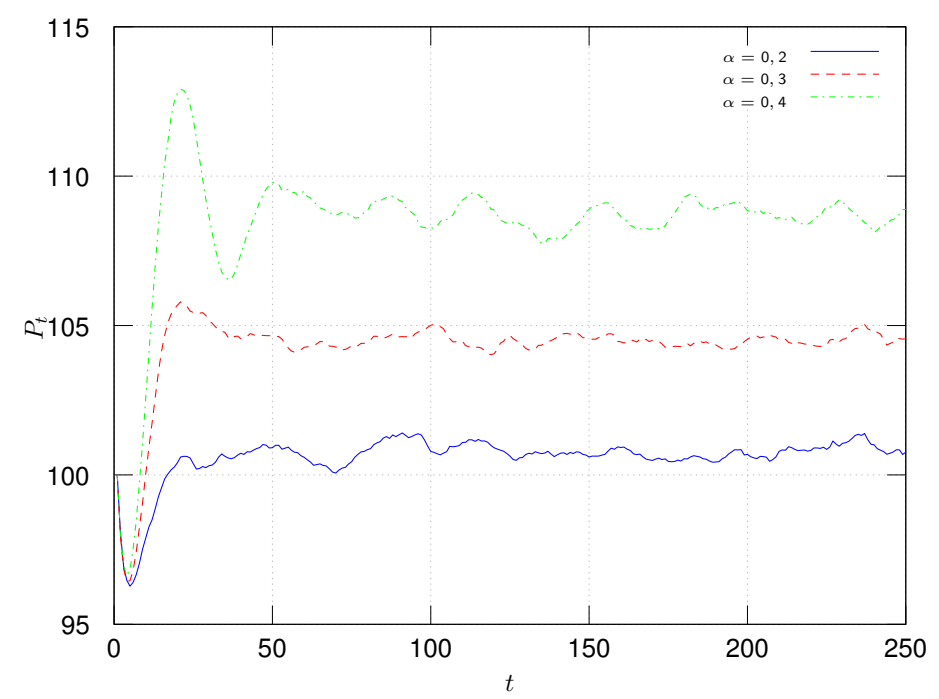

Figura 4.5.1: Simulação com $\lambda=1$ e vários valores de $\alpha$.

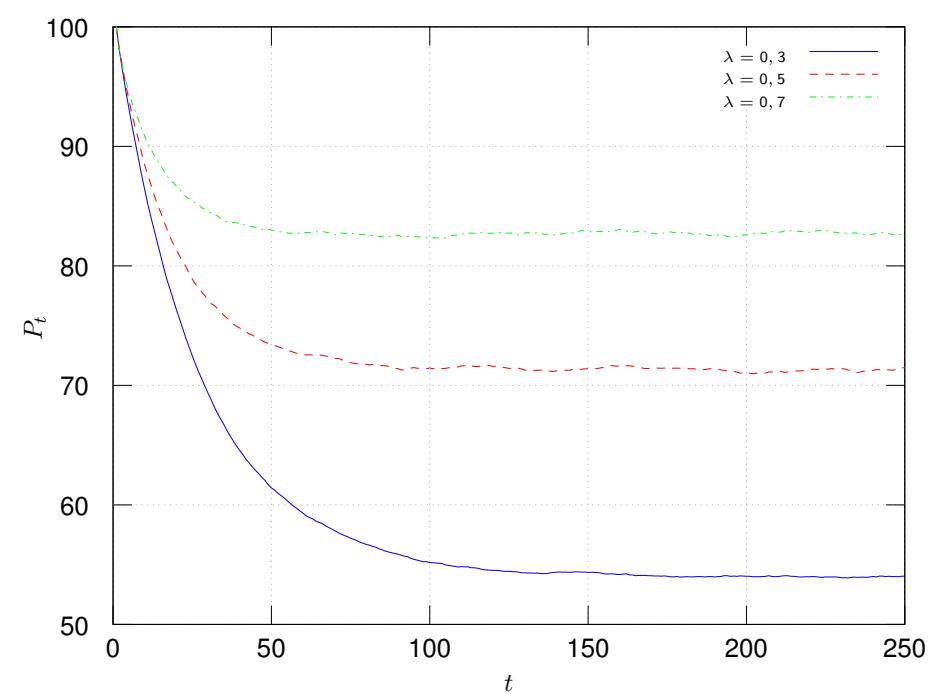

Figura 4.5.2: Simulação com $\alpha=0$ e vários valores de $\lambda$. 


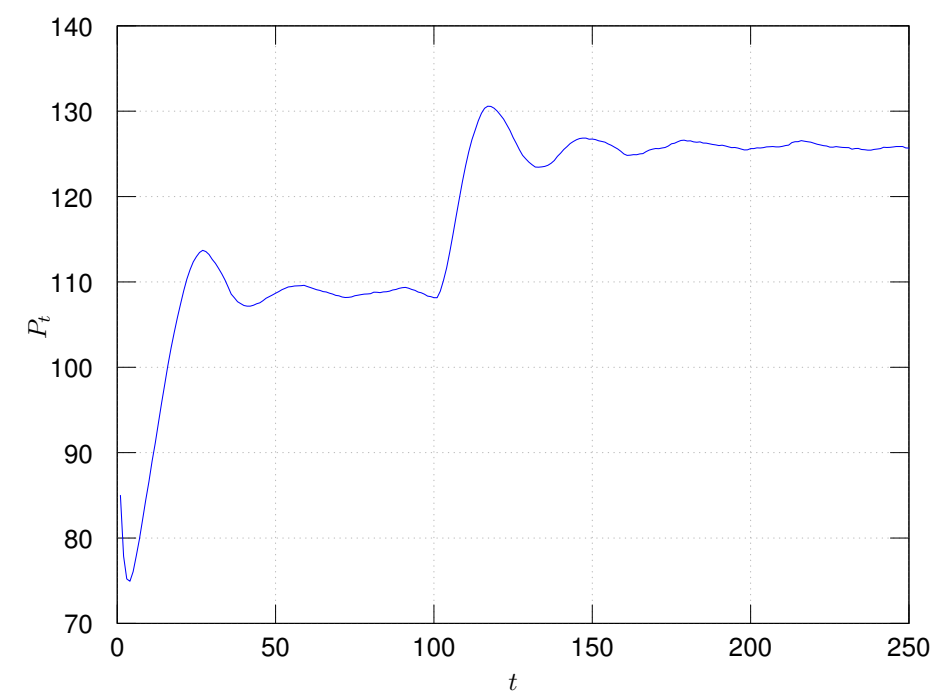

Figura 4.6.1: Efeito de uma flexibilização nas condições para obtenção de financiamento sobre o preço de mercado.

\subsection{Efeito de uma mudança nas regras de financiamento}

Quando as regras para obtenção de financiamento no SFH são modificadas, pode haver entrada ou saída de compradores potenciais do mercado. Isso porque, se as regras forem flexibilizadas, mais indivíduos conseguirão financiamento para se tornarem compradores potenciais, enquanto que se essas regras se tornarem mais severas, então menos indivíduos conseguirão financiamento.

Para verificar como o preço de mercado responde a uma mudança nessas regras, simulamos o processo da mesma forma como foi feito na Seção 4.4. Porém aqui, quando $t=100$, o LTV é aumentado de 0,6 para 0,7 . Isso cria um aumento instantâneo no número de compradores potenciais, e faz com que o preço de mercado se estabilize num novo patamar, como pode ser visto na Figura 4.6.1. Como era esperado, o novo preço de equilíbrio é maior que o anterior, consistente com o aumento de demanda criado pela flexibilização dos limites de concessão de financiamento.

\subsection{Comparação entre a aproximação $\left(p_{t}, \delta_{t}\right)$ e a realização de $\left(\boldsymbol{P}_{t}, \Delta_{t}\right)$}

Nesta seção, vamos comprar o processo $\left(\boldsymbol{P}_{t}, \Delta_{t}\right)$ com a sua aproximação determinística $\left(p_{t}, \delta_{t}\right)$ obtida no Capítulo 3. Lembramos que o sistema determinístico a que converge o 


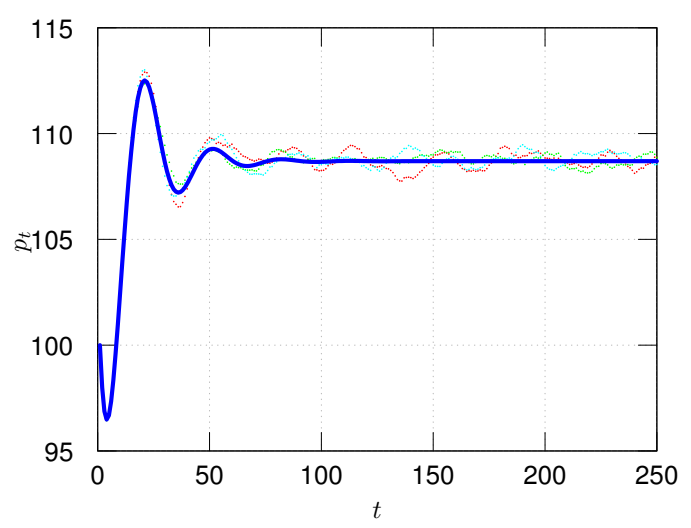

(a) $p_{t} \times t$

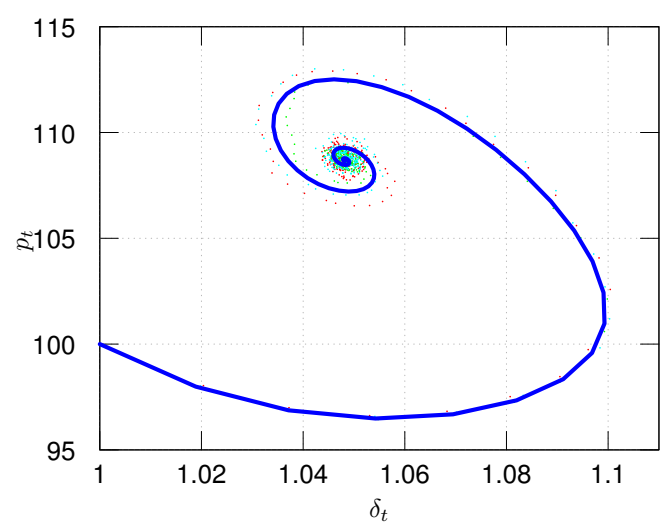

(b) $p_{t} \times \delta_{t}$

Figura 4.7.1: Comparação entre $\left(\boldsymbol{P}_{t}, \Delta_{t}\right)$ e $\left(p_{t}, \delta_{t}\right)$.

processo quando o número de agentes aumenta é descrito pelas seguintes equações:

$$
\left\{\begin{array}{l}
p_{t}=p_{t-1} \mathfrak{h}_{\Psi, b}\left(\delta_{t-1}\right) \\
\log \delta_{t}=\alpha \log \delta_{t-1}+\frac{\lambda}{1+\nu}\left[\mathfrak{g}\left(p_{t-1} ; b, k, R\right)-\nu \Psi\left(\frac{\mathfrak{b}}{\delta_{\mathfrak{t}-1}}\right)\right]
\end{array}\right.
$$

Usando os mesmos parâmetros da Tabela 4.2, $\operatorname{com} \nu=0,1$ e condição inicial

$$
\left\{\begin{array}{l}
p_{0}=100 \\
\delta_{0}=1
\end{array}\right.
$$

obtivemos os resultados da Figura 4.7.1. Para comparar com o processo, incluímos nos gráficos três realizações com a mesma parametrização e as mesmas condições iniciais.

\subsection{Bifurcação de Neimark-Sacker}

Na Seção 3.3.3, calculamos os autovalores do sistema determinístico para o qual converge o sistema no ponto de equilíbrio $\left(p^{\star}, \delta^{\star}\right)$. O valor dos autovalores é fundamentar para verificar se o ponto de equilíbrio é estável ou instável, e se ocorre algum tipo de bifurcação. Sabemos que, para alguns valores dos parâmetros, o ponto $\left(p^{\star}, \delta^{\star}\right)$ é estável e que, para outros valores, se torna instável. Além disso, quando os autovalores são tais que $\left|\gamma_{i}\right|=1, i=1,2$, ocorre uma bifurcação de Neimark-Sacker: acima desses valores, o sistema apresenta um ciclo limite estável. Nesta seção, vamos mostrar como os autovalores se modificam quando os parâmetros são alterados e como o sistema se comporta quando ocorre a bifurcação. 


$$
\begin{gathered}
\lambda=0.4- \\
\lambda=0.7- \\
\lambda=1
\end{gathered}
$$
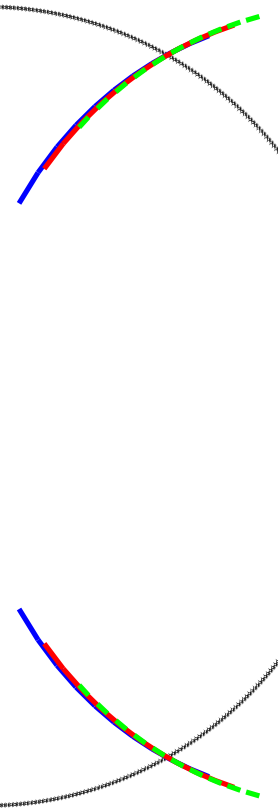

Figura 4.8.1: Root locus dos autovalores para $\alpha \in[0,1]$ e vários valores de $\lambda$.

\subsubsection{Root locus}

Primeiramente, vamos visualizar como evoluem os autovalores do sistema quando os parâmetros $\alpha$ e $\lambda$ (a memória e a sensibilidade dos vendedores) são modificados. Para isso, fizemos um root locus, que está apresentado na Figura 4.8.1.

Observe que, quando o valor de $\alpha$ aumenta, os autovalores se afastam cada vez mais da origem em direção ao círculo unitário até cruzá-lo. No cruzamento, ocorre a bifurcação de Neimark-Sacker. Valores diferentes de $\lambda$ não modificam essencialmente a forma do root locus nem o ponto onde ocorre a bifurcação, mas deslocam a curva dos autovalores. Em outras palavras, para o mesmo valor de $\alpha$, valores maiores de $\lambda$ colocam os autovalores mais próximos do círculo unitário.

\subsubsection{Dinâmica do preço e retrato de fase}

O efeito do surgimento da bifurcação é alterar o comportamento do sistema na estabilidade. Antes da bifurcação, o ponto $\left(p^{\star}, \delta^{\star}\right)$ é estável, e as trajetórias iniciadas na sua vizinhança convergem para esse ponto. Após a bifurcação, surge uma órbita estável para onde convergem as trajetórias iniciadas na sua vizinhança, incluindo aquelas iniciadas no interior do ciclo. Para ilustrar esse comportamento, simulamos o sistema com parâmetros que nos dão autovalores fora do círculo unitário. Os parâmetros gerais usados nesta simulação são aqueles da Tabela 4.3. Então, fixamos $\alpha=0,4$ e calculamos numericamente o valor $\lambda$ que nos 
dá $\left|\gamma_{i}\right|=1, i=1,2$. Note que este valor tem que ser obtido numericamente da solução da equação

$$
\Lambda+\Xi=1
$$

$\operatorname{com} \Lambda=\alpha+\frac{b}{\delta^{\star}} \lambda \frac{\nu}{1+\nu} \psi\left(\frac{b}{\delta^{\star}}\right)$ função de $\lambda$ e $\Xi=-p^{\star} \delta^{\star} \mathfrak{g}^{\prime}\left(p^{\star}\right) \mathfrak{h}^{\prime}\left(\delta^{\star}\right)$ também função de $\lambda$, pois $p^{\star}$ é função de $\lambda$. Para os parâmetros usados, o valor de $\lambda$ que nos dá $\left|\gamma_{i}\right|=1$ é

$$
\lambda^{\text {bifurc }}=1,26
$$

A Figura 4.8.2 mostra o preço para diversos valores de $\lambda$ :

- para $\lambda<\lambda^{\text {bifurc }}$ o sistema converge para o ponto de equilíbrio;

- quando $\lambda$ se aproxima de $\lambda^{\text {bifurc }}$, essa convergência ao ponto de equilíbrio vai ficando mais lenta;

- quando $\lambda=\lambda^{\text {bifurc }}$, surge um ciclo-limite, e a resposta do sistema se torna oscilatória estável em torno do ponto de equilíbrio, sem contudo convergir a ele;

- para $\lambda>\lambda^{\text {bifurc }}$, quanto maior for $\lambda$, maior é amplitude das oscilações estáveis no equilíbrio.

A Figura 4.8.3 compara o retrato de fase do sistema quando $\lambda<\lambda^{\text {bifurc }}, \lambda=\lambda^{\text {bifurc }} \mathrm{e}$ $\lambda>\lambda^{\text {bifurc }}$. Observe que, após o surgimento da bifurcação, valores maiores de $\lambda$ produzem órbitas maiores (que correspondem às amplitudes maiores observadas na Figura 4.8.2).

Finalmente, a Figura 4.8.4 ilustra como o sistema é atraído para a órbita estável após o surgimento da bifurcação, mesmo quando o sistema é iniciado num ponto no interior da órbita. O ponto $\left(p^{\star}, \delta^{\star}\right)=(115,15,1,0482)$ está marcado no gráfico com um $\times$, e os pontos iniciais das trajetórias estão marcados com um $\bullet$. 


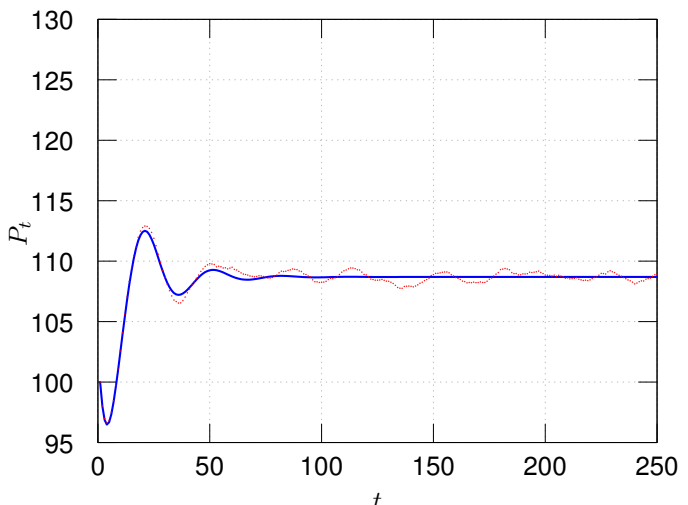

(a) $\lambda=1$

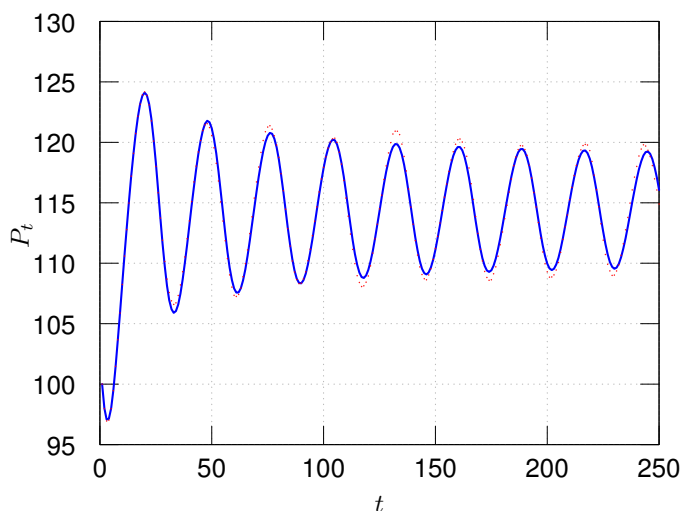

(c) $\lambda=1,26$

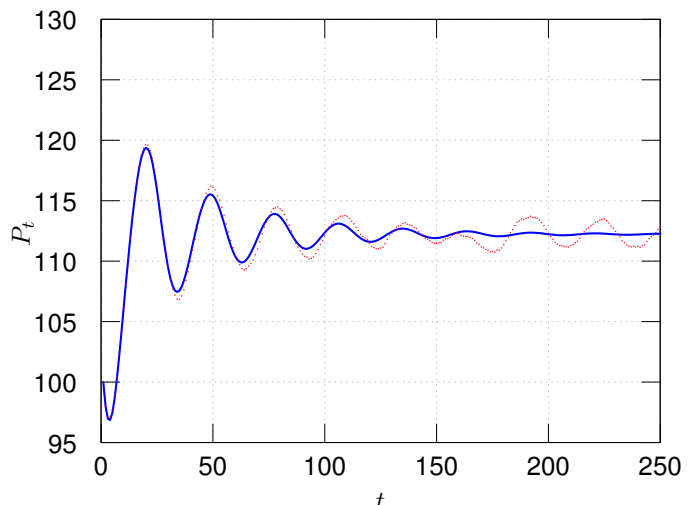

(b) $\lambda=1,15$

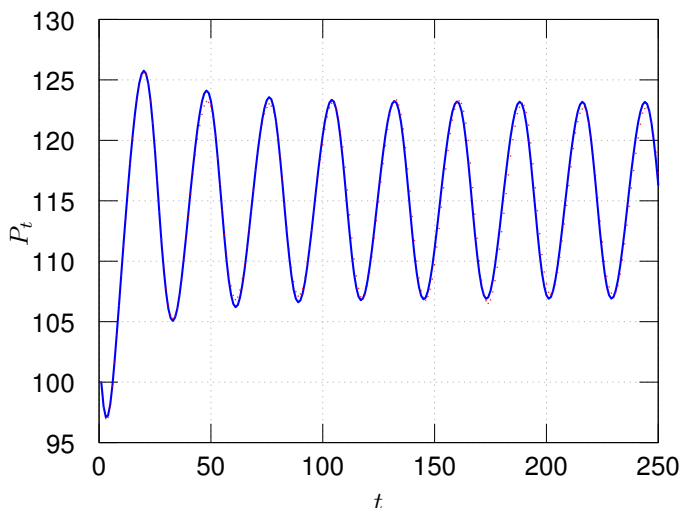

(d) $\lambda=1,3$

Figura 4.8.2: Dinâmica do preço quando ocorre a bifurcação. 


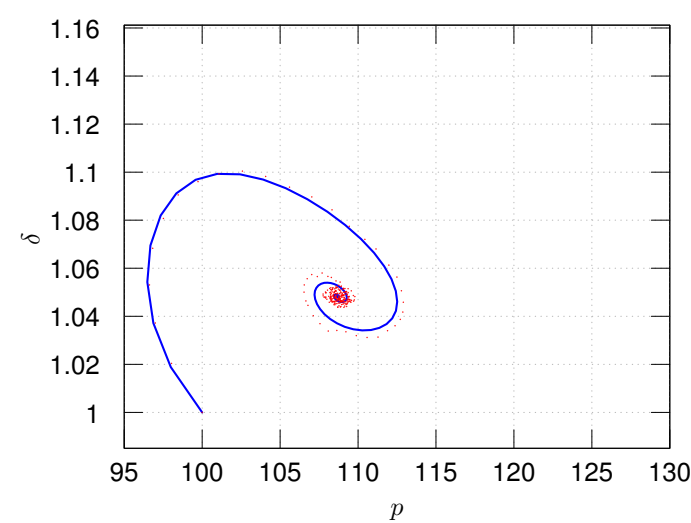

(a) $\lambda=1$

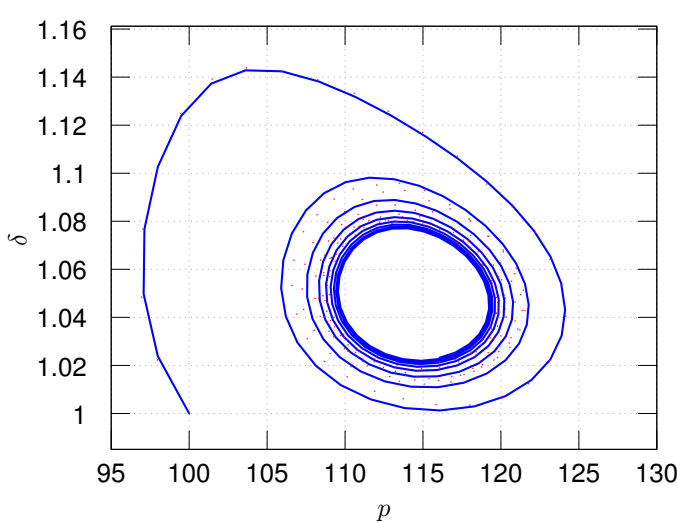

(c) $\lambda=1,26$

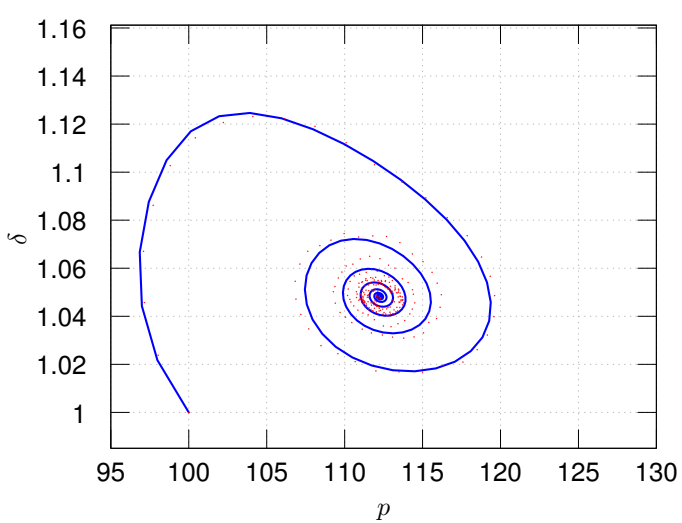

(b) $\lambda=1,15$

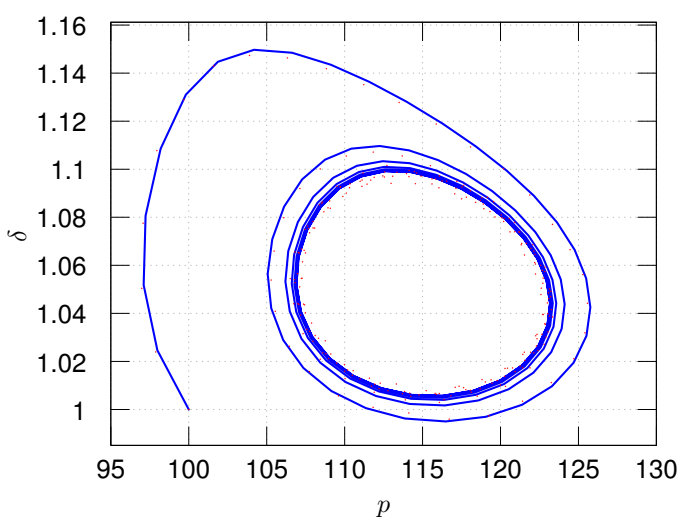

(d) $\lambda=1,3$

Figura 4.8.3: Retrato de fase quando ocorre a bifurcação.

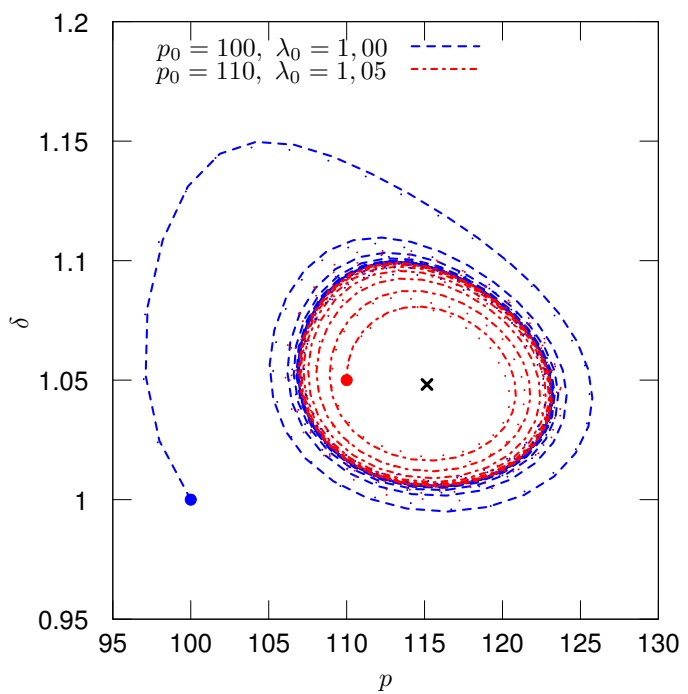

Figura 4.8.4: Órbita estável $\operatorname{com} \alpha=0,4$ e $\lambda=1,3$. 


\section{Capítulo 5}

\section{Conclusão}

Neste trabalho, construímos um modelo baseado em agentes para a formação de preços no mercado imobiliário. Diferentemente dos modelos de regressão, em que se supõe uma dinâmica conhecida para o preço e buscam-se fatores que possam "explicar" o comportamento observado da série de dados, nos modelos baseados em agentes o sistema é construído de baixo para cima, usando as características idiossincráticas dos agentes para obter o comportamento agregado do sistema.

No modelo deste trabalho, os agentes vendedores anunciam seus imóveis segundo a sua pressa, e são influenciados pelo preço e pela demanda no instante anterior. Já os agentes compradores tentam obter os recursos para entrar no mercado sujeitos às restrições impostas pelas regras de financiamento imobiliário, notadamente aquelas aplicáveis aos financiamentos no âmbito do SFH. Os compradores têm um preço de reserva comum que também depende do preço no instante anterior. Assim, a demanda por imóveis é influenciada pelas regras vigentes de financiamento, já que regras mais duras resultarão em menos compradores no mercado. Já as transações são fechadas pelo preço anunciado pelo vendedor, e não pelo preço de reserva do comprador (que é o mesmo para todos os agentes compradores). O preço de mercado é então apurado como sendo a média das transações ocorridas em cada instante.

Mostramos que o modelo assim construído resulta em um processo markoviano que converge para um sistema dinâmico não-linear de segunda ordem quando o número de agentes é grande. Essa convergência nos possibilitou obter as características da sua dinâmica em função dos parâmetros utilizados. Encontramos as condições sob as quais existe um preço de equilíbrio não trivial (i.e. diferente de zero) e obtivemos as características da dinâmica do sistema em função dos seus parâmetros. Quando o preço de equilíbrio não trivial é estável, os seus autovalores são complexos conjugados, o que leva a uma dinâmica subamortecida: durante a convergência, a resposta do sistema é oscilatória, de forma que o preço passa do valor de equilíbrio (para cima ou para baixo) até que ocorra a convergência. Além disso, 
encontramos uma bifurcação de Neimark-Sacker: quando o valor de alguns parâmetros aumenta, surge uma órbita estável em torno do ponto de equilíbrio, que atrai as trajetórias na sua vizinhança. Nesta situação, no equilíbrio o preço não converge para um valor fixo, mas oscila entre um valor máximo e um mínimo.

Pela sua construção, o modelo consegue reproduzir a dinâmica que é esperada dos preços quando ocorrem mudanças nas regras de financiamento: regras mais flexíveis resultam em aumento de preços, enquanto regras mais duras resultam em diminuição. Portanto, ele pode ser utilizado para prever o comportamento dos preços quando da mudança de tais regras. É possível calcular não só o preço de equilíbrio para o qual o sistema convergirá, mas também a trajetória que o preço seguirá até que ocorra a convergência, que pode não ser monotônica. Para isso, porém, será necessário fazer a estimação do modelo. Tanto a distribuição de renda e riqueza dos agentes (compradores) como a distribuição dos anúncios (dos vendedores) podem ser medidas. O parâmetro de preço de reserva dos compradores e a função de demanda relativa não podem ser medidos diretamente, mas foi observado no modelo que estas variáveis estão associadas, em conjunto, à definição do ponto de equilíbrio do sistema, que pode também ser medido. Embora tenha uma interpretação real, a função de demanda relativa emerge do modelo apenas como uma variável interna que dá ao sistema a sua dinâmica de ordem 2.

Em relação aos agentes compradores, o modelo utilizou a Hipótese 2, que define que os compradores só poderão entrar no mercado se conseguirem recursos suficientes para cobrir o seu preço de reserva. Ou, em outras palavras, os compradores que conseguem apenas recursos em valor menor que o seu preço de reserva são excluídos do mercado. Contudo, sabemos que, na realidade, os indivíduos que conseguem recursos em valor menor que o preço de reserva entram no mercado mesmo assim, com possibilidade de adquirir apenas imóveis mais baratos. Assim, de fato os vendedores que anunciam por valores menores têm probabilidade maior de realizarem as transações do que outros vendedores que anunciam por valores maiores. Há que se verificar, portanto, o efeito que a exclusão desses compradores tem no modelo considerando a distribuição real de renda e riqueza dos agentes. 


\section{Referências Bibliográficas}

[1] CASE, K. E.; SHILLER, R. J. The Efficiency of the Market for Single-Family Homes. [S.1.], February 1988. (Working Paper Series, 2506). Disponível em: < http: //www.nber.org/papers/w2506>.

[2] GLAESER, E. L.; GYOURKO, J. Arbitrage in Housing Markets. [S.1.], December 2007. (Working Paper Series, 13704). Disponível em: < http://www.nber.org/ papers/w13704>.

[3] GLAESER, E. L.; GYOURKO, J.; SAIZ, A. Housing Supply and Housing Bubbles. [S.1.], July 2008. (Working Paper Series, 14193). Disponível em: < http: //www.nber.org/papers/w14193>.

[4] Secretaria da Receita Federal do Brasil. Instrução Normativa 84. 2001.

[5] BRASIL. Lei 11.196. 2005. E alterações posteriores.

[6] Colégio Notarial do Brasil - Seção São Paulo. Emolumentos. 2014. Disponível em: < http://www.irib.org.br/arquivos/emolumentos/ Sao_Paulo_Custas_e_Emolumentos_2014.pdf>.

[7] NETTO, C. R. L. Condicionantes de preços dos imóveis residenciais nos municípios de São Paulo e Rio de Janeiro e a possibilidade de formação de bolhas imobiliárias. Dissertação (Mestrado) - Universidade de São Paulo, Faculdade de Economia, Administração e Contabilidade, junho 2013. Disponível em: < http://www.teses.usp.br/teses/disponiveis/12/12136/tde12082013-182331/publico/CassioRobertoLeiteNettoVC.pdf>.

[8] SINAI, T.; SOULELES, N. S. Owner-Occupied Housing as a Hedge Against Rent Risk. [S.1.], January 2003. (Working Paper Series, 9462). Disponível em: < http: //www.nber.org/papers/w9462>.

[9] STEIN, J. C. Prices and Trading Volume in the Housing Market: A Model with Downpayment Effects. [S.1.], March 1993. (Working Paper Series, 4373). Disponível em: < http: / / www.nber.org/papers/w4373>. 
[10] ORTALO-MAGNE, F.; RADY, S. Housing market dynamics: On the contribution of income shocks and credit constraints. The Review of Economic Studies, Oxford University Press, v. 73, n. 2, p. 459-485, 2006.

[11] NETTO, C. R. L.; FÁVERO, L. P. L.; SUZART, J. A. D. S. House prices and real estate bubbles in brazil: an analysis through johansen cointegration. International Journal of Applied Decision Sciences, Inderscience Publishers (IEL), v. 8, n. 4, p. 339-357, 2015.

[12] DIECI, R.; WESTERHOFF, F. Modeling house price dynamics with heterogeneous speculators. Global Analysis of Dynamic Models in Economics and Finance, Springer, p. 35.

[13] BOLT, W. et al. Identifying booms and busts in house prices under heterogeneous expectations. De Nederlandsche Bank Working Paper, 2014.

[14] EICHHOLTZ, P.; HUISMAN, R.; ZWINKELS, R. C. Fundamentals or trends? a longterm perspective on house prices. Applied Economics, Taylor \& Francis, v. 47, n. 10, p. 1050-1059, 2015.

[15] SOMMERVOLL, D. E.; BORGERSEN, T.-A.; WENNEMO, T. Endogenous housing market cycles. Journal of Banking \& Finance, v. 34, n. 3, p. 557 - 567, 2010. ISSN 0378-4266. Disponível em: < http://www.sciencedirect.com/ science/article/pii/S0378426609002271>.

[16] SILVA, M. A. da. Livro de ofertas e dinâmica de preços: evidências a partir de dados da BOVESPA. Dissertação (Mestrado) - Universidade de São Paulo, Escola de Artes Ciências e Humanidades, 2013.

[17] BRASIL. Relatório de Estabilidade Financeira. [S.1.], março 2015. Disponível em: < http: / / www.bcb.gov.br/?RELESTAB201503>.

[18] Banco Central do Brasil. Circular 3.671. 2013.

[19] Câmara dos Deputados. Poupança: a história do mais tradicional investimento do país. 6 2012. Disponível em: < http://www2.camara.leg.br/ camaranoticias/radio/materias/REPORTAGEM-ESPECIAL/420768POUPANCA-A-HISTORIA-DO-MAIS-TRADICIONAL-INVESTIMENTO-DOPAIS-BLOCO-2.html>.

[20] Conselho Monetário Nacional. Resolução 1.980. 1993. E alterações posteriores.

[21] Conselho Monetário Nacional. Resolução 3.932. 2010. E alterações posteriores até agosto de 2015 .

[22] BRASIL. Lei 8.692. 1993. E alterações posteriores. 
[23] CAMPBELL, T. S.; DIETRICH, J. K. The determinants of default on insured conventional residential mortgage loans. The Journal of Finance, Wiley Online Library, v. 38, n. 5 , p. $1569-1581,1983$.

[24] QUIGLEY, J. M.; ORDER, R. V. Defaults on mortgage obligations and capital requirements for us savings institutions: A policy perspective. Journal of Public Economics, Elsevier, v. 44, n. 3, p. 353-369, 1991.

[25] WONG, J. et al. Residential mortgage default risk and the loan-to-value ratio. Hong Kong monetary authority quarterly bulletin, p. 35-45, 2004.

[26] QI, M.; YANG, X. Loss given default of high loan-to-value residential mortgages. Journal of Banking \& Finance, Elsevier, v. 33, n. 5, p. 788-799, 2009.

[27] BRASIL. Lei 8.036. 1990. E alterações posteriores.

[28] BRASIL. Lei 9.514. 1997. E alterações posteriores.

[29] ROSS, S. A first course in probability. 5th. ed. [S.1.]: Prentice-Hall, 1997.

[30] LIMA, E. L. Curso de análise. 12. ed. [S.1.]: Instituto de Matemática Pura e Aplicada, 2008. (Projeto Euclides, v. 1). ISBN 9788524401183.

[31] PRADO, F. P. de A. Fenômenos críticos em sistemas de partículas interagentes e suas aplicações na modelagem de mercados financeiros. Tese (Doutorado) — Universidade de São Paulo, Instituto de Matemática e Estatísica, 2004.

[32] DAWID, P. E. Ergodicidade em sistemas econômicos com interação social e agentes heterogêneos. Tese (Doutorado) - Universidade de São Paulo, Instituto de Matemática e Estatísica, 2009.

[33] KLEIBER, C. A guide to the dagum distributions. 2008.

[34] ATTUX, R. R. de F. Sobre dinâmica caótica e convergência em algoritmos de equalização autodidata. Dissertação (Mestrado) - Universidade Estadual de Campinas, Faculdade de Engenharia Elétrica e de Computação, 2001.

[35] KUZNETSOV, Y. A. Elements of applied bifurcation theory. [S.1.]: Springer Science \& Business Media, 2013.

[36] GEROMEL, J.; PALHARES, A. Análise Linear de Sistemas Dinâmicos: Teoria, Ensaios Práticos e Exercıcios. [S.1.]: Blucher, Sao Paulo, SP, 2004.

[37] ELBERS, C.; LANJOUW, J. O. Poverty and inequality in brazil: New estimates from combined ppv-pnad. Inequality and economic development in Brazil, World Bank Publications, p. 81, 2004. 
[38] HOFFMANN, R. Distribuição de renda e crescimento econômico. Estudos Avançados, v. 15 , n. 41 , p. 67-76, 2001.

[39] CASTRO, F. A. de. Imposto de renda da pessoa física: comparações internacionais, medidas de progressividade e redistribuição. Dissertação (Mestrado) — Universidade de Brasília, Faculdade de economia, administração e contabilidade, 2014.

[40] MOSTAFA, M.; MAHMOUD, M. On the problem of estimation for the bivariate lognormal distribution. Biometrika, JSTOR, p. 522-527, 1964.

[41] BRASIL. Lei 8.177. 1991. E alterações posteriores.

[42] Banco Central do Brasil. Circular 3.093. 2002. E alterações posteriores.

[43] Banco Central do Brasil. Circular 3.655. 2013. E alterações posteriores.

[44] JAMES, B. R. Probabilidade: um curso em nível intermediário. 2. ed. [S.1.]: Instituto de Matemática Pura e Aplicada, 2009. (Projeto Euclides). ISBN 9788524401015.

[45] CHAO, W. E. S. M. T. Negative moments of positive random variables. Journal of the American Statistical Association, Taylor \& Francis, Ltd., v. 67, n. 338, p. 429-431, 1972. ISSN 01621459. Disponível em: <http://www.jstor.org/stable/2284399>. 


\section{Apêndice A}

\section{Poupança}

\section{A.1 Taxa de remuneração}

A poupança teve a sua remuneração fixada em $6 \%$ a.a. em 1861 pelo decreto de Dom Pedro II que criou a CEF. Até 2012, a taxa de remuneração dos depósitos da poupança permaneceu em $0,5 \%$ a.m. + TR, com rendimentos isentos de imposto de renda. A taxa referencial (TR) é um indexador da poupança definido na Lei 8.177/91 [41], que fica próximo ou é igual a zero (ver Figura A.1.1). Ao longo dos anos 1990 e 2000, a taxa de juros livre de risco no Brasil foi muito mais alta que a taxa de remuneração da poupança (ver Figura A.1.1), de forma que este investimento era utilizado apenas por pequenos aplicadores. Até que, em 2012, a taxa livre de risco caiu a patamares históricos muito baixos, e a taxa de remuneração da poupança, sobre a qual não incide imposto de renda, tornou-se atraente também para investidores maiores, como fundos investimento. Para evitar que a dívida pública migrasse para a poupança, criando distorções no mercado, em 2012 foi publicada a Lei 12.703, que alterou o art. 12, II da Lei 8.177/91, definindo uma nova a regra de remuneração mensal dos depósitos de poupança:

$$
\left\{\begin{array}{ll}
0,5 \%+\mathrm{TR}, & \text { se taxa Selic }>8,5 \% \text { a.a. } \\
70 \% \text { taxa Selic }+ \text { TR, }, & \text { caso contrário }
\end{array} .\right.
$$

Com essa alteração, conseguiu-se evitar que a poupança se tornasse uma opção atraente para grandes investidores mesmo quando a taxa Selic ficasse em valores baixos, mantendo o benefício de isenção de imposto de renda sobre os rendimentos da poupança. 


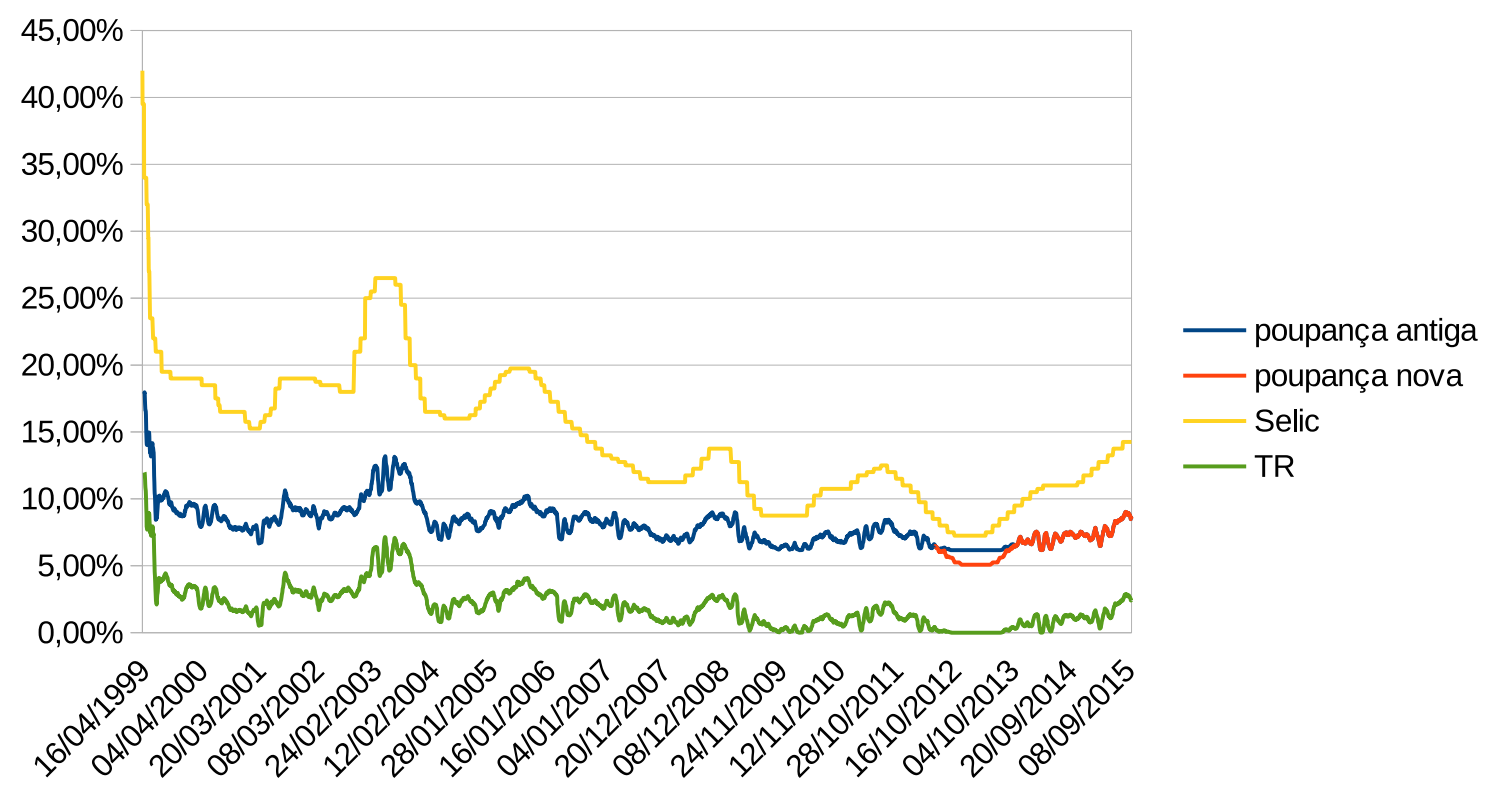

Figura A.1.1: Taxa Selic, TR e remuneração da poupança.

\section{A.2 Encaixe compulsório e direcionamento obrigatório}

Os depósitos, tanto à vista quanto a prazo, estão sujeitos a encaixe compulsório no $\mathrm{BCB}$, ou seja, a cada real captado pela IF, alguns centavos devem ser recolhidos ao BCB e não podem ser usados para realizar operações. O encaixe compulsório é o mecanismo que permite ao BCB determinar qual será o multiplicador bancário na economia e é, portanto, um importante instrumento de política monetária. Cada tipo de depósito tem regras específicas de encaixe compulsório, e essas regras são modificadas constantemente para acomodar os objetivos de política monetária definidos pelo governo.

No caso da poupança, em agosto de 2015 , o art. $1^{\circ}$ do regulamento anexo à Resolução CMN 3.932/10 define que 24,5\% dos recursos captados devem ser recolhidos em encaixe compulsório ao $\mathrm{BCB}$. Esse encaixe é remunerado à taxa definida no art. $7^{\circ}$ da Circular $\mathrm{BCB}$ 3.093/02 [42]:

$$
\left\{\begin{array}{ll}
0,617 \%+\mathrm{TR}, & \text { se taxa Selic }>8,5 \% \text { a.a. } \\
70 \% \text { taxa Selic }+ \text { TR, } & \text { caso contrário. }
\end{array} .\right.
$$

Note que, quando a taxa Selic está acima de 8,5\% a.a., essa remuneração é um pouco maior que a taxa de captação, de $0,5 \%+$ TR.

Além desse encaixe, a resolução determina um encaixe adicional de $5,5 \%$ das captações, que é remunerado à taxa Selic [43], muito maior que o custo da captação (em 18 de setembro de 
2015, o custo de captação da poupança era de apenas 8, $57 \%$ a.a., contra $14,25 \%$ a.a. da taxa Selic).

Apenas $5 \%$ dos recursos captados por poupança podem ser aplicados livremente pela IF. O restante, i.e., $65 \%$ dos recursos, deve ser aplicado em financiamentos imobiliários. Desses $65 \%$, no mínimo $80 \%$ deve ser aplicado em financiamentos no âmbito do SFH e os $20 \%$ restantes podem ser aplicados em financiamentos imobiliários a taxas livres.

A Resolução CMN 3.932/2010 permite também que o direcionamento obrigatório dos recursos da poupança seja cumprido com outros tipos de ativos além dos financiamentos imobiliários. Os ativos que são considerados equivalentes aos financiamentos imobiliários no âmbito do SFH para fins de cumprimento da exigibilidade de direcionamento obrigatório da poupança estão listados no art. $2^{\circ}$ do Regulamento Anexo à Resolução CMN 3.932/2010, e os ativos que são considerados equivalentes aos financiamentos imobiliários a taxas livres para fins de cumprimento da exigibilidade estão listados no art. $3^{\circ}$. A principal dessas equivalências são os depósitos interfinanceiros imobiliários, cujo objetivo é permitir que instituições que optem por não realizar operações de financiamento imobiliário disponibilizem os recursos para que a CEF (ou outra IF integrante do SBPE interessada) possa realizar as operações.

Finalmente, em 2015, os recursos da poupança disponíveis no sistema financeiro para realizar operações de financiamento imobiliário tornaram-se escassos, por diversos motivos que não serão discutidos aqui. Para aumentar um pouco a disponibilidade de recursos disponíveis para o financiamento imobiliário, a Circular BCB 3.757/2015 permitiu utilizar até $18 \%$ do encaixe compulsório de $24,5 \%$ dos depósitos de poupança em novas operações de financiamento imobiliário no âmbito do SFH. 


\section{Apêndice B}

\section{Demonstrações}

\section{B.1 Demonstração do Fato 13}

Para obter essa expressão, basta notar que

$$
\begin{aligned}
\mathbb{P}_{t-1}\left(\boldsymbol{n}_{t}>n\right) & =\mathbb{P}_{t-1}\left(\min \left\{\boldsymbol{N}_{t}^{(A)}, \boldsymbol{N}_{t}^{(B)}\right\}>n\right) \\
& =\mathbb{P}_{t-1}\left(\boldsymbol{N}_{t}^{(A)}>n, \boldsymbol{N}_{t}^{(B)}>n\right)
\end{aligned}
$$

Como, pela Hipótese 4, as variáveis $\boldsymbol{N}_{t}^{(A)}$ e $\boldsymbol{N}_{t}^{(B)}$ são condicionalmente independentes dado o passado, então (B.1.1) se reduz a

$$
\mathbb{P}_{t-1}\left(\boldsymbol{n}_{t}>n\right)=\mathbb{P}_{t-1}\left(\boldsymbol{N}_{t}^{(A)}>n\right) \mathbb{P}_{t-1}\left(\boldsymbol{N}_{t}^{(B)}>n\right)
$$

Então,

$$
\begin{aligned}
\mathbb{P}_{t-1}\left(\boldsymbol{n}_{t} \leq n\right) & =1-\mathbb{P}_{t-1}\left(\boldsymbol{n}_{t}>n\right) \\
= & 1-\mathbb{P}_{t-1}\left(\boldsymbol{N}_{t}^{(A)}>n\right) \mathbb{P}_{t-1}\left(\boldsymbol{N}_{t}^{(B)}>n\right) \\
= & \mathbb{P}_{t-1}\left(\boldsymbol{N}_{t}^{(A)} \leq n\right)+\mathbb{P}_{t-1}\left(\boldsymbol{N}_{t}^{(B)} \leq n\right)-\mathbb{P}_{t-1}\left(\boldsymbol{N}_{t}^{(A)} \leq n\right) \mathbb{P}_{t-1}\left(\boldsymbol{N}_{t}^{(B)} \leq n\right)
\end{aligned}
$$


Finalmente, calculamos $\mathbb{P}_{t-1}\left(\boldsymbol{n}_{t}=n\right)=\mathbb{P}_{t-1}\left(\boldsymbol{n}_{t} \leq n\right)-\mathbb{P}_{t-1}\left(\boldsymbol{n}_{t} \leq n-1\right)$ usando (B.1.2) para obter, após alguma álgebra,

$$
\begin{aligned}
\mathbb{P}_{t-1}\left(\boldsymbol{n}_{t}=n\right)=\mathbb{P}_{t-1}( & \left.\boldsymbol{N}_{t}^{(A)}=n\right)+\mathbb{P}_{t-1}\left(\boldsymbol{N}_{t}^{(B)}=n\right) \\
-\mathbb{P}_{t-1}\left(\boldsymbol{N}_{t}^{(A)}=n\right) \mathbb{P}_{t-1}\left(\boldsymbol{N}_{t}^{(B)} \leq n\right) & -\mathbb{P}_{t-1}\left(\boldsymbol{N}_{t}^{(B)}=n\right) \mathbb{P}_{t-1}\left(\boldsymbol{N}_{t}^{(A)} \leq n-1\right) .
\end{aligned}
$$

\section{B.2 Demonstração da Proposição 15}

Para calcular a distribuição expressa em (2.5.3), observamos que há duas possibilidades para o numerador:

1. $x>b \boldsymbol{P}_{t-1} \Longrightarrow\left(\boldsymbol{A}_{t}^{(i)} \leq p\right) \cap\left(\boldsymbol{A}_{t}^{(i)} \leq b \boldsymbol{P}_{t-1}\right) \equiv \boldsymbol{A}_{t}^{(i)} \leq b \boldsymbol{P}_{t-1}$ :

Neste caso, temos

$$
\mathbb{P}_{t-1}\left(\boldsymbol{T}_{t}^{(k)} \leq x\right)=\frac{\mathbb{P}_{t-1}\left(\boldsymbol{A}_{t}^{(i)} \leq b \boldsymbol{P}_{t-1}\right)}{\mathbb{P}_{t-1}\left(\boldsymbol{A}_{t}^{(i)} \leq b \boldsymbol{P}_{t-1}\right)}=1
$$

2. $x \leq b \boldsymbol{P}_{t-1} \Longrightarrow\left(\boldsymbol{A}_{t}^{(i)} \leq p\right) \cap\left(\boldsymbol{A}_{t}^{(i)} \leq b \boldsymbol{P}_{t-1}\right) \equiv \boldsymbol{A}_{t}^{(i)} \leq x$ :

Neste caso, usando (2.3.2), temos

$$
\begin{aligned}
\mathbb{P}_{t-1}\left(\boldsymbol{T}_{t}^{(k)} \leq x\right) & =\frac{\mathbb{P}_{t-1}\left(\boldsymbol{A}_{t}^{(i)} \leq x\right)}{\mathbb{P}_{t-1}\left(\boldsymbol{A}_{t}^{(i)} \leq b \boldsymbol{P}_{t-1}\right)} \\
& =\frac{\Psi\left(\frac{x}{\boldsymbol{P}_{t-1} \Delta_{t-1}}\right)}{\Psi\left(\frac{b \boldsymbol{P}_{t-1}}{\boldsymbol{P}_{t-1} \Delta_{t-1}}\right)}=\frac{\Psi\left(\frac{x}{\boldsymbol{P}_{t-1} \Delta_{t-1}}\right)}{\Psi\left(\frac{b}{\Delta_{t-1}}\right)}
\end{aligned}
$$

Assim, obtemos a seguinte distribuição condicional de $\boldsymbol{T}_{t}^{(k)}$ :

$$
\mathbb{P}_{t-1}\left(\boldsymbol{T}_{t}^{(k)} \leq x\right)= \begin{cases}1, & x>b \boldsymbol{P}_{t-1} \\ \frac{\Psi\left(\frac{x}{P_{t-1} \Delta_{t-1}}\right)}{\Psi\left(\frac{b}{\Delta_{t-1}}\right)}, & x \leq b \boldsymbol{P}_{t-1}\end{cases}
$$




\section{B.3 Demonstração do Lema 18}

Primeiro, vamos calcular $\mu_{t}:=\mathrm{E}\left[\log \Delta_{t} \mid \boldsymbol{P}_{t-1}, \Delta_{t-1}\right]$. Lembrando que $\log \Delta_{t}$ é dado por (3.2.1), temos que

$$
\begin{aligned}
\mu_{t} & =\mathrm{E}\left[\alpha \log \Delta_{t-1}+\frac{\lambda}{V+C}\left(\boldsymbol{N}_{t}^{(B)}-\boldsymbol{N}_{t}^{(A)}\right) \mid \boldsymbol{P}_{t-1}, \Delta_{t-1}\right] \\
= & \alpha \log \Delta_{t-1}+ \\
& +\frac{\lambda}{V+C}\left(\mathrm{E}\left[\boldsymbol{N}_{t}^{(B)} \mid \boldsymbol{P}_{t-1}, \Delta_{t-1}\right]-\mathrm{E}\left[\boldsymbol{N}_{t}^{(A)} \mid \boldsymbol{P}_{t-1}, \Delta_{t-1}\right]\right) .
\end{aligned}
$$

Substituindo (2.2.11) e (2.3.3) em (B.3.1), temos

$$
\mu_{t}=\alpha \log \Delta_{t-1}+\frac{\lambda}{V+C}\left(C \mathfrak{g}_{t-1}-V \Psi\left(\frac{b}{\Delta_{t-1}}\right)\right) .
$$

Finalmente, substituindo (3.2.2) em (B.3.2), temos

$$
\mu_{t}=\alpha \log \Delta_{t-1}+\lambda\left(\frac{1}{1+\nu} \mathfrak{g}_{t-1}-\frac{\nu}{1+\nu} \Psi\left(\frac{b}{\Delta_{t-1}}\right)\right)
$$

Agora, vamos calcular $\sigma_{t}^{2}:=\operatorname{Var}\left(\log \Delta_{t} \mid \boldsymbol{P}_{t-1}, \Delta_{t-1}\right)$ :

$$
\begin{aligned}
\sigma_{t}^{2} & =\operatorname{Var}\left(\alpha \log \Delta_{t-1}+\frac{\lambda}{V+C}\left(\boldsymbol{N}_{t}^{(B)}-\boldsymbol{N}_{t}^{(A)}\right) \mid \boldsymbol{P}_{t-1}, \Delta_{t-1}\right) \\
& =\left(\frac{\lambda}{V+C}\right)^{2} \operatorname{Var}\left(\boldsymbol{N}_{t}^{(B)}-\boldsymbol{N}_{t}^{(A)} \mid \boldsymbol{P}_{t-1}, \Delta_{t-1}\right)
\end{aligned}
$$

Lembramos que as variáveis $\boldsymbol{N}_{t}^{(B)}$ e $\boldsymbol{N}_{t}^{(A)}$ não são independentes mas, pela Hipótese $4, \boldsymbol{N}_{t}^{(B)}$ e $\boldsymbol{N}_{t}^{(A)}$ são condicionalmente independentes dado o passado. Usando este fato em (B.3.3), temos

$$
\sigma_{t}^{2}=\left(\frac{\lambda}{V+C}\right)^{2}\left[\operatorname{Var}\left(\boldsymbol{N}_{t}^{(B)} \mid \boldsymbol{P}_{t-1}, \Delta_{t-1}\right)+\operatorname{Var}\left(\boldsymbol{N}_{t}^{(A)} \mid \boldsymbol{P}_{t-1}, \Delta_{t-1}\right)\right]
$$

Substituindo (2.2.12) e (2.3.4) em (B.3.4), temos

$$
\sigma_{t}^{2}=\frac{\lambda^{2}}{(V+C)^{2}}\left[C \mathfrak{g}_{t-1}\left(1-\mathfrak{g}_{t-1}\right)+V \Psi\left(\frac{b}{\Delta_{t-1}}\right)\left(1-\Psi\left(\frac{b}{\Delta_{t-1}}\right)\right)\right]
$$


E, finalmente, substituindo (3.2.2) em (B.3.5), temos

$$
\sigma_{t}^{2}=\frac{\lambda^{2}}{V+C}\left[\frac{1}{1+\nu} \mathfrak{g}_{t-1}\left(1-\mathfrak{g}_{t-1}\right)+\frac{\nu}{1+\nu} \Psi\left(\frac{b}{\Delta_{t-1}}\right)\left(1-\Psi\left(\frac{b}{\Delta_{t-1}}\right)\right)\right]
$$

\section{B.4 Demonstração do Lema 20}

Usando a propriedade básica da esperança condicional ${ }^{1}$ [44], temos

$$
\begin{aligned}
\mathrm{E}_{t-1}\left[\boldsymbol{P}_{t}\right] & =\mathrm{E}_{t-1}\left\{\mathrm{E}_{t-1}\left[\boldsymbol{P}_{t} \mid \boldsymbol{n}_{t}\right]\right\} \\
& =\sum_{n=0}^{\infty} \mathrm{E}_{t-1}\left[\boldsymbol{P}_{t} \mid \boldsymbol{n}_{t}=n\right] \mathbb{P}\left(\boldsymbol{n}_{t}=n\right) \\
& =\sum_{n=0}^{\infty} \mathrm{E}_{t-1}\left[\frac{1}{\boldsymbol{n}_{t}} \sum_{k=1}^{\boldsymbol{n}_{t}} \boldsymbol{T}_{t}^{(k)} \mid \boldsymbol{n}_{t}=n\right] \mathbb{P}\left(\boldsymbol{n}_{t}=n\right) \\
& =\sum_{n=0}^{\infty} \mathrm{E}_{t-1}\left[\boldsymbol{T}_{t}^{(k)}\right] \mathbb{P}\left(\boldsymbol{n}_{t}=n\right) \\
& =\mathrm{E}_{t-1}\left[\boldsymbol{T}_{t}^{(k)}\right] .
\end{aligned}
$$

\section{B.5 Demonstração do Teorema 21}

Vamos usar o resultado que relaciona a esperança de uma variável aleatória não negativa com a sua distribuição acumulada ${ }^{2}$ [44]. Da Proposição 15, sabemos que a distribuição de $\boldsymbol{T}_{t}^{(k)} \mid \boldsymbol{P}_{t-1}, \Delta_{t-1}$ é dada por

$$
\mathbb{P}\left(\boldsymbol{T}_{t}^{(k)} \leq x \mid \boldsymbol{P}_{t-1}, \Delta_{t-1}\right)= \begin{cases}1, & x>b \boldsymbol{P}_{t-1} \\ \frac{\Psi\left(\frac{x}{P_{t-1} \Delta_{t-1}}\right)}{\Psi\left(\frac{b}{\Delta_{t-1}}\right)}, & x \leq b \boldsymbol{P}_{t-1}\end{cases}
$$

\footnotetext{
${ }^{1} \mathrm{E}\{\mathrm{E}[\boldsymbol{X} \mid \boldsymbol{Y}]\}=\mathrm{E}[\boldsymbol{X}]$.

2"'Se $\boldsymbol{X}$ [uma VA] tomar somente valores não negativos, ou seja, $\boldsymbol{X}(\omega) \geq 0 \forall \omega \in \Omega$, então $F_{X}(x)=0$ para $x<0 \mathrm{e}$

$$
\mathrm{E}(\boldsymbol{X})=\int_{0}^{\infty}\left[1-F_{X}(x)\right] d x=\int_{0}^{\infty} \mathbb{P}(\boldsymbol{X}>x) d x .
$$
}


Lembrando que, pelo Lema 20, $\mathrm{E}_{t-1}\left[\boldsymbol{P}_{t}\right]=\mathrm{E}_{t-1}\left[\boldsymbol{T}_{t}^{(k)}\right]$, temos que

$$
\begin{aligned}
\mathrm{E}_{t-1}\left[\boldsymbol{P}_{t}\right] & =\int_{0}^{b \boldsymbol{P}_{t-1}}\left(1-\frac{\Psi\left(\frac{x}{\boldsymbol{P}_{t-1} \Delta_{t-1}}\right)}{\Psi\left(\frac{b}{\Delta_{t-1}}\right)}\right) d x \\
& =b \boldsymbol{P}_{t-1}-\frac{1}{\Psi\left(\frac{b}{\Delta_{t-1}}\right)} \int_{0}^{b \boldsymbol{P}_{t-1}} \Psi\left(\frac{x}{\boldsymbol{P}_{t-1} \Delta_{t-1}}\right) d x
\end{aligned}
$$

Fazendo a substituição de variável $\frac{x}{\boldsymbol{P}_{t-1}}=\xi$ em (B.5.1), temos

$$
\begin{aligned}
\mathrm{E}_{t-1}\left[\boldsymbol{P}_{t}\right] & =b \boldsymbol{P}_{t-1}-\frac{1}{\Psi\left(\frac{b}{\Delta_{t-1}}\right)} \int_{0}^{b} \Psi\left(\frac{\xi}{\Delta_{t-1}}\right) \boldsymbol{P}_{t-1} d \xi \\
& =\boldsymbol{P}_{t-1}\left[b-\frac{1}{\Psi\left(\frac{b}{\Delta_{t-1}}\right)} \int_{0}^{b} \Psi\left(\frac{\xi}{\Delta_{t-1}}\right) d \xi\right]
\end{aligned}
$$

\section{B.6 Demonstração do Lema 22}

Usando a propriedade básica da variância condicional ${ }^{3}$ [44], temos

$$
\begin{aligned}
\operatorname{Var}_{t-1}\left(\boldsymbol{P}_{t}\right) & =\mathrm{E}_{t-1}\left[\operatorname{Var}_{t-1}\left(\boldsymbol{P}_{t} \mid \boldsymbol{n}_{t}\right)\right]+\operatorname{Var}_{t-1}\left(\mathrm{E}_{t-1}\left[\boldsymbol{P}_{t} \mid \boldsymbol{n}_{t}\right]\right) \\
& =\mathrm{E}_{t-1}\left[\operatorname{Var}_{t-1}\left(\boldsymbol{P}_{t} \mid \boldsymbol{n}_{t}\right)\right]+\operatorname{Var}_{t-1}\left(\mathrm{E}_{t-1}\left[\boldsymbol{T}_{t}^{(k)}\right]\right) .
\end{aligned}
$$

Como $\mathrm{E}_{t-1}\left[\boldsymbol{T}_{t}^{(k)}\right]$ é uma constante (dado o passado), então $\operatorname{Var}_{t-1}\left(\mathrm{E}_{t-1}\left[\boldsymbol{T}_{t}^{(k)}\right]\right)=0$. Então, resta-nos calcular

$$
\begin{aligned}
\operatorname{Var}_{t-1}\left(\boldsymbol{P}_{t} \mid \boldsymbol{n}_{t}\right) & =\operatorname{Var}_{t-1}\left(\frac{1}{\boldsymbol{n}_{t}} \sum_{k=1}^{\boldsymbol{n}_{t}} \boldsymbol{T}_{t}^{(k)} \mid \boldsymbol{n}_{t}\right) \\
& =\frac{1}{\boldsymbol{n}_{t}^{2}} \operatorname{Var}_{t-1}\left(\sum_{k=1}^{\boldsymbol{n}_{t}} \boldsymbol{T}_{t}^{(k)} \mid \boldsymbol{n}_{t}\right)
\end{aligned}
$$

Mas, pela Hipótese 4, sabemos que as variáveis $\boldsymbol{T}_{t}^{(k)}, k=1, \ldots, \boldsymbol{n}_{t}$ são condicionalmente independentes dado o passado. Portanto, a equação (B.6.2) se reduz a

$$
\begin{aligned}
\operatorname{Var}_{t-1}\left(\boldsymbol{P}_{t} \mid \boldsymbol{n}_{t}\right) & =\frac{1}{\boldsymbol{n}_{t}^{2}} \boldsymbol{n}_{t} \operatorname{Var}_{t-1}\left(\boldsymbol{T}_{t}^{(k)} \mid \boldsymbol{n}_{t}\right) \\
& =\frac{1}{\boldsymbol{n}_{t}} \operatorname{Var}_{t-1}\left(\boldsymbol{T}_{t}^{(k)}\right) .
\end{aligned}
$$

${ }^{3} \operatorname{Var}(\boldsymbol{Y})=\mathrm{E}[\operatorname{Var}(\boldsymbol{Y} \mid \boldsymbol{X})]+\operatorname{Var}(\mathrm{E}[\boldsymbol{Y} \mid \boldsymbol{X}])$. 
Substituindo (B.6.3) em (B.6.1), temos

$$
\begin{aligned}
\operatorname{Var}_{t-1}\left(\boldsymbol{P}_{t}\right) & =\mathrm{E}_{t-1}\left[\frac{1}{\boldsymbol{n}_{t}} \operatorname{Var}_{t-1}\left(\boldsymbol{T}_{t}^{(k)}\right)\right] \\
& =\operatorname{Var}_{t-1}\left(\boldsymbol{T}_{t}^{(k)}\right) \mathrm{E}_{t-1}\left[\frac{1}{\boldsymbol{n}_{t}}\right]
\end{aligned}
$$

\section{B.7 Demonstração do Lema 23}

Vamos mostrar que $\mathrm{E}\left[\frac{1}{\boldsymbol{n}_{t}}\right]$ tende a zero quando o número de agentes aumenta, ou seja, que

$$
\lim _{n \rightarrow \infty} \mathrm{E}_{t-1}\left[\frac{1}{\boldsymbol{n}_{t}}\right]=0
$$

com $n=V+C$ o número de agentes.

Para isso, vamos usar dois resultados apresentados em [45]:

Teorema. Seja $\boldsymbol{X}$ uma variável aleatória com suporte em $\mathbb{N}$. Então $\boldsymbol{X}+\epsilon>0$ quase certamente para todo $\epsilon>0$. Defina a transformada

$$
\mathcal{G}_{X}(z)=E\left[z^{X+\epsilon-1}\right], 0 \leq z \leq 1
$$

Então

$$
E\left[\frac{1}{\boldsymbol{X}+\epsilon}\right]=\int_{0}^{1} \mathcal{G}_{X}(z) d z
$$

Corolário. Seja $\boldsymbol{X} \sim \operatorname{Binomial~}(n, p)$. Então

$$
\mathrm{E}\left[\frac{1}{\boldsymbol{X}+1}\right]=\frac{1}{p(n+1)}\left[1-(1-p)^{n+1}\right] .
$$

Vamos agora calcular a transformada de $\boldsymbol{n}_{t} \mid \boldsymbol{P}_{t-1}, \Delta_{t-1}$ :

$$
\mathcal{G}_{n}(z)=\sum_{x=0}^{\min \{V, C\}} z^{x+\epsilon-1} \mathbb{P}_{t-1}\left(\boldsymbol{n}_{t}=x\right)
$$


Usando o Fato 13, temos

$$
\begin{gathered}
\mathcal{G}_{n}(t)=\sum_{x=0}^{\min \{V, C\}} z^{x+\epsilon-1} \mathbb{P}_{t-1}\left(\boldsymbol{N}_{t}^{(A)}=x\right)+\sum_{x=0}^{\min \{V, C\}} z^{x+\epsilon-1} \mathbb{P}_{t-1}\left(\boldsymbol{N}_{t}^{(B)}=x\right) \\
-\sum_{x=0}^{\min \{V, C\}} z^{x+\epsilon-1}\left[\mathbb{P}_{t-1}\left(\boldsymbol{N}_{t}^{(A)}=x\right) \mathbb{P}_{t-1}\left(\boldsymbol{N}_{t}^{(B)} \leq x\right)+\mathbb{P}_{t-1}\left(\boldsymbol{N}_{t}^{(B)}=x\right) \mathbb{P}_{t-1}\left(\boldsymbol{N}_{t}^{(A)} \leq x-1\right)\right] .
\end{gathered}
$$

Sem perda de generalidade, vamos considerar o caso $V \leq C$. Então, a transformada de $\boldsymbol{n}_{t} \mid \boldsymbol{P}_{t-1}, \Delta_{t-1}$ é dada por

$$
\mathcal{G}_{n}(z)=\mathcal{G}_{N^{(A)}}(z)+\overline{\mathcal{G}}_{N^{(B)}}(z)-h(z), z \in[0,1]
$$

onde

$$
\overline{\mathcal{G}}_{N^{(B)}}(z)=\sum_{x=0}^{V} z^{x+\epsilon-1} \mathbb{P}_{t-1}\left(\boldsymbol{N}_{t}^{(B)}=x\right) \leq \mathcal{G}_{N^{(B)}}(z), \forall z \in[0,1]
$$

e

$$
h(z) \geq 0, \forall z \in[0,1]
$$

Logo, encontramos um limitante para o valor de $\mathcal{G}_{n}(z)$, para todo $z \in[0,1]$, e também para a sua integral neste intervalo:

$$
\begin{aligned}
\mathcal{G}_{n}(z) \leq \mathcal{G}_{N^{(A)}}(z)+\mathcal{G}_{N^{(B)}}(z), & \forall z \in[0,1] \Rightarrow \\
& \Rightarrow \int_{0}^{1} \mathcal{G}_{n}(z) d z \leq \int_{0}^{1} \mathcal{G}_{N^{(A)}}(z) d z+\int_{0}^{1} \mathcal{G}_{N^{(A)}}(z) d z .
\end{aligned}
$$

Usando o teorema de [45], que relaciona a esperança da recíproca com a integral da sua transformada, temos que

$$
\mathrm{E}_{t-1}\left[\frac{1}{\boldsymbol{n}_{t}+1}\right] \leq \mathrm{E}_{t-1}\left[\frac{1}{\boldsymbol{N}_{t}^{(A)}+1}\right]+\mathrm{E}_{t-1}\left[\frac{1}{\boldsymbol{N}_{t}^{(B)}+1}\right] .
$$

Ainda, os Fatos 5 e 11 nos mostram que $\boldsymbol{N}_{t}^{(A)} \mid \boldsymbol{P}_{t-1}, \Delta_{t-1}$ e $\boldsymbol{N}_{t}^{(B)} \mid \boldsymbol{P}_{t-1}, \Delta_{t-1}$ são binomiais com parâmetros $\left(V, \Psi\left(\frac{b}{\Delta_{t-1}}\right)\right)$ e $\left(C, \mathfrak{g}_{t-1}\right)$. Usando o corolário de [45], temos que

$$
\mathrm{E}_{t-1}\left[\frac{1}{\boldsymbol{N}_{t}^{(A)}+1}\right]=\frac{1}{\Psi\left(\frac{b}{\Delta_{t-1}}\right)(V+1)}\left[1-\left(1-\Psi\left(\frac{b}{\Delta_{t-1}}\right)\right)^{V+1}\right]
$$

e

$$
\mathrm{E}_{t-1}\left[\frac{1}{\boldsymbol{N}_{t}^{(B)}+1}\right]=\frac{1}{\mathfrak{g}_{t-1}(C+1)}\left[1-\left(1-\mathfrak{g}_{t-1}\right)^{C+1}\right] .
$$


Assim, temos que

$$
\lim _{n \rightarrow \infty} \mathrm{E}_{t-1}\left[\frac{1}{\boldsymbol{N}_{t}^{(A)}+1}\right]=\lim _{n \rightarrow \infty} \mathrm{E}_{t-1}\left[\frac{1}{\boldsymbol{N}_{t}^{(B)}+1}\right]=0
$$

Como $\boldsymbol{n}_{t} \geq 0$ quase certamente, $\mathrm{E}_{t-1}\left[\frac{1}{\boldsymbol{n}_{t}+1}\right] \geq 0$. Como vale a desigualdade (B.7.1), e os limites superiores são dados por (B.7.2), aplicamos o Teorema do Confronto para obter

$$
\lim _{n \rightarrow \infty} E_{t-1}\left[\frac{1}{\boldsymbol{n}_{t}+1}\right]=0 .
$$

\section{B.8 Demonstração da Proposição 26}

Vamos mostrar que existe um $\delta^{(-)}$tal que, para todo $\delta<\delta^{(-)}, \mathfrak{h}_{\Psi, b}(\delta)<1$. Para isso, basta mostrar que

$$
\lim _{\delta \rightarrow 0} \mathfrak{h}_{\Psi, b}(\delta)=0
$$

pois, pela definição de limite, isso significa que, $\forall \varepsilon>0, \exists \delta^{(-)}>0$ tal que $\delta<\delta^{(-)} \Rightarrow$ $\mathfrak{h}_{\Psi, b}(\delta)<\varepsilon$; e tomando $\varepsilon=1$, a desigualdade é satisfeita.

Vamos então calcular $\lim _{\delta \rightarrow 0} \mathfrak{h}_{\Psi, b}(\delta)$. Lembramos que $\mathfrak{h}_{\Psi, b}(\delta)$ é dado por (3.2.5):

$$
\mathfrak{h}_{\Psi, b}(\delta)=b-\frac{1}{\Psi\left(\frac{b}{\delta}\right)} \int_{0}^{b} \Psi\left(\frac{\xi}{\delta}\right) d \xi
$$

Fazendo a substituição $y=\xi / \delta$ na integral acima, temos

$$
\mathfrak{h}_{\Psi, b}(\delta)=b-\frac{\delta}{\Psi\left(\frac{b}{\delta}\right)} \int_{0}^{b / \delta} \Psi(y) d y
$$

Considere agora a função

$$
\begin{aligned}
\mathfrak{h}_{\Psi, b}\left(\frac{b}{x}\right) & =b-\frac{b}{x \Psi(x)} \int_{0}^{x} \Psi(y) d y \\
& =b\left(1-\frac{1}{x \Psi(x)} \int_{0}^{x} \Psi(y) d y\right) .
\end{aligned}
$$

Vamos definir

$$
f(x):=\frac{1}{x \Psi(x)} \int_{0}^{x} \Psi(y) d y
$$


de forma que

$$
\mathfrak{h}_{\Psi, b}\left(\frac{b}{x}\right)=b[1-f(x)]
$$

Vamos agora calcular $\lim _{x \rightarrow \infty} f(x)$ :

$$
\lim _{x \rightarrow \infty} f(x)=\lim _{x \rightarrow \infty} \frac{1}{x \Psi(x)} \int_{0}^{x} \Psi(y) d y
$$

Usando a regra de L'Hôpital e o Teorema Fundamental do Cálculo, temos

$$
\begin{aligned}
\lim _{x \rightarrow \infty} f(x) & =\lim _{x \rightarrow \infty} \frac{1}{x \Psi(x)} \int_{0}^{x} \Psi(y) d y \\
& =\lim _{x \rightarrow \infty} \frac{\Psi(x)}{\Psi(x)+x \psi(x)} .
\end{aligned}
$$

Como $\Psi$ é função de densidade acumulada, $\lim _{x \rightarrow \infty} \Psi(x)=1$. Além disso, $\psi(x)$ é função densidade e, portanto, integrável. Assim, sabemos também que $\lim _{x \rightarrow \infty} x \psi(x)=0$. Com isso, (B.8.3) se reduz a

$$
\lim _{x \rightarrow \infty} f(x)=1
$$

Observe que $\lim _{\delta \rightarrow 0} \mathfrak{h}_{\Psi, b}(\delta)=\lim _{x \rightarrow \infty} \mathfrak{h}_{\Psi, b}\left(\frac{b}{x}\right)$. Finalmente, usando (B.8.2), temos que

$$
\begin{aligned}
\lim _{\delta \rightarrow 0} \mathfrak{h}_{\Psi, b}(\delta) & =\lim _{x \rightarrow \infty} \mathfrak{h}_{\Psi, b}\left(\frac{b}{x}\right) \\
& =b\left[1-\lim _{x \rightarrow \infty} f(x)\right] \\
& =0,
\end{aligned}
$$

o que conclui a prova.

\section{B.9 Demonstração da Proposição 27}

Vamos mostrar que existe um $\delta^{(+)}$tal que, para todo $\delta>\delta^{(+)}$, temos $\mathfrak{h}_{\Psi, b}(\delta)>1$. Para que isso seja verdade, é suficiente mostrar que

$$
\lim _{\delta \rightarrow \infty} \mathfrak{h}_{\Psi, b}(\delta)=L>1
$$

pois, pela definição de limite, isso significa que, $\forall \varepsilon>0, \exists \delta^{(+)}>0$ tal que $\delta>\delta^{(+)} \Rightarrow$ $\mathfrak{h}_{\Psi, b}(\delta)>L-\varepsilon$; e tomando $\varepsilon=L-1>0$, a desigualdade é satisfeita. 
Considere a função definida em (B.8.2):

$$
\mathfrak{h}_{\Psi, b}\left(\frac{b}{x}\right)=b[1-f(x)]
$$

$\operatorname{com} f(x)=\frac{1}{x \Psi(x)} \int_{0}^{x} \Psi(y) d y$

Vamos calcular $\lim _{x \rightarrow 0} f(x)$ :

$$
\lim _{x \rightarrow 0} f(x)=\lim _{x \rightarrow 0} \frac{1}{x \Psi(x)} \int_{0}^{x} \Psi(y) d y
$$

Usando a regra de L'Hôpital e o Teorema Fundamental do Cálculo, temos

$$
\begin{aligned}
\lim _{x \rightarrow 0} f(x) & =\lim _{x \rightarrow 0} \frac{1}{x \Psi(x)} \int_{0}^{x} \Psi(y) d y \\
& =\lim _{x \rightarrow 0} \frac{\Psi(x)}{\Psi(x)+x \psi(x)} \\
& =\lim _{x \rightarrow 0} \frac{1}{1+\frac{x \psi(x)}{\Psi(x)}} \\
& =\frac{1}{1+\lim _{x \rightarrow 0} \frac{x \psi(x)}{\Psi(x)}}
\end{aligned}
$$

Usando a hipótese da proposição, temos que $\lim _{x \rightarrow 0} \frac{x \psi(x)}{\Psi(x)}=c$. Assim,

$$
\lim _{x \rightarrow 0} f(x)=\frac{1}{1+c}
$$

Como $\lim _{\delta \rightarrow \infty} \mathfrak{h}_{\Psi, b}(\delta)=\lim _{x \rightarrow 0} \mathfrak{h}_{\Psi, b}\left(\frac{b}{x}\right)$, usando (B.8.2), temos que

$$
\begin{aligned}
\lim _{\delta \rightarrow \infty} \mathfrak{h}_{\Psi, b}(\delta) & =\lim _{x \rightarrow 0} \mathfrak{h}_{\Psi, b}\left(\frac{b}{x}\right) \\
& =b\left[1-\lim _{x \rightarrow 0} f(x)\right] \\
& =b\left(1-\frac{1}{1+c}\right) \\
& =b \frac{c}{1+c} .
\end{aligned}
$$


Note que, pela hipótese da proposição, $c>\frac{1}{b-1}$. Mas

$$
c>\frac{1}{b-1} \Leftrightarrow b c-c>1 \Leftrightarrow b c>1+c \Leftrightarrow b \frac{c}{1+c}>1
$$

ou seja, $\lim _{\delta \rightarrow \infty} \mathfrak{h}_{\Psi, b}(\delta)>1$.

\section{B.10 Demonstração da Proposição 31}

A condição (1) da proposição garante a existência e um $\delta^{\text {crítico }}$ tal que $\mathfrak{h}_{\Psi, b}\left(\delta^{\text {crítico }}\right)=1$ (ver Corolário 28). Resta-nos, então, mostrar que, sob a condição (2), esse $\delta^{\text {crítico }}$ é único. Para fazer isso, vamos calcular a derivada da função $\mathfrak{h}_{\Psi, b}(x)$ em relação a $x$ e verificar em que condições ela é maior que zero.

Por ser um cálculo muito longo, vamos usar o artifício de calcular primeiro a derivada da função

$$
\mathfrak{h}_{\Psi, b}\left(\frac{b}{x}\right)=b\left(1-\frac{1}{x \Psi(x)} \int_{0}^{x} \Psi(\xi) d \xi\right)
$$

Temos então

$$
\begin{aligned}
\frac{d}{d x} \mathfrak{h}_{\Psi, b}\left(\frac{b}{x}\right) & =-b\left[-(x \Psi(x))^{-2}(\Psi(x)+x \psi(x)) \int_{0}^{x} \Psi(\xi) d \xi+(x \Psi(x))^{-1} \Psi(x)\right] \\
= & -b\left[-(x \Psi(x))^{-2}(\Psi(x)+x \psi(x)) \int_{0}^{x} \Psi(\xi) d \xi+x^{-1}\right] \\
= & \frac{b}{x}\left[\frac{1}{x \Psi(x)^{2}}(\Psi(x)+x \psi(x)) \int_{0}^{x} \Psi(\xi) d \xi-1\right] .
\end{aligned}
$$

Usando a regra da cadeia, podemos agora calcular a derivada de $\mathfrak{h}_{\Psi, b}(x)$ :

$$
\begin{aligned}
\frac{d}{d x} \mathfrak{h}_{\Psi, b}(x) & =-\frac{b}{x^{2}} \frac{d \mathfrak{h}_{\Psi, b}\left(\frac{b}{x}\right)}{d x} \\
= & -\frac{b^{2}}{x^{3}}\left[\frac{1}{x \Psi(x)^{2}}(\Psi(x)+x \psi(x)) \int_{0}^{x} \Psi(\xi) d \xi-1\right] .
\end{aligned}
$$


Observe que $-b^{2} / x^{3}<0 \forall x>0$. Assim,

$$
\begin{aligned}
\frac{d}{d x} \mathfrak{h}_{\Psi, b}(x)>0 \Leftrightarrow \frac{1}{x \Psi(x)^{2}}(\Psi(x)+x \psi(x)) \int_{0}^{x} \Psi(\xi) d \xi-1<0 \\
\Leftrightarrow \frac{1}{x \Psi(x)^{2}}(\Psi(x)+x \psi(x)) \int_{0}^{x} \Psi(\xi) d \xi<1 \\
\quad \Leftrightarrow \int_{0}^{x} \Psi(\xi) d \xi<\frac{x \Psi(x)^{2}}{\Psi(x)+x \psi(x)} \\
\quad \Leftrightarrow \int_{0}^{x} \Psi(\xi) d \xi<\frac{x \Psi(x)}{1+\frac{x \psi(x)}{\Psi(x)}}
\end{aligned}
$$

Portanto, se $\int_{0}^{x} \Psi(\xi) d \xi<\frac{x \Psi(x)}{1+\frac{x \psi(x)}{\Psi(x)}} \forall x>0$, então $\mathfrak{h}_{\Psi, b}(\delta)$ é monotônica crescente para todo $\delta>0$, o que garante a unicidade de $\delta^{\text {crítico }}$.

\section{B.11 Demonstração da Proposição 34}

Para facilitar a notação, vamos definir o vetor

$$
x_{t}:=\left[\begin{array}{c}
p_{t} \\
\delta_{t}
\end{array}\right]
$$

e o mapa do sistema dinâmico

$$
x_{t}=\left[\begin{array}{l}
F_{1}\left(x_{t-1}\right) \\
F_{2}\left(x_{t-1}\right)
\end{array}\right] \text {, }
$$

com

$$
F_{1}(p, \delta)=p \mathfrak{h}_{\Psi, b}(\delta)
$$

e

$$
F_{2}(p, \delta)=\delta^{\alpha} \exp \left(\frac{\lambda}{1+\nu} \mathfrak{g}(p)-\lambda \frac{\nu}{1+\nu} \Psi\left(\frac{b}{\delta}\right)\right)
$$


A matriz jacobiana do sistema é dada por

$$
\begin{gathered}
J(p, \delta)=\left[\begin{array}{ll}
\frac{\partial F_{1}}{\partial p} & \frac{\partial F_{1}}{\partial \delta} \\
\frac{\partial F_{2}}{\partial p} & \frac{\partial F_{2}}{\partial \delta}
\end{array}\right] \\
=\left[\begin{array}{cc}
\mathfrak{h}_{\Psi, b}(\delta) & p \mathfrak{h}_{\Psi, b}^{\prime}(\delta) \\
\delta^{\alpha} e^{\frac{\lambda}{1+\nu}\left(\mathfrak{g}(p)-\nu \Psi\left(\frac{b}{\delta}\right)\right)} \mathfrak{g}^{\prime}(p) & e^{\frac{\lambda}{1+\nu} \mathfrak{g}(p)}\left(\alpha \delta^{\alpha-1} e^{-\lambda \frac{\nu}{1+\nu} \Psi\left(\frac{b}{\delta}\right)}+\delta^{\alpha} e^{-\lambda \frac{\nu}{1+\nu} \Psi\left(\frac{b}{\delta}\right)} \frac{b}{\delta^{2}} \lambda \frac{\nu}{1+\nu} \psi\left(\frac{b}{\delta}\right)\right)
\end{array}\right] \\
=\left[\begin{array}{cc}
\mathfrak{h}_{\Psi, b}(\delta) & p \mathfrak{h}_{\Psi, b}^{\prime}(\delta) \\
F_{2}(p, \delta) \mathfrak{g}^{\prime}(p) & \frac{1}{\delta} F_{2}(p, \delta)\left(\alpha+\frac{b}{\delta} \lambda \frac{\nu}{1+\nu} \psi\left(\frac{b}{\delta}\right)\right)
\end{array}\right] \cdot \quad \text { (B.11.3) }
\end{gathered}
$$

Lembrando que o ponto de equilíbrio $\left(p^{\star}, \delta^{\star}\right)$ é dado por (3.3.10) e (3.3.11), sabemos que

$$
\mathfrak{h}_{\Psi, b}\left(\delta^{\star}\right)=1
$$

$\mathrm{e}$

$$
\mathfrak{g}\left(p^{\star}\right)=\frac{(1-\alpha)(1+\nu)}{\lambda} \log \delta^{\star}+\nu \Psi\left(\frac{b}{\delta^{\star}}\right) .
$$

Vamos então calcular $F_{2}\left(p^{\star}, \delta^{\star}\right)$ substituindo (B.11.4) e (B.11.5) em (B.11.2):

$$
\begin{aligned}
F_{2}\left(p^{\star}, \delta^{\star}\right) & =\left(\delta^{\star}\right)^{\alpha} e^{\frac{\lambda}{1+\nu}\left(\frac{(1-\alpha)(1+\nu)}{\lambda} \log \delta^{\star}+\nu \Psi\left(\frac{b}{\delta^{\star}}\right)\right)} e^{-\lambda \frac{\nu}{1+\nu} \Psi\left(\frac{b}{\delta^{\star}}\right)} \\
& =\left(\delta^{\star}\right)^{\alpha} e^{(1-\alpha) \log \delta^{\star}} \\
& =\delta^{\star}
\end{aligned}
$$

A matriz jacobiana calculada em $\left(p^{\star}, \delta^{\star}\right)$ se reduz então a

$$
\begin{aligned}
J\left(p^{\star}, \delta^{\star}\right)=\left[\begin{array}{cc}
1 & p^{\star} \mathfrak{h}_{\Psi, b}^{\prime}\left(\delta^{\star}\right) \\
F_{2}\left(p^{\star}, \delta^{\star}\right) \mathfrak{g}^{\prime}\left(p^{\star}\right) & \frac{1}{\delta^{\star}} F_{2}\left(p^{\star}, \delta^{\star}\right) \\
\left(\alpha+\frac{b}{\delta^{\star}} \lambda \frac{\nu}{1+\nu} \psi\left(\frac{b}{\delta^{\star}}\right)\right)
\end{array}\right] \\
=\left[\begin{array}{cc}
1 & p^{\star} \mathfrak{h}_{\Psi, b}^{\prime}\left(\delta^{\star}\right) \\
\delta^{\star} \mathfrak{g}^{\prime}\left(p^{\star}\right) & \alpha+\frac{b}{\delta^{\star}} \lambda \frac{\nu}{1+\nu} \psi\left(\frac{b}{\delta^{\star}}\right)
\end{array}\right] .
\end{aligned}
$$

Os autovalores de $J\left(p^{\star}, \delta^{\star}\right)$ são a solução da seguinte equação em $\gamma$ :

$$
\operatorname{det}\left[\gamma I-J\left(p^{\star}, \delta^{\star}\right)\right]=0
$$

com $I$ a matriz identidade. 
Então, substituindo (B.11.6) em (B.11.7), a equação característica fica

$$
\gamma^{2}-\gamma\left[\alpha+\frac{b}{\delta^{\star}} \lambda \frac{\nu}{1+\nu} \psi\left(\frac{b}{\delta^{\star}}\right)\right]+\left[\alpha+\frac{b}{\delta^{\star}} \lambda \frac{\nu}{1+\nu} \psi\left(\frac{b}{\delta^{\star}}\right)-p^{\star} \delta^{\star} \mathfrak{g}^{\prime}\left(p^{\star}\right) \mathfrak{h}_{\Psi, b}^{\prime}\left(\delta^{\star}\right)\right]=0 .
$$

Para simplificar a notação e a análise, vamos definir

$$
\Lambda:=\alpha+\frac{b}{\delta^{\star}} \lambda \frac{\nu}{1+\nu} \psi\left(\frac{b}{\delta^{\star}}\right)
$$

e

$$
\Xi:=-p^{\star} \delta^{\star} \mathfrak{g}^{\prime}\left(p^{\star}\right) \mathfrak{h}_{\Psi, b}^{\prime}\left(\delta^{\star}\right)
$$

Na nova notação, a equação característica será

$$
\gamma^{2}-\Lambda \gamma+(\Lambda+\Xi)=0
$$

que é simplesmente uma equação de segundo grau em $\gamma$, e cujas soluções são dadas por

$$
\gamma_{1}=\frac{\Lambda+\sqrt{\Lambda^{2}-4(\Lambda+\Xi)}}{2}
$$

e

$$
\gamma_{2}=\frac{\Lambda-\sqrt{\Lambda^{2}-4(\Lambda+\Xi)}}{2}
$$

\section{B.12 Demonstração da Proposição 35}

Na Proposição 34, obtivemos os autovalores do sistema, que são dados por

$$
\begin{aligned}
& \gamma_{1}=\frac{\Lambda+\sqrt{\Lambda^{2}-4(\Lambda+\Xi)}}{2}, \\
& \gamma_{2}=\frac{\Lambda-\sqrt{\Lambda^{2}-4(\Lambda+\Xi)}}{2},
\end{aligned}
$$

$\operatorname{com} \Lambda=\alpha+\frac{b}{\delta^{\star}} \lambda \frac{\nu}{1+\nu} \psi\left(\frac{b}{\delta^{\star}}\right)$ e $\Xi=-p^{\star} \delta^{\star} \mathfrak{g}^{\prime}\left(p^{\star}\right) \mathfrak{h}_{\Psi, b}^{\prime}\left(\delta^{\star}\right)$.

Note que $\Lambda>0$ para quaisquer valores dos parâmetros, pois $\psi(x)>0$. Como $\mathfrak{g}(\cdot)$ é monotônica decrescente em $p$ (pela Hipótese 9), então $\mathfrak{g}^{\prime}\left(p^{\star}\right)<0$ qualquer que seja o ponto de equilíbrio. Além disso, sob a condição (2) da Proposição 31, também sabemos que $\mathfrak{h}_{\Psi, b}^{\prime}(\delta)>0$ para todo $\delta>0$. Assim, $\Xi>0$ qualquer que seja o ponto de equilíbrio. 
Os autovalores serão reais se, e somente se,

$$
\begin{aligned}
\Lambda^{2}-4(\Lambda+ & \Xi) \geq 0 \\
& \Leftrightarrow \Lambda^{2}-4 \Lambda-4 \Xi \geq 0 .
\end{aligned}
$$

Observe agora a Figura B.12.1, que representa a função $f(\Lambda)=\Lambda^{2}-4 \Lambda-4 \Xi$. Como $\Lambda>0$ e $\Xi>0$, dado um valor de $\Xi$, esta função é maior ou igual a zero se, e somente se, $\Lambda \geq 2+2 \sqrt{1+\Xi}$.

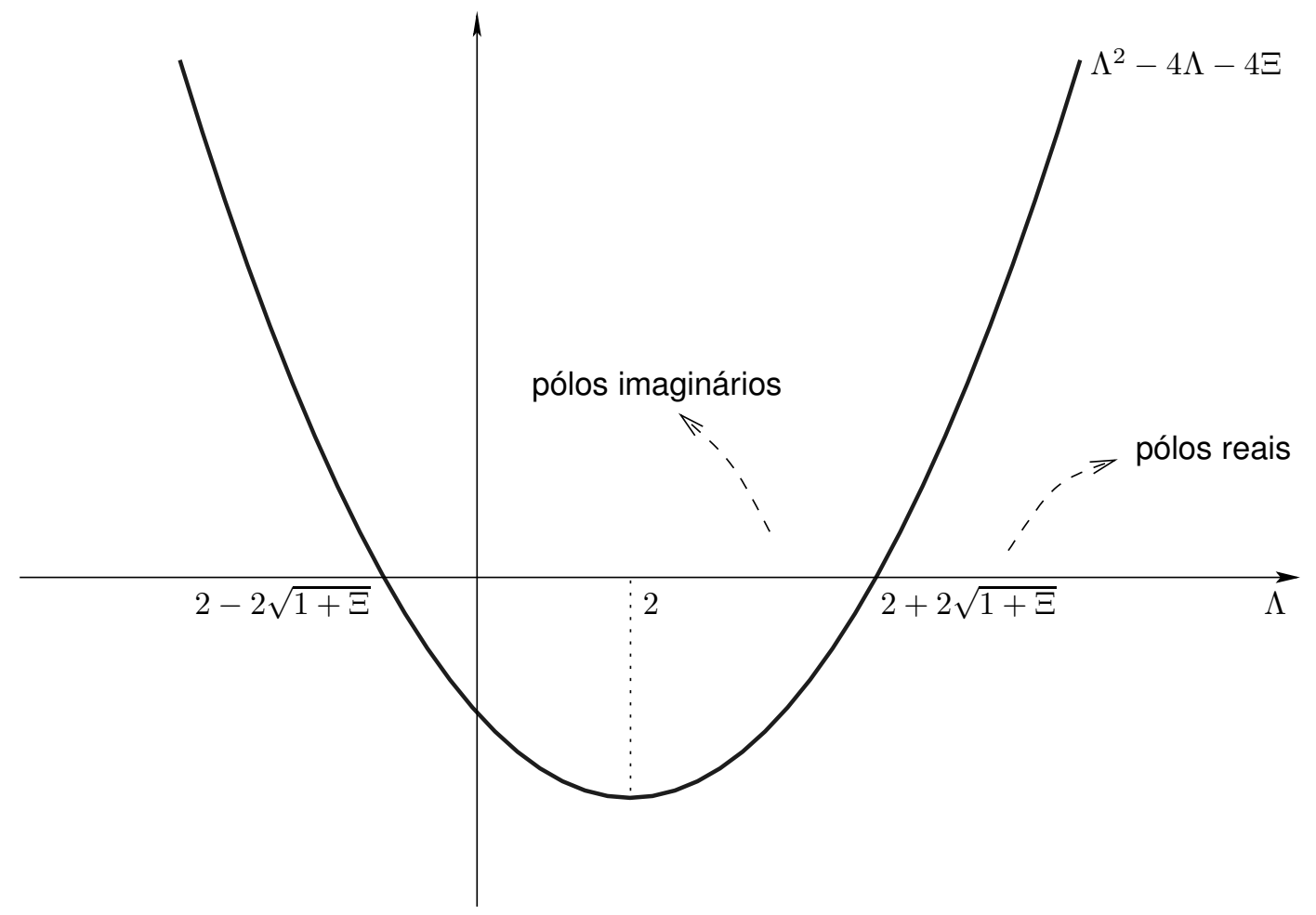

Figura B.12.1: Gráfico da função $f(\Lambda)=\Lambda^{2}-4 \Lambda-4 \Xi$, para $\Xi>0$ fixo.

Portanto,

$$
\Lambda^{2}-4(\Lambda+\Xi) \geq 0 \Leftrightarrow \Lambda \geq 2+2 \sqrt{1+\Xi}
$$

Então, sabemos que

$$
\sqrt{\Lambda^{2}-4(\Lambda+\Xi)} \in \mathbb{R} \Leftrightarrow \Lambda \geq 2+2 \sqrt{1+\Xi}
$$

ou seja,

$$
\gamma_{i} \in \mathbb{R} \Leftrightarrow \Lambda \geq 2+2 \sqrt{1+\Xi}, i=1,2
$$

Para mostrar que os autovalores serem reais implica instabilidade, observe que

$$
\Lambda \geq 2+2 \sqrt{1+\Xi} \Rightarrow \Lambda \geq 4+\epsilon_{1}, \Lambda^{2} \geq 16+\epsilon_{2},
$$


$\operatorname{com} \epsilon_{2} \geq \epsilon_{1} \geq 0$. Assim,

$$
\begin{aligned}
\gamma_{i} & \in \mathbb{R} \Leftrightarrow \Lambda \geq 2+2 \sqrt{1+\Xi} \\
& \Rightarrow \Lambda \geq 4+\epsilon_{1}, \Lambda^{2} \geq 16+\epsilon_{2} \\
& \Rightarrow \gamma_{1} \geq \frac{4+\sqrt{16}}{2} \Rightarrow \gamma_{1} \geq 4 .
\end{aligned}
$$

Como o ponto de equilíbrio só é estável se todos os autovalores tiverem módulo menor que 1 [35], quando os autovalores são reais o sistema é instável.

\section{B.13 Demonstração da Proposição 36}

A Proposição 35 nos dá uma condição necessária para que o ponto de equilíbrio $\left(p^{\star}, \delta^{\star}\right)$ seja estável: que os autovalores sejam complexos conjugados. Mas sabemos que o sistema só é estável se os autovalores tiverem módulo menor que 1.

Quando os autovalores são complexos conjugados, seu módulo é dado por

$$
\begin{aligned}
\left|\gamma_{i}\right| & =\sqrt{\frac{\Lambda^{2}}{4}+\frac{4(\Lambda+\Xi)-\Lambda^{2}}{4}} \\
& =\frac{1}{2} \sqrt{\Lambda^{2}+4 \Lambda+4 \Xi-\Lambda^{2}} \\
& =\frac{1}{2} \sqrt{4(\Lambda+\Xi)} \\
& =\sqrt{\Lambda+\Xi} .
\end{aligned}
$$

Portanto, o ponto $\left(p^{\star}, \delta^{\star}\right)$ é estável apenas quando $\Lambda+\Xi<1$. 